\title{
Iron(II) Complexes of Dimethyltriazacyclophane
}

\author{
Wei-Tsung Less \\ Loyola University Chicago, wlee5@luc.edu \\ Matthias Zeller \\ Purdue University \\ David Upp \\ Loyola University Chicago \\ Yuliya Politanska \\ Loyola University Chicago \\ Doug Steinman \\ Loyola University Chicago
}

See next page for additional authors

Follow this and additional works at: https://ecommons.luc.edu/chemistry_facpubs

Part of the Chemistry Commons

\section{Recommended Citation}

Less, Wei-Tsung; Zeller, Matthias; Upp, David; Politanska, Yuliya; Steinman, Doug; Al-Assil, Talal; and Becker, Daniel P. Ph.D.. Iron(II) Complexes of Dimethyltriazacyclophane. Acta Crystallographica Section C: Structural Chemistry, C, 74: 1641-1649, 2018. Retrieved from Loyola eCommons, Chemistry: Faculty Publications and Other Works, http://dx.doi.org/10.1107/S2053229618015255

This Article is brought to you for free and open access by the Faculty Publications and Other Works by Department at Loyola eCommons. It has been accepted for inclusion in Chemistry: Faculty Publications and Other Works by an authorized administrator of Loyola eCommons. For more information, please contact ecommons@luc.edu. c) (i) $\Theta$

This work is licensed under a Creative Commons Attribution-Noncommercial-No Derivative Works 3.0 License. (c) International Union of Crystallography 2018 
Authors

Wei-Tsung Less, Matthias Zeller, David Upp, Yuliya Politanska, Doug Steinman, Talal Al-Assil, and Daniel P. Becker Ph.D. 
STRUCTURAL

CHEMISTRY

ISSN 2053-2296

Received 1 August 2018

Accepted 29 October 2018

Edited by B. D. Santarsiero, University of Illinois at Chicago, USA

Keywords: benzo-fused TACN; diiron complex; ipso interaction; DFT calculations; azacyclophane; crystal structure; computational chemistry.

CCDC references: $1875771 ; 1875770$

Supporting information: this article has supporting information at journals.iucr.org/c

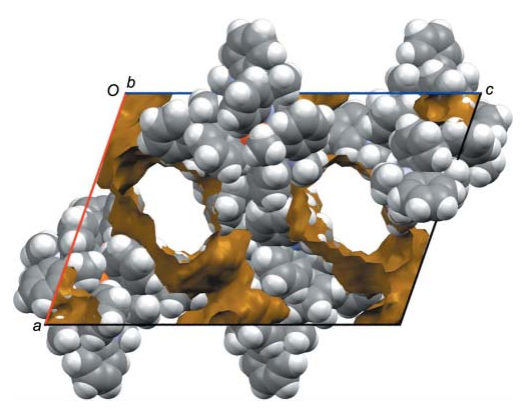

(C) 2018 International Union of Crystallography

\section{Iron(II) complexes of dimethyltriazacyclophane}

\author{
Wei-Tsung Lee, ${ }^{\mathrm{a} *}$ Matthias Zeller, ${ }^{\mathrm{b}}$ David Upp, ${ }^{\mathrm{a}}$ Yuliya Politanska, ${ }^{\mathrm{a}}$ Doug \\ Steinman, ${ }^{\mathrm{a}}$ Talal Al-Assil ${ }^{\mathrm{a}}$ and Daniel P. Becker ${ }^{\mathrm{a} *}$
}

\begin{abstract}
${ }^{a}$ Department of Chemistry and Biochemistry, Loyola University Chicago, 1032 West Sheridan Road, Chicago, Illinois 60660, USA, and ${ }^{\mathbf{b}}$ Department of Chemistry, Purdue University, 560 Oval Dr., W. Lafayette, IN 47907-2084, USA. *Correspondence e-mail: wlee5@luc.edu, dbecke3@luc.edu
\end{abstract}

Treatment of the ortho-triazacyclophane 1,4-dimethyltribenzo[ $b, e, h][1,4,7]$ triazacyclonona-2,5,8-triene $\left[\left(\mathrm{C}_{6} \mathrm{H}_{5}\right)_{3}(\mathrm{NH})\left(\mathrm{NCH}_{3}\right)_{2}, \quad\right.$ L1 $]$ with $\mathrm{Fe}\left[\mathrm{N}\left(\mathrm{SiMe}_{3}\right)_{2}\right]_{2}$ yields the dimeric iron(II) complex bis( $\mu$-1,4-dimethyltribenzo[ $b, e, h][1,4,7]$ triazacyclonona-2,5,8-trien-7-ido)bis[( $\mu$-1,4-dimethyltribenzo $[b, e, h][1,4,7]$ triazacyclonona-2,5,8-trien-7-ido)iron(II)], $\left[\mathrm{Fe}\left(\mathrm{C}_{20} \mathrm{H}_{18} \mathrm{~N}_{3}\right)_{4}\right]$ or $\mathrm{Fe}_{2}(\mathbf{L 1})_{4}(\mathbf{9})$. Dissolution of 9 in tetrahydrofuran (THF) results in solvation by two THF ligands and the formation of a simpler monoiron complex, namely bis $(\mu-1,4$ dimethyltribenzo $[b, e, h][1,4,7]$ triazacyclonona-2,5,8-trien-7-ido- $\left.\kappa N^{7}\right)$ bis(tetrahydrofuran- $\kappa O$ )iron(II), $\left[\mathrm{Fe}\left(\mathrm{C}_{20} \mathrm{H}_{18} \mathrm{~N}_{3}\right)_{2}\left(\mathrm{C}_{4} \mathrm{H}_{8} \mathrm{O}\right)_{2}\right]$ or $(\mathbf{L 1})_{2} \mathrm{Fe}(\mathrm{THF})_{2}(\mathbf{1 0})$. The reaction is reversible and $\mathbf{1 0}$ reverts in vacuo to diiron complex $\mathbf{9}$. In the structures of both $\mathbf{9}$ and $\mathbf{1 0}$, the monoanionic triazacyclophane ligand $\mathbf{L 1}^{-}$is observed in only the less-symmetric saddle conformation. No bowl-shaped crown conformers are observed in the solid state, thus preventing chelating $\kappa^{3}$-coordination to the metal as had been proposed earlier based on density functional theory (DFT) calculations. Instead, the $\mathbf{L 1}^{-}$ligands are bound in either a $\eta^{2}$-chelating fashion through the amide and one amine donor (for one of the four ligands of 9), or solely through their amide $\mathrm{N}$ atoms in an even simpler monodentate $\eta^{1}$-coordination mode. Density functional calculations on dimer 9 revealed nearly full cationic charges on each $\mathrm{Fe}$ atom and no bonding interaction between the two metal centers, consistent with the relatively long $\mathrm{Fe}$... Fe distance of 2.912 (1) $\AA$ observed in the solid state.

\section{Introduction}

Amine-containing macrocyclic ligands have been widely used and play an important role in the field of coordination chemistry due to their ability to, on many occasions, form kinetically inert metal complexes. Common among them is 1,4,7-triazacyclononane (TACN, 1, Fig. 1), which is often considered to structurally and electronically resemble the $\eta^{5}$ cyclopentadienyl $(\mathrm{Cp})$ ligand in its usual facial coordination. Given the modular nature of TACN, the straightforward introduction of chemical functionality, generally through the $\mathrm{N}$ atoms, enables modulation of the ligand, affording a variety of multidentate ligands ranging from tri- to nonadentate TACN-based ligands for supporting main-group-, transition(Yang \& Zompa, 1976), lanthanide-, and actinide-metal complexes, and the coordination chemistry has been reviewed (Yang \& Zompa, 1976; Chaudhuri \& Wieghardt, 2007). Among the transition-metal compounds are several TACN complexes of iron (Turner \& Schultz, 2001; Tse et al., 2014; Mitra et al., 2014; Boeyens et al., 1985; Kindermann et al., 2016; Tsitovich et al., 2015; Sabenya et al., 2017; Nakanishi et al., 2016; Thorarinsdottir et al., 2017).

Despite its vast utility and popularity, backbone-aryl modifications of TACN, such as monobenzo-annelated deri- 
<smiles>C1CNCCNCCN1</smiles><smiles>c1ccc2c(c1)NCCNCCN2</smiles><smiles>[124In]</smiles>

1
monobenzo-TACN

2

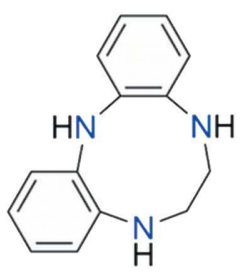

dibenzo-TACN

3<smiles>c1ccc2c(c1)Nc1ccccc1Nc1ccccc1N2</smiles>

tribenzo-TACN

4

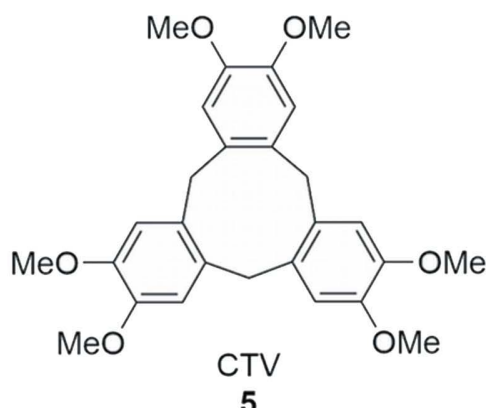

5

Figure 1

Triazacyclononane (TACN, 1) and benzo-fused TACN derivatives, including monobenzo-TACN 2, dibenzo-TACN 3, triazacyclophane 4, and cyclotriveratrylene (CTV, $\mathbf{5})$.

vative $\mathbf{2}$, dibenzoannelated derivative $\mathbf{3}$, and triazaorthocyclophane 4 (Fig. 1) remain almost completely unexplored. It is noteworthy that ancillary ligands based on aniline or phenylenediamine moieties are considered as redox noninnocent ligands and their metal complexes have drawn much attention due to unique reactivity and catalytic applications. (Chłopek et al., 2006; Lyaskovskyy \& de Bruin, 2012; Hicks, 2008; Suarez et al., 2013; Ghosh et al., 2001; Herebian et al., 2003; Van der Meer et al., 2014; Leconte et al., 2014, 2017; Kochem et al., 2013) A literature search reveals that only two examples of benzo-annelated derivatives have been prepared, (Panagopoulos et al., 2010; Samanta et al., 2012), yet no welldefined metal complexes have been successfully isolated (Samanta et al., 2012), although binding of the neutral ligand 4 to iron has been proposed and was the subject of modeling studies (Foscato et al., 2015). In contrast, several late-transition-metal complexes employing the S-donor congener, trithiaorthocyclophane, have been reported (Von Deuten et al., 1979; Von Deuten \& Klar, 1981; Kopf et al., 1979).

Cyclotriveratrylene (CTV, 5) (Collet, 1987) is a bowlshaped molecule that has been explored for applications in sensors, self-organized materials, liquid crystals, and metallosupramolecular chemistry (Hardie, 2010). CTV and its cryptophane derivatives are of great interest in molecular recognition in host-guest chemistry (Hardie, 2012). Our interest in the supramolecular scaffold CTV includes studies of the conformational dynamics of its derivatives between the bowl-shaped crown conformer and the saddle confomer (French et al., 2009), homologs of CTV (Lutz et al., 2012), and apically-modified chiral derivatives for enantio-discrimination (Lutz et al., 2018). Inspired by the conceptual combination of
CTV and TACN, we prepared 1,4-dimethyltribenzo[b,e,h]$[1,4,7]$ triazacyclonona-2,5,8-triene (7), an ortho-triazacyclophane, and 1,4,7-trimethyltribenzo[b,e,h][1,4,7]triazacyclonona2,5,8-triene (8) (Fig. 2). Specifically, we envisioned a supramolecular scaffold that could include a complexed metal, both to provide a cationic cyclophane for anion recognition, as well as to enable an electrochemically switchable host molecule. In light of the limited previous studies, we were interested in exploring the coordination chemistry of benzo-fused TACN ligands $\mathbf{7}$ and $\mathbf{8}$ with first-row transition metals, especially iron because of spin-crossover behavior, which was observed for $\left[\mathrm{Fe}(\mathrm{TACN})_{2}\right]^{2+}$ (Turner \& Schultz, 2001).

\section{Experimental}

\subsection{Material and methods}

All manipulations were performed under a nitrogen atmosphere using standard Schlenk techniques or in an $\mathrm{M}$. Braun UNIlab Pro glove-box. Glassware was dried at $150{ }^{\circ} \mathrm{C}$ overnight. Diethyl ether, $n$-pentane, tetrahydrofuran, and toluene were purified using a Pure Process Technology solvent purification system. Deuterated benzene was first dried with $\mathrm{CaH}_{2}$, then over $\mathrm{Na}$ /benzophenone, and then vacuum transferred into a storage container. Before use, an aliquot of each solvent was tested with a drop of sodium benzophenone ketyl in THF solution. All reagents were purchased from commercial vendors and used as received. $\mathrm{Fe}\left[\mathrm{N}\left(\mathrm{SiMe}_{3}\right)_{2}\right]_{2}$ was prepared according to a literature procedure (Ohki et al., 2010). ${ }^{1} \mathrm{H}$ NMR data were recorded on a Varian Inova 300 or $500 \mathrm{MHz}$ spectrometer at $22{ }^{\circ} \mathrm{C}$. Resonances in the ${ }^{1} \mathrm{H}$ NMR
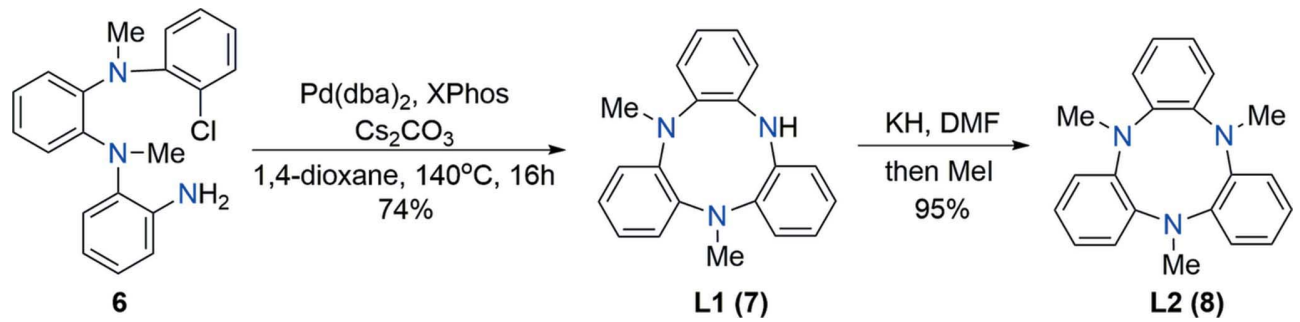

Figure 2

Optimized Buchwald-Hartwig macrocyclization of chloroaniline derivative 6 to yield $\mathbf{L 1}$ (7) and the previously reported methylation (Panagopoulos $e t$ al., 2010) to afford $\mathbf{L 2}(\mathbf{8})$. 
Table 1

Experimental details.

Crystal data

Chemical formula

$M_{\mathrm{r}}$

Crystal system, space group

Temperature (K)

$a, b, c(\AA)$

$\alpha, \beta, \gamma\left({ }^{\circ}\right)$

$V\left(\AA^{3}\right)$

$Z$

Radiation type

$\mu\left(\mathrm{mm}^{-1}\right)$

Crystal size (mm)

Data collection

Diffractometer

Absorption correction

$T_{\min }, T_{\max }$

No. of measured, independent and observed

$[I>2 \sigma(I)]$ reflections

$R_{\text {int }}$

$(\sin \theta / \lambda)_{\max }\left(\AA^{-1}\right)$

Refinement

$R\left[F^{2}>2 \sigma\left(F^{2}\right)\right], w R\left(F^{2}\right), S$

No. of reflections

No. of parameters

No. of restraints

$\mathrm{H}$-atom treatment

$\Delta \rho_{\max }, \Delta \rho_{\min }\left(\mathrm{e} \AA^{-3}\right)$

Computer programs: APEX2 (Bruker, 2014), SAINT (Bruker, 2014), SHELXS97 (Sheldrick, 2008), SHELXL2014 (Sheldrick, 2015), shelXle (Hübschle et al., 2011) and publCIF (Westrip, 2010).

spectra are referenced to residual $\mathrm{C}_{6} \mathrm{D}_{5} \mathrm{H}$ at $d=7.16$ or $\mathrm{C}_{4} \mathrm{D}_{7} \mathrm{HO}$ at $d=3.58 \mathrm{ppm}$. Solution magnetic susceptibilities were determined by the Evans method (Schubert, 1992). Elemental analysis was conducted by Midwest Microlab, LLC (Indianapolis, IN).

\subsection{Computational studies}

The CIF file of 9 was opened with SPARTAN'16 (Wavefunction, 2016). The positions of the heavy atoms were frozen, and 'Molecular Mechanics' was performed to place the $\mathrm{H}$ atoms. The number of unpaired electrons was set at 8 , and a single-point ground-state energy in the gas phase was calculated with Hartree-Fock (3-21G* basis set), using the options CONVERGE, SCFTOLERANCE=LOW, and SCFCYCLES= 900. After successful completion, this result was used as the starting point for a density functional calculation using the EDF2 method and the $6-31 G^{*}$ basis set, keeping the same options as above (CONVERGE, SCFTOLERANCE=LOW, and SCFCYCLES=900). After successful completion, this result was used as the starting point for a density functional calculation using the EDF2 method and the 6-31G* basis set with options CONVERGE and SCFCYCLES=900 but eliminating the low SCF tolerance option. After successful completion, this result was used to calculate orbitals and energies, as well as charges and bond orders.

The result of the density functional EDF2 method with the $6-31 G^{*}$ basis set was used as the starting point to calculate the single-point energy using the density functional level of theory and the B3LYP method with the $6-31 \mathrm{G}^{*}$ basis set, using the option MEM_STATIC $=502$. Continuing from this as the starting point for the wB97X-D method with the double-basis set $6-311+\mathrm{G}(3 \mathrm{df}, 2 \mathrm{p})\left[6-311 \mathrm{G}^{*}\right]$ afforded atomic charges of all atoms, including +0.961 for $\mathrm{Fe} 1$ and +0.998 for $\mathrm{Fe} 2$, as well as all HOMO/LUMO and spin-surface maps.

For direct comparison of the crystal structure with the equilibrium geometry calculated structure, the density functional $\left(6-31 \mathrm{G}^{*}\right)$ result with frozen heavy atoms was thawed (heavy atoms) and recalculated with the density functional level of theory, the EDF2 method, and the 6-31G* basis set, with COMVERGE SCFCYCLES=900, to yield the geometry optimized thawed structure.

\subsection{Synthesis and crystallization}

2.3.1. Preparation of 1,4 -dimethyltribenzo $[b, \mathrm{e}, h][1,4,7]$ triazacyclonona-2,5,8-triene (L1, 7). A $125 \mathrm{ml}$ pressure flask was charged with XPhos $(260 \mathrm{mg}, 0.54 \mathrm{mmol})$ and $\mathrm{Pd}(\mathrm{dba})_{2}$ (dba is dibenzylideneacetone; $156 \mathrm{mg}, 0.271 \mathrm{mmol}$ ), and a solution of chloroaniline, 6 (Panagopoulos et al., 2010) (504 $\mathrm{mg}, 1.49 \mathrm{mmol})$, in anhydrous 1,4-dioxane $(26 \mathrm{ml})$. The resulting solution was stirred at room temperature for $15 \mathrm{~min}$ as argon was passed over the solution. Caesium carbonate (838 $\mathrm{mg}, 2.57 \mathrm{mmol}$ ) was added and the resulting suspension was purged with argon for $30 \mathrm{~min}$. The flask was sealed and heated in a $140{ }^{\circ} \mathrm{C}$ oil bath for $16 \mathrm{~h}$. The reaction mixture was 
cooled to room temperature and filtered through a pad of Celite, and the filter cake was washed with 1:1 ( $v / v)$ methanol$\mathrm{CH}_{2} \mathrm{Cl}_{2}$. The filtrate was concentrated to dryness, leaving a brown solid which was partitioned between ethyl acetate and water. The aqueous layer was then extracted once with ethyl acetate and the organic extracts were combined, dried over $\mathrm{MgSO}_{4}$, filtered, and concentrated to dryness. The residue was dissolved in ethyl acetate and to this solution was added $6.6 \mathrm{~g}$ of Celite, and the suspension concentrated to dryness, leaving an off-white powder. This powder was then applied to the top of an $80 \mathrm{~g}$ column (Silica gel 60A, 40-75 $\mu \mathrm{m}, 200 \times 400 \mathrm{mesh}$ ) and eluted at a $42 \%$ maximum pump rate using a gradient starting with $20 \% \mathrm{CH}_{2} \mathrm{Cl}_{2}$ /petroleum ether and increasing to $30 \% \mathrm{CH}_{2} \mathrm{Cl}_{2} /$ petroleum ether, collecting $28 \mathrm{ml}$ fractions to give macrocycle 7 (L1) as an off-white powder (yield: $338 \mathrm{mg}$, 75\%; m.p. $228-230{ }^{\circ} \mathrm{C}$ ) and with ${ }^{1} \mathrm{H}$ and ${ }^{13} \mathrm{C}$ spectra identical to those reported previously (Panagopoulos et al., 2010).

2.3.2. Preparation of $\left[(\mathrm{L} 1)_{4} \mathrm{Fe}_{2}\right](9)$. To a stirred solution of L1 (394 mg, $2.0 \mathrm{mmol}$ ) in THF (4 ml) under an $\mathrm{N}_{2}$ atmosphere was added a solution of $\mathrm{Fe}\left[\mathrm{N}\left(\mathrm{SiMe}_{3}\right)_{2}\right]_{2}(377 \mathrm{mg}, 1.0 \mathrm{mmol})$ in THF $(2 \mathrm{ml})$. After $3 \mathrm{~h}$, the volatiles were removed under reduced pressure. The residue was redissolved in toluene $(5 \mathrm{ml})$ and filtered through a Celite pad. The filtrate was dried in vacuo to yield diiron complex 9 as a dark-red solid (yield: $408 \mathrm{mg}, 68 \%$ ). Crystals suitable for X-ray diffraction were grown by slow diffusion of $n$-pentane into a toluene solution at $-35{ }^{\circ} \mathrm{C}$. $\mu_{\text {eff }}=7.7(3) \mu_{\mathrm{B}}$. Analysis calculated (\%) for $\mathrm{C}_{80} \mathrm{H}_{72} \mathrm{Fe}_{2} \mathrm{~N}_{12}$ : C 73.17, H 5.53, N 12.80; found: C 73.53, H 5.35, N 12.42 .

2.3.3. Preparation of $\left[(\mathrm{L} 1)_{2} \mathrm{Fe}(\mathrm{THF})_{2}\right]$ (10). Diiron complex 9 was dissolved in THF and immediately yielded a yellowgreen solution. Crystals of monoiron complex $\mathbf{1 0}$ suitable for $\mathrm{X}$-ray diffraction were obtained by slow diffusion of $n$-pentane into a THF solution at $-35^{\circ} \mathrm{C}$. $\mu_{\text {eff }}\left(\mathrm{THF}-d_{8}\right)=5.1$ (2) $\mu_{\mathrm{B}}$. Elemental analysis for $\mathbf{1 0}$ was not obtained due to the conversion of monoiron complex $\mathbf{1 0}$ back to diiron complex $\mathbf{9}$ upon prolonged vacuum drying.

\subsection{Refinement}

If not specified otherwise, $\mathrm{H}$ atoms attached to $\mathrm{C}$ and $\mathrm{N}$ atoms were positioned geometrically and constrained to ride on their parent atoms, with $\mathrm{C}-\mathrm{H}$ bond lengths of $0.95 \AA$ for alkene and aromatic, and 0.99 and $0.98 \AA$ for aliphatic $\mathrm{CH}_{2}$ and $\mathrm{CH}_{3}$ groups, respectively. Methyl $\mathrm{H}$ atoms were allowed to rotate but not to tip to best fit the experimental electron density. $U_{\text {iso }}(\mathrm{H})$ values were set to a multiple of $U_{\text {eq }}(\mathrm{C})$, i.e. 1.5 for $\mathrm{CH}_{3}$ and 1.2 for $\mathrm{CH}_{2}$ and $\mathrm{CH}$ units, respectively.
The structure of diiron complex 9 contains solvent-accessible voids of $2479.0 \AA^{3}$ ( $c a 30 \%$ of the unit-cell volume). The residual electron-density peaks were not arranged in an interpretable pattern. The structure factors of the unresolved solvent molecules were estimated using reverse Fourier transform methods employing the SQUEEZE routine (van der Sluis \& Spek, 1990; Spek, 2015), as implemented in the program PLATON (Spek, 2009). The resultant .fab file with scattering contributions from the solvent molecules was used in the further refinement in combination with the .hkl file. The SQUEEZE procedure estimated 569.8 electrons within the solvent-accessible voids, equivalent to 11.4 molecules of toluene (the solvents of crystallization were $n$-pentane and toluene).

In monoiron complex 10, one THF ligand is disordered with two alternative orientations. The two moieties were restrained to have similar geometries, and the $U^{i j}$ components of the anisotropic displacement parameters of the atoms were restrained to be similar if closer to each other than $1.7 \AA$ (the s.u. value used was $0.01 \AA^{2}$ ). Subject to these conditions, the occupancy ratio refined to 0.790 (8):0.210 (8). Reflection 002 was affected by the beamstop and was omitted from the refinement.

Crystal data, data collection and structure refinement details are summarized in Table 1.

\section{Results and discussion}

\subsection{Optimization of the synthesis of L1 (see Fig. 1)}

Our previously reported synthesis (Panagopoulos et al., 2010) of L1 utilized a microwave-assisted intramolecular ring closure to form the tribenzotriazacyclononane ring. However, that reaction gave highly variable results, leading us to pursue reaction conditions that would yield more consistent and scalable yields. The original reaction conditions were $10 \mathrm{~mol} \%$ of $\mathrm{Pd}(\mathrm{dba})_{2}, 20 \mathrm{~mol} \%$ of racemic BINAP, and $150 \mathrm{~mol} \%$ of $\mathrm{Cs}_{2} \mathrm{CO}_{3}$ in a solvent mixture of 1:1 $(v / v)$ toluene-tert-butanol at a concentration of 0.09 molar under an argon atmosphere at $250 \mathrm{~W}$ and $130^{\circ} \mathrm{C}$ for $1 \mathrm{~h}$ to give the desired product in an optimum yield of $50 \%$. We turned our attention to the use of $\mathrm{Pd}(\mathrm{OAc})_{2}$ as a catalyst, which gave more consistent results, and varied the amount of catalyst from 10 to $60 \mathrm{~mol} \%$, revealing the optimal amount of catalyst to be $c a 20 \mathrm{~mol} \%$. We explored alternatives to toluene/tert-butanol, and improved results were seen when the solvent was changed to 1,4dioxane. The concentration of the reaction with respect to the acyclic starting material was then varied from 0.05 to $1 M$, with
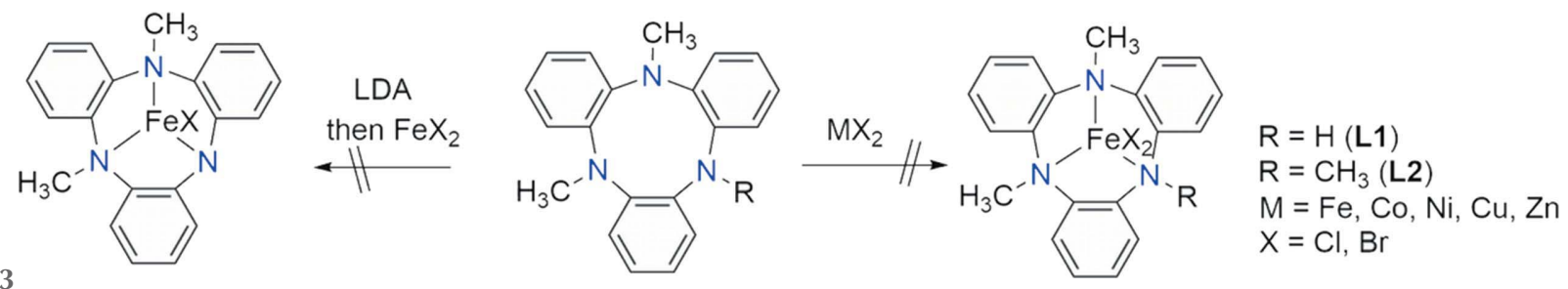

Attempted syntheses of ortho-triazacyclophane iron halide complexes. 


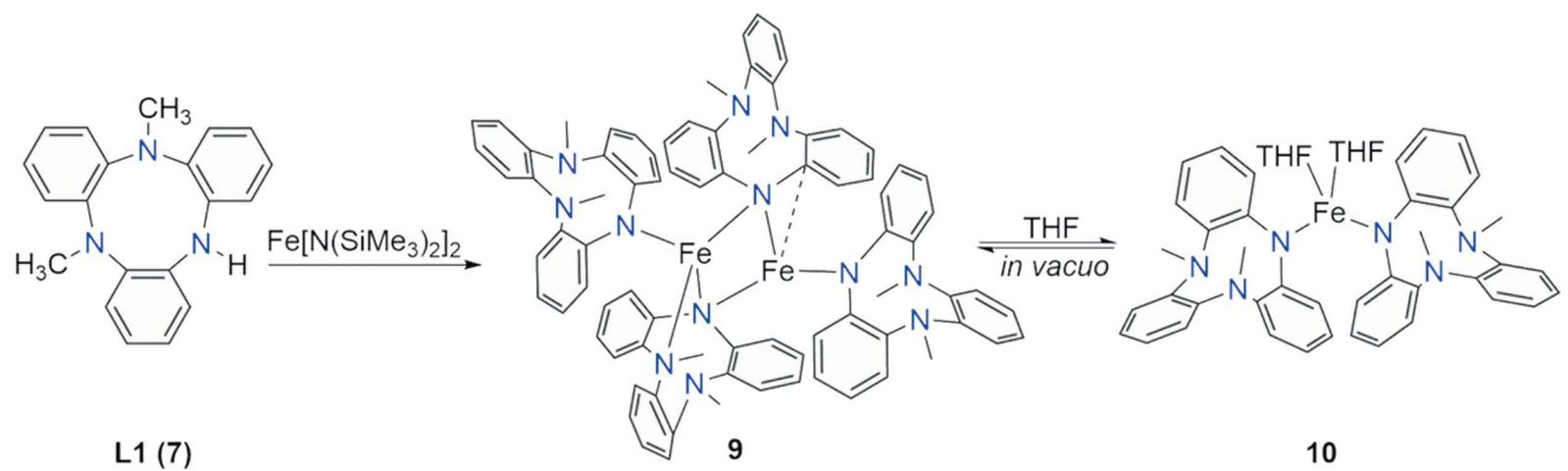

Figure 4

L1 (7)

Synthesis of diiron complex $\mathbf{9}$ and monoiron complex $\mathbf{1 0}$.

$0.05 M$ producing the best results. We next turned our attention to varying the ligand, and found that tert-Butyl XPhos gave superior results to BINAP, JohnPhos, or tri-tert-butylphosphine. Microwave heating was explored relative the conventional thermal conditions. We tried performing the reaction in the microwave on a $0.1 \mathrm{mmol}$ scale using $20 \mathrm{~mol} \%$ $\mathrm{Pd}(\mathrm{dba})_{2}, 40 \mathrm{~mol} \% \mathrm{XPhos}$, and $200 \mathrm{~mol} \% \quad \mathrm{Cs}_{2} \mathrm{CO}_{3}$ at a concentration of 0.05 molar in 1,4-dioxane at $250 \mathrm{~W}$ and $105^{\circ} \mathrm{C}$. The result was consumption of the starting material, but it required four cycles in the microwave and was not scalable. Performing the reaction on the same scale under thermal conditions in a pressure tube at $160{ }^{\circ} \mathrm{C}$ also led to consumption of the starting material, but gave a number of side products. Lowering the temperature to $140{ }^{\circ} \mathrm{C}$ for $16 \mathrm{~h}$ gave consistently higher yields of $59-74 \%$ on a scale of $2 \mathrm{mmol}$ of the acyclic amine halide, compared to yields of $50 \%$ or less on a $0.1 \mathrm{mmol}$ scale (Fig. 2). Methylation as previously

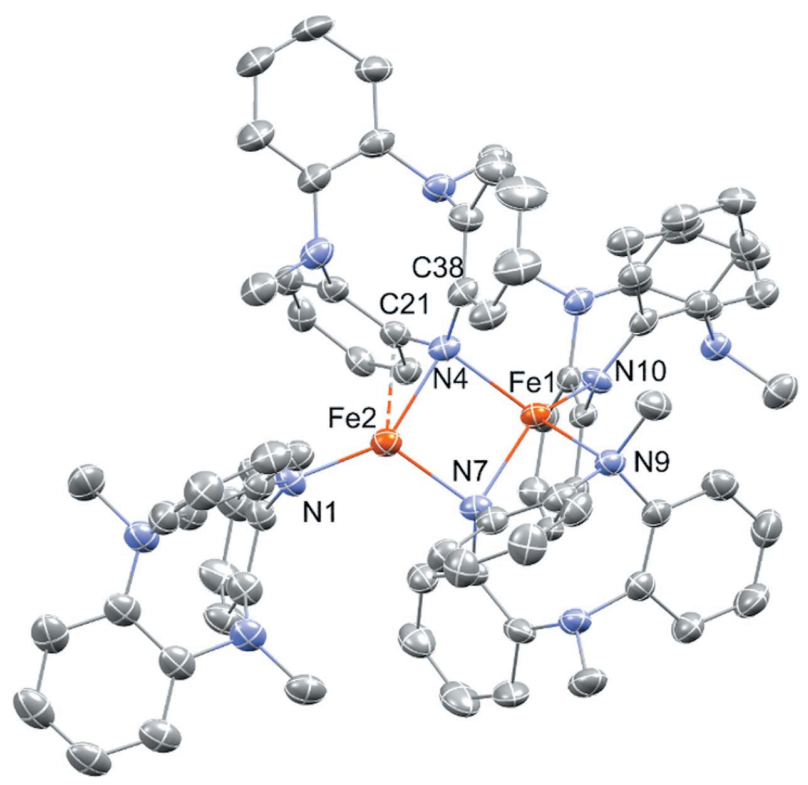

Figure 5

X-ray crystal structure of diiron complex 9. Displacement ellipsoids are shown at the $50 \%$ probability level. Color key: orange $=\mathrm{Fe}$, blue $=\mathrm{N}$, and gray $=\mathrm{C}$. $\mathrm{H}$ atoms and labels for most $\mathrm{C}$ and $\mathrm{N}$ atoms have been omitted for clarity. A displacement ellipsoid plot with $\mathrm{H}$ atoms and labels for all $\mathrm{N}$ atoms is given in the supporting information. described (Panagopoulos et al., 2010) gave the trimethyl derivative $\mathbf{L}$.

\subsection{Synthesis of iron(II) complexes}

Attempts at preparing first-row transition-metal complexes by simply mixing divalent salts of iron, cobalt, nickel, copper, or zinc with $\mathbf{L 1}$, or with $\mathbf{L 2}$, failed. A predicted molecular ion was observed when screening $\mathbf{L 1}$ and $\mathbf{L 2}$ with $\operatorname{Fe} X_{2}, \operatorname{Co} X_{2}$, $\mathrm{Ni} X_{2}(X=\mathrm{Cl}$ and $\mathrm{Br}), \mathrm{CuCl}_{2}$, or $\mathrm{ZnCl}_{2}$; however, none of these complexes could be isolated based on ${ }^{1} \mathrm{H}$ NMR spectroscopy (Fig. 3). We attribute this to the poor availability of the nitrogen lone-pair electrons due to delocalization in the aromatic rings. We therefore pursued deprotonation of $\mathbf{L 1}$, followed by transmetallation with iron halide, which was promising in producing paramagnetic products, but the mixtures were intractable. The formation of multiple species could be attributed, at least in part, to the deprotonated ligand undergoing a Smiles rearrangement (Panagopoulos et al., 2013).

Encouraged by the potential formation of inorganic complexes, an iron precursor with an internal (counter-ion) base, $\mathrm{Fe}\left[\mathrm{N}\left(\mathrm{SiMe}_{3}\right)_{2}\right]_{2}$, was used (Fig. 4). Thus, treating $\mathrm{Fe}\left[\mathrm{N}\left(\mathrm{SiMe}_{3}\right)_{2}\right]_{2}$ with $\mathbf{L 1}$ in THF afforded a yellow-green solution and, upon the removal of the volatiles, a dark-red residue was obtained. Single crystals suitable for X-ray crystallographic analysis were harvested from a saturated toluene solution stored at $-35^{\circ} \mathrm{C}$ (Table 1), and the structure was revealed to be the diiron complex 9 (Fig. 5; a depiction with complete atom labeling for $\mathrm{Fe}$ and $\mathrm{N}$ atoms is given in Fig. S1 in the supporting information). The X-ray crystal structure of 9 consists of a tetrahedral $\mathrm{Fe}^{\mathrm{II}}$ ion and a trigonal planar $\mathrm{Fe}^{\mathrm{II}}$ center bridged by two $\mathrm{Me}_{2} \mathbf{L 1}^{-}$units. Notably, 9 is a rare example of an asymmetric diiron complex. Fe1 adopts a distorted tetrahedral geometry $\left(\tau_{4}=0.69\right.$; Yang et al., 2007), coordinated by one amine, one terminal amido, and two bridging amido $\mathrm{N}$ atoms. Fe2 has a distorted trigonal-planar coordination, as quantified by the sum of the angles [359.8 (2) $)^{\circ}$ around the metal, with a narrow $\mathrm{N}-\mathrm{Fe}-\mathrm{N}$ angle of $93.0(1)^{\circ}$ between two bridging amido $\mathrm{N}$ atoms (N4 and N7). The $\mathrm{Fe}-\mathrm{N}_{\text {amido }}$ bond lengths of 1.947 (4)-2.153 (4) $\AA$ are shorter than that of $\mathrm{Fe}-\mathrm{N}_{\text {amine }}$ [2.245 (3) $\AA$ ] . Not surprisingly, the $\mathrm{Fe}-\mathrm{N}_{\text {amido }}$ bond lengths in the $\mu_{2}$-bridging amides (N4 


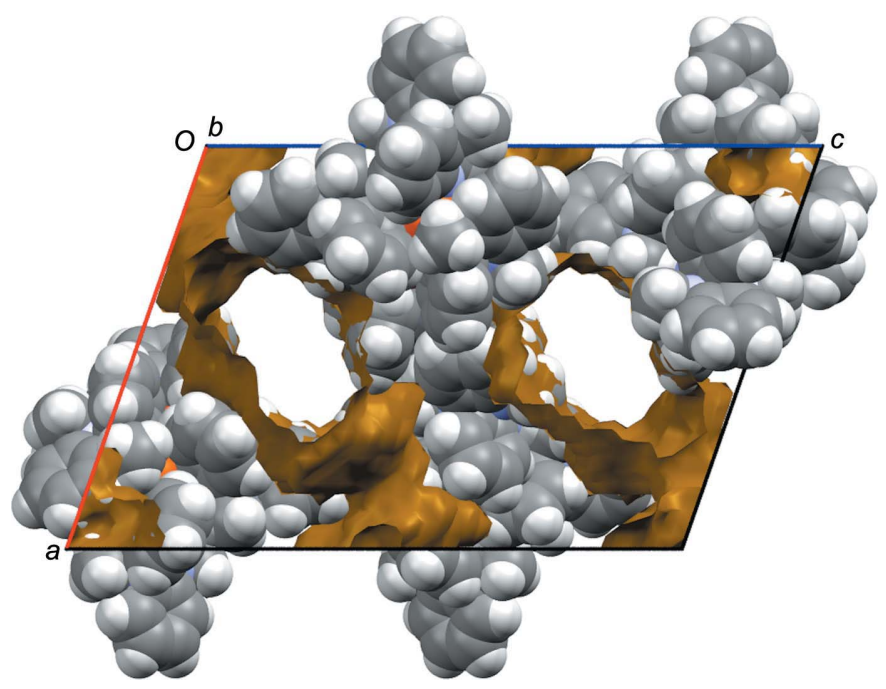

Figure 6

Solvent-accessible voids of $2479.0 \AA^{3}$ (ca $30 \%$ of the unit-cell volume) in the structure of diiron complex 9 .

and N7) are longer than those in the terminal ones (N1 and N9) (vis-à-vis, Bai et al., 2017; Deschner et al., 2011; Olmstead et al., 1991). Additional selected bond lengths and angles are given in Table 2.

In addition, the solution magnetic moment of diiron complex $9\left[7.7(3) \mu_{\mathrm{B}}\right]$ agrees well with the relatively long Fe - F Fe distance of 2.912 (1) ^ (Malassa et al., 2010; Frazier et al., 2013), which may be indicative of no interaction between the dimetal core (the covalent radii sum for $\mathrm{Fe}-\mathrm{Fe}$ is $2.48 \AA$ ).

Upon closer inspection of 9 we note that one of the $\mathbf{L 1}^{-}$ ligands coordinated to $\mathrm{Fe} 2$ is bound unsymmetrically. Besides the $\mu_{2}$-bridging amides binding, there is a stabilizing ipso interaction between one of the anilide arms and the metal center. The geometry around atom N1 is distorted tetrahedral, with the six angles ranging from $85.6(2)$ to $130.2(3)^{\circ}$. The Fe2-C21 bond length is 2.425 (4) $\AA$, more than $0.7 \AA$ shorter than the distance to the ipso carbon on the other anilide arm $[\mathrm{Fe} 2-\mathrm{C} 38=3.181$ (5) $\mathrm{A}]$. In addition, the small Fe2-N4$\mathrm{C} 21$ angle of $85.6(2)^{\circ}$ suggests the presence of a weak interaction between the $p(\pi)$ orbital on $\mathrm{C}_{i p s o}$ and the $\mathrm{Fe}^{\mathrm{II}}$ center. This ipso interaction is likely necessary to stabilize the highly electrophilic 16-electron Fe center. Such an interaction is often observed in early-transition or $f$-block metal complexes (Tonks et al., 2012; Gountchev \& Tilley, 1999; Müller-Buschbaum \& Quitmann, 2003; Odom et al., 1998; Vlaisavljevich et al., 2013; Haftbaradaran et al., 2005; Evans et al., 1996; Berthet et al., 2008; Arney et al., 1992). To the best of our knowledge, compound 9 is the first structural report on an $\mathrm{Fe}^{\mathrm{II}} \cdots \mathrm{C}_{i p s o}$ interaction (Suess \& Peters, 2013).

Significant solvent-accessible voids of $2479.0 \AA^{3}$, totalling ca $30 \%$ of the unit-cell volume, were observed in the structure of diiron complex 9 (see Fig. 6 and refinement details). One large and two smaller void areas are observed, located at the center of the unit cell, at the origin, and at the center of the $A$-face of the unit cell, with the latter two adjacent to each other. The electron density of the void area at the origin is partially
Table 2

Selected bond lengths $(\AA)$ and angles $\left(^{\circ}\right)$ for $\mathbf{9}$ and $\mathbf{1 0}$.

Atoms are labeled as indicated in Figs. 5 and 7.

\begin{tabular}{lrlrlr}
\hline \multicolumn{7}{c}{9} & \multicolumn{1}{l}{$\mathbf{1 0}$} \\
\hline $\mathrm{Fe} 1-\mathrm{N} 4$ & $2.153(4)$ & $\mathrm{N} 4-\mathrm{Fe} 1-\mathrm{N} 7$ & $89.8(1)$ & $\mathrm{Fe} 1-\mathrm{N} 1$ & $2.009(2)$ \\
$\mathrm{Fe} 1-\mathrm{N} 7$ & $2.091(3)$ & $\mathrm{N} 4-\mathrm{Fe} 1-\mathrm{N} 9$ & $122.6(1)$ & $\mathrm{Fe} 1-\mathrm{N} 4$ & $1.986(2)$ \\
$\mathrm{Fe} 1-\mathrm{N} 9$ & $1.992(4)$ & $\mathrm{N} 4-\mathrm{Fe} 1-\mathrm{N} 10$ & $115.7(1)$ & $\mathrm{Fe} 1-\mathrm{O} 1$ & $2.093(2)$ \\
$\mathrm{Fe} 1-\mathrm{N} 10$ & $2.245(3)$ & $\mathrm{N} 7-\mathrm{Fe} 1-\mathrm{N} 9$ & $79.8(1)$ & $\mathrm{Fe} 1-\mathrm{O} 2$ & $2.107(2)$ \\
& & $\mathrm{N} 7-\mathrm{Fe} 1-\mathrm{N} 10$ & $139.6(1)$ & & \\
$\mathrm{Fe} 2-\mathrm{N} 1$ & $1.947(4)$ & $\mathrm{N} 9-\mathrm{Fe} 1-\mathrm{N} 10$ & $107.5(1)$ & $\mathrm{N} 1-\mathrm{Fe} 1-\mathrm{N} 4$ & $126.64(8)$ \\
$\mathrm{Fe} 2-\mathrm{N} 4$ & $2.068(3)$ & & & $\mathrm{O} 1-\mathrm{Fe} 1-\mathrm{O} 2$ & $93.90(7)$ \\
$\mathrm{Fe} 2-\mathrm{N} 7$ & $2.061(4)$ & $\mathrm{N} 1-\mathrm{Fe} 2-\mathrm{N} 4$ & $143.6(2)$ & $\mathrm{N} 1-\mathrm{Fe} 1-\mathrm{O} 1$ & $116.48(8)$ \\
& & $\mathrm{N} 1-\mathrm{Fe} 2-\mathrm{N} 7$ & $123.2(2)$ & $\mathrm{N} 1-\mathrm{Fe} 1-\mathrm{O} 2$ & $97.67(8)$ \\
$\mathrm{Fe} 1 \cdots \mathrm{Fe} 2$ & $2.912(1)$ & $\mathrm{N} 4-\mathrm{Fe} 2-\mathrm{N} 7$ & $93.0(1)$ & $\mathrm{N} 4-\mathrm{Fe} 1-\mathrm{O} 1$ & $100.46(8)$ \\
$\mathrm{Fe} 2 \cdots \mathrm{C} 21$ & $2.425(4)$ & & & $\mathrm{N} 4-\mathrm{Fe} 1-\mathrm{O} 2$ & $117.68(8)$ \\
$\mathrm{Fe} 2 \cdots \mathrm{C} 38$ & $3.181(5)$ & & & & \\
\hline
\end{tabular}

resolved and could be interpreteted as mostly a pentane molecule disordered across an inversion center. The content of the larger and the second smaller void is highly disordered and no attempts were made to model it with disordered solvent molecules. The content of the solvent-accessible voids was estimated as ca 570 electrons, equivalent to 11.4 molecules of toluene per unit cell.

Dissolution of diiron complex 9 in THF results in solvation by two THF ligands and the formation of the simpler monoiron complex $(\mathbf{L 1})_{2} \mathrm{Fe}(\mathrm{THF})_{2}(\mathbf{1 0})$. The molecular structure and a partial numbering scheme of $\mathbf{1 0}$, as determined by X-ray crystallography, is depicted in Fig. 7. Selected bond lengths and angles are given in Table 2. The $\mathrm{C}$ atoms of one of the two coordinated THF molecules are disordered, with a refined major occupancy of 0.790 (8). Disorder and complete atom labeling are given in an additional figure in the supporting information (Fig. S2 in the supporting information). The Fe atom, unaffected by the disorder, is in a distorted tetrahedral $\left(\tau_{4}=0.82\right)$ environment, with a significantly widened $\mathrm{N}-\mathrm{Fe}-$ $\mathrm{N}$ bond angle of $126.64(8)^{\circ}$ due to steric and electrostatic repulsion between the bulky amide ions. As expected, the

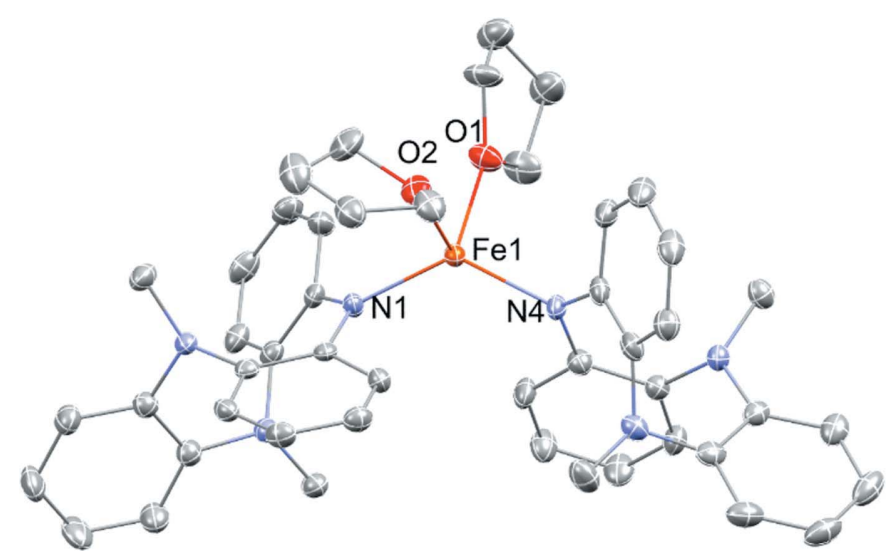

Figure 7

X-ray crystal structure of the monoiron complex 10. Displacement ellipsoids are shown at the $50 \%$ probability level. Color key: orange $=\mathrm{Fe}$, blue $=\mathrm{N}$, gray $=\mathrm{C}$, and red $=\mathrm{O}$. $\mathrm{H}$ atoms, the disorder of the THF ligands, and most labels for $\mathrm{C}$ and $\mathrm{N}$ atoms have been omitted for clarity. A fully labeled displacement ellipsoid plot with disorder shown and $\mathrm{H}$ atoms included is given in the supporting information. 


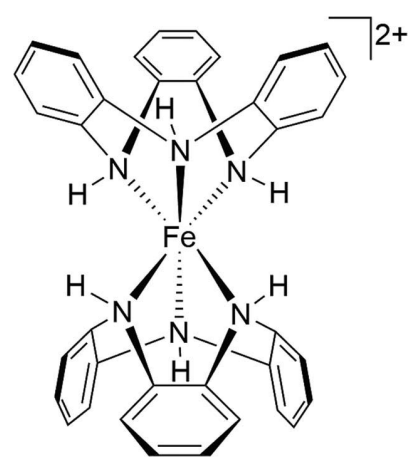

Figure 8

Calculated hypothetical structure (Foscato et al., 2015) of the dicationic $L_{2} \mathrm{Fe}$ structure, where $L=$ triazacyclophane 4 .

$\mathrm{O} 1-\mathrm{Fe} 1-\mathrm{O} 2$ angle between the two smaller ligands, $93.90(7)^{\circ}$, is less than the $\mathrm{N} 1-\mathrm{Fe} 1-\mathrm{N} 4$ angle between the two large $\mathbf{L 1}^{-}$groups. Comparable values were also observed for $\left[(\mathrm{THF})_{2} \mathrm{Fe}\left(\mathrm{SSi}^{t} \mathrm{Bu}_{3}\right)_{2}\right]$ with bulky trimethylsilyl thiolate ions. A lithium tris(diphenylamide)iron(II) complex was prepared previously by Francke \& Francke (1988).

Iron(II) $\kappa^{3}$-coordinated by unmethylated 4 (Fig. 8) has been proposed from the results of DFT calculations. We were, however, unable to obtain complexes with this binding mode. A bowl-shaped crown conformation of the ortho-triazacyclophane ligand is essential for $\kappa^{3}$-coordination, but no crown conformer was observed in either diiron complex 9 or monoiron complex 10, and only saddle conformers were observed. In 9, all the ligands are coordinated to the Fe ions through only the unmethylated (anionic) amide $\mathrm{N}$ atom, while the methyl-substituted amine donors remain unbound. In 10, one of the four ligands is coordinated in a chelating fashion involving the amide and one amine donor, but the remaining three ligands are again bound solely through their amide $\mathrm{N}$ atoms. An over-estimation in theoretical calculations for the degree of pyramidalization of the amine $\mathrm{N}$ atoms in ligands $\mathbf{L 1}$ and $\mathbf{L} 2$ could be responsible for the reluctance for this hypothetical complex to form (Foscato et al., 2015). This hypothesis is corroborated by the absence of any substantial intermolecular interactions involving the amine $\mathrm{N}$ atoms. They do not act as acceptors for any of the $\mathrm{C}-\mathrm{H} \cdots \mathrm{N}$ interactions. Indeed, intermolecular interactions in both $\mathbf{9}$ and $\mathbf{1 0}$ are sparse
Table 3

Bond orders for the $\mathrm{Fe}-\mathrm{Fe}$ and all the $\mathrm{Fe}-\mathrm{N}$ bonds in 9 .

\begin{tabular}{lllc}
\hline Entry & Atom 1 & Atom 2 & Bond order \\
\hline 1 & $\mathrm{Fe} 1$ & $\mathrm{Fe} 2$ & -0.113 \\
2 & $\mathrm{Fe} 1$ & $\mathrm{~N} 4$ & 0.342 \\
3 & $\mathrm{Fe} 1$ & $\mathrm{~N} 7$ & 0.296 \\
4 & $\mathrm{Fe} 1$ & $\mathrm{~N} 9$ & 0.253 \\
5 & $\mathrm{Fe} 1$ & $\mathrm{~N} 10$ & 0.564 \\
6 & $\mathrm{Fe} 2$ & $\mathrm{~N} 1$ & 0.556 \\
7 & $\mathrm{Fe} 2$ & $\mathrm{~N} 4$ & 0.303 \\
8 & $\mathrm{Fe} 2$ & $\mathrm{~N} 7$ & 0.383 \\
\hline
\end{tabular}

and directional interactions are limited to a number of weak $\mathrm{C}-\mathrm{H} \cdots \pi$ contacts, and shape recognition through van der Waals contacts.

\subsection{Theoretical calculations}

Bond orders in diiron complex 9 were calculated using Spartan at the density function level of theory using the EDF2 method with an $6-31 G^{*}$ basis set with heavy atoms frozen. Bond orders for $\mathrm{Fe}-\mathrm{Fe}$ and for all $\mathrm{Fe}-\mathrm{N}$ bonds are listed in Table 3. The $\mathrm{Fe}-\mathrm{Fe}$ bond order is negative, indicating no metal-metal bond, and actually some repulsion.

For direct comparison of the X-ray crystal structure of diiron complex 9 with the calculated equilibrium geometry structure, the density functional $\left(6-31 \mathrm{G}^{*}\right)$ result with frozen heavy atoms was thawed and recalculated with the density functional level of theory and the EDF2 method and the $6-31 G^{*}$ basis set to yield a geometry-optimized structure. The key bond lengths for the X-ray crystal structure of the diiron complex are summarized in Fig. 9, alongside the distances calculated for the density functional (6-31G*) optimized structure. The Fe...Fe distance of $2.912 \AA$ in the crystal structure (and further, the $\mathrm{Fe} \cdot \mathrm{Fe}$ distance in the geometryoptimized DF 6-31G* calculation of $3.012 \AA$ ) are longer than typical $\mathrm{Fe}-\mathrm{Fe}$ bonding distances of 2.46-2.78 $\AA$ (Pauling, 1976), consistent with the calculated $\mathrm{Fe}-\mathrm{Fe}$ bond order of -0.113 , indicating no metal-metal bond.

Further, a density functional calculation using the wB97X-D method with the double-basis set 6-311+G(3df,2p)[6-311G*] revealed near monocationic charges on each of the Fe atoms, as well as providing $\mathrm{HOMO} / \mathrm{LUMO}$ and spin-surface maps.

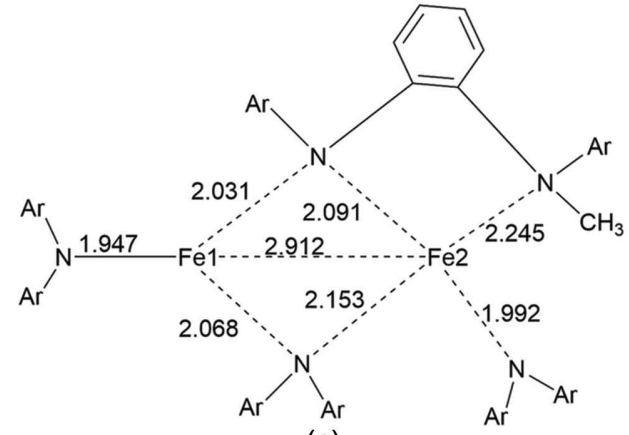

(a)

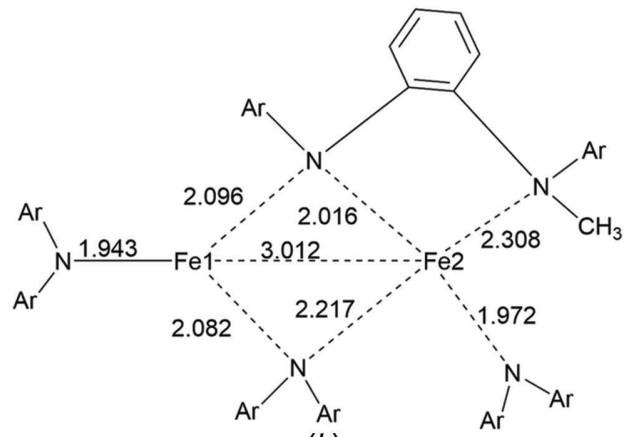

(b)

Figure 9

Key Fe bond distances in Ångströms for $(a)$ the X-ray crystal structure of diiron complex 9 and $(b)$ the density functional (6-31G*) optimized structure for 9 obtained by starting with the crystal structure and thawing the heavy atoms. 
Specifically, these calculations revealed atomic charges of +0.998 for Fe1 and +0.961 for Fe2 in diiron complex 9 .

\section{Summary}

We describe an improved Buchwald-Hartwig macrocyclization reaction for the preparation of the ortho-triazacyclophane L1, which was deprotonated by the internal $\mathrm{Fe}^{\mathrm{II}}$ base $\mathrm{Fe}\left[\mathrm{N}\left(\mathrm{SiMe}_{3}\right)_{2}\right]_{2}$ to afford the unusual diiron complex 9, as confirmed by X-ray crystallography. Diiron complex 9 was studied computationally, as well as at the density functional level of theory that, along with the longer distance between the Fe atoms, showed no evidence of metal-metal bonding. Treatment of diiron complex 9 with THF yielded the simpler $(\mathbf{L 2})_{2} \mathrm{Fe}(\mathrm{THF})_{2}$ complex 10, which reverts to diiron complex 9 in vacuo.

\section{Acknowledgements}

This work was supported by start-up funds (Loyola University Chicago). The X-ray diffractometers were funded by the National Science Foundation through the Major Research Instrumentation Program (funding for the single-crystal X-ray diffractometer).

\section{Funding information}

Funding for this research was provided by: National Science Foundation, Major Research Instrumentation (award No. DMR 1337296 to MZ).

\section{References}

Arney, D. J., Bruck, M. A., Huber, S. R. \& Wigley, D. E. (1992). Inorg. Chem. 31, 3749-3755.

Bai, T., Janes, T. \& Song, D. (2017). J. Chem. Soc. Dalton Trans. 46, $12408-12412$.

Berthet, J., Thuéry, P. \& Ephritikhine, M. (2008). Eur. J. Inorg. Chem. pp. 5455-5459.

Boeyens, J. C., Forbes, A., Hancock, R. D. \& Wieghardt, K. (1985). Inorg. Chem. 24, 2926-2931.

Bruker (2014). APEX2 and SAINT. Bruker AXS Inc., Madison, Wisconsin, USA.

Chaudhuri, P. \& Wieghardt, K. (2007). Prog. Inorg. Chem. 35, 329436.

Chłopek, K., Bothe, E., Neese, F., Weyhermüller, T. \& Wieghardt, K. (2006). Inorg. Chem. 45, 6298-6307.

Collet, A. (1987). Tetrahedron, 43, 5725-5759.

Deschner, T., Törnroos, K. W. \& Anwander, R. (2011). Inorg. Chem. 50, 7217-7228.

Evans, W. J., Ansari, M. A., Ziller, J. W. \& Khan, S. I. (1996). Inorg. Chem. 35, 5435-5444.

Foscato, M., Houghton, B. J., Occhipinti, G., Deeth, R. J. \& Jensen, V. R. (2015). J. Chem. Inf. Model. 55, 1844-1856.

Frazier, B. A., Williams, V. A., Wolczanski, P. T., Bart, S. C., Meyer, K., Cundari, T. R. \& Lobkovsky, E. B. (2013). Inorg. Chem. 52, 32953312.

French, D. C., Lutz, M. R. Jr, Lu, C., Zeller, M. \& Becker, D. P. (2009). J. Phys. Chem. A, 113, 8258-8267.

Fröhlich, H. \& Francke, H. (1988). Z. Chem. 28, 413-414.

Ghosh, A. K., Peng, S., Paul, R. L., Ward, M. D. \& Goswami, S. (2001). J. Chem. Soc. Dalton Trans. pp. 336-340.
Gountchev, T. I. \& Tilley, T. D. (1999). Organometallics, 18, 28962905.

Haftbaradaran, F., Mund, G., Batchelor, R. J., Britten, J. F. \& Leznoff, D. B. (2005). J. Chem. Soc. Dalton Trans. pp. 2343-2345.

Hardie, M. J. (2010). Chem. Soc. Rev. 39, 516-527.

Hardie, M. J. (2012). Cyclotriveratrylene and Cryptophanes, in Supramolecular Chemistry, edited by P. A. Gale \& J. W. Steed, Vol. 3, pp. 895-916. London: John Wiley \& Sons Ltd.

Herebian, D., Wieghardt, K. E. \& Neese, F. (2003). J. Am. Chem. Soc. 125, 10997-11005.

Hicks, R. G. (2008). Angew. Chem. Int. Ed. 47, 7393-7395.

Hübschle, C. B., Sheldrick, G. M. \& Dittrich, B. (2011). J. Appl. Cryst. 44, 1281-1284.

Kindermann, N., Schober, A., Demeshko, S., Lehnert, N. \& Meyer, F. (2016). Inorg. Chem. 55, 11538-11550.

Kochem, A., Gellon, G., Leconte, N., Baptiste, B., Philouze, C., Jarjayes, O., Orio, M. \& Thomas, F. (2013). Chem. Eur. J. 19, 1670716721.

Kopf, J., Von Deuten, K. \& Klar, G. (1979). Cryst. Struct. Commun. 8, 1011-1016.

Leconte, N., Ciccione, J., Gellon, G., Philouze, C. \& Thomas, F. (2014). Chem. Commun. 50, 1918-1920.

Leconte, N., Moutet, J., Herasymchuk, K., Clarke, R. M., Philouze, C., Luneau, D., Storr, T. \& Thomas, F. (2017). Chem. Commun. 53, 2764-2767.

Lutz, M. R., Ernst, E., Zeller, M., Dudzinski, J., Thoresen, P. \& Becker, D. P. (2018). Eur J. Org. Chem. doi: 10.1002ejoc.201800788.

Lutz, M. R., Zeller, M., Sarsah, S. R. S., Filipowicz, A., Wouters, H. \& Becker, D. P. (2012). Supramol. Chem. 24, 803-809.

Lyaskovskyy, V. \& de Bruin, B. (2012). ACS Catal. 2, 270-279.

Malassa, A., Agthe, C., Görls, H., Friedrich, M. \& Westerhausen, M. (2010). J. Organomet. Chem. 695, 1641-1650.

Mitra, M., Lloret-Fillol, J., Haukka, M., Costas, M. \& Nordlander, E. (2014). Chem. Commun. 50, 1408-1410.

Müller-Buschbaum, K. \& Quitmann, C. C. (2003). Z. Anorg. Allg. Chem. 629, 1610-1616.

Nakanishi, S., Kawamura, M., Sunada, Y. \& Nagashima, H. (2016). Polym. Chem. 7, 1037-1048.

Odom, A. L., Arnold, P. L. \& Cummins, C. C. (1998). J. Am. Chem. Soc. 120, 5836-5837.

Ohki, Y., Ohta, S. \& Tatsumi, K. (2010). Bio-inspired Iron and Nickel Complexes, pp. 129-147. New York: Wiley \& Sons Inc.

Olmstead, M. M., Power, P. P. \& Shoner, S. C. (1991). Inorg. Chem. 30, 2547-2551.

Panagopoulos, A. M., Steinman, D., Goncharenko, A., Geary, K., Schleisman, C., Spaargaren, E., Zeller, M. \& Becker, D. P. (2013). J. Org. Chem. 78, 3532-3540.

Panagopoulos, A. M., Zeller, M. \& Becker, D. P. (2010). J. Org. Chem. 75, 7887-7892.

Pauling, L. (1976). Proc. Natl Acad. Sci. USA, 73, 4290-4293.

Sabenya, G., Lazaro, L., Gamba, I., Martin-Diaconescu, V., Andris, E., Weyhermüller, T., Neese, F., Roithova, J., Bill, E. \& LloretFillol, J. (2017). J. Am. Chem. Soc. 139, 9168-9177.

Samanta, K., Srivastava, N., Saha, S. \& Panda, G. (2012). Org. Biomol. Chem. 10, 1553-1564.

Schubert, E. M. (1992). J. Chem. Educ. 69, 62.

Sheldrick, G. M. (2008). Acta Cryst. A64, 112-122.

Sheldrick, G. M. (2015). Acta Cryst. C71, 3-8.

Sluis, P. van der \& Spek, A. L. (1990). Acta Cryst. A46, 194-201.

Spek, A. L. (2009). Acta Cryst. D65, 148-155.

Spek, A. L. (2015). Acta Cryst. C71, 9-18.

Suarez, A. I. O., Lyaskovskyy, V., Reek, J. N., van der Vlugt, J. I. \& de Bruin, B. (2013). Angew. Chem. Int. Ed. 52, 12510-12529.

Suess, D. L. \& Peters, J. C. (2013). J. Am. Chem. Soc. 135, 4938-4941. Thorarinsdottir, A. E., Gaudette, A. I. \& Harris, T. D. (2017). Chem. Sci. 8, 2448-2456.

Tonks, I. A., Tofan, D., Weintrob, E. C., Agapie, T. \& Bercaw, J. E. (2012). Organometallics, 31, 1965-1974. 
Tse, C., Chow, T. W., Guo, Z., Lee, H. K., Huang, J. \& Che, C. (2014). Angew. Chem. Int. Ed. 53, 798-803.

Tsitovich, P. B., Cox, J. M., Benedict, J. B. \& Morrow, J. R. (2015). Inorg. Chem. 55, 700-716.

Turner, J. W. \& Schultz, F. A. (2001). Inorg. Chem. 40, 5296-5298.

Van der Meer, M., Rechkemmer, Y., Peremykin, I., Hohloch, S., van Slageren, J. \& Sarkar, B. (2014). Chem. Commun. 50, 1110411106.

Vlaisavljevich, B., Diaconescu, P. L., Lukens, W. L. Jr, Gagliardi, L. \& Cummins, C. C. (2013). Organometallics, 32, 1341-1352.
Von Deuten, K. \& Klar, G. (1981). Cryst. Struct. Commun. 10, 757764.

Von Deuten, K., Kopf, J. \& Klar, G. (1979). Cryst. Struct. Commun. 8 , 721-728.

Wavefunction (2016). SPARTAN'16. Wavefunction Inc., Irvine, CA, USA.

Westrip, S. P. (2010). J. Appl. Cryst. 43, 920-925.

Yang, L., Powell, D. R. \& Houser, R. P. (2007). Dalton Trans. pp. $955-$ 964.

Yang, R. \& Zompa, L. J. (1976). Inorg. Chem. 15, 1499-1502. 


\section{supporting information}

Acta Cryst. (2018). C74, 1641-1649 [https://doi.org/10.1107/S2053229618015255]

\section{Iron(II) complexes of dimethyltriazacyclophane}

\section{Wei-Tsung Lee, Matthias Zeller, David Upp, Yuliya Politanska, Doug Steinman, Talal Al-Assil and Daniel P. Becker}

Computing details

For both structures, data collection: APEX2 (Bruker, 2014); cell refinement: SAINT (Bruker, 2014); data reduction: SAINT (Bruker, 2014); program(s) used to solve structure: SHELXS97 (Sheldrick, 2008); program(s) used to refine structure: SHELXL2014 (Sheldrick, 2015) and SHELXLE (Hübschle et al., 2011); software used to prepare material for publication: publCIF (Westrip, 2010).

Bis $\left(\mu\right.$-1,4-dimethyltribenzo $[b, e, h][1,4,7]$ triazacyclonona-2,5,8-trien-7-ido)-1:2 $\kappa^{2} N^{1}, N^{7}: \kappa N^{7} ; 1: 2 \kappa N^{7}: \kappa^{2} N^{7}, C^{6}$ bis $\left[\left(\mu\right.\right.$-1,4-dimethyltribenzo[b,e,h][1,4,7]triazacyclonona-2,5,8-trien-7-ido- $\left.\kappa N^{7}\right)$ iron(II)] (9)

Crystal data

$\left[\mathrm{Fe}\left(\mathrm{C}_{20} \mathrm{H}_{18} \mathrm{~N}_{3}\right)_{4}\right]$

$M_{r}=1313.19$

Monoclinic, $P 2_{1} / c$

$a=21.758(2) \AA$

$b=12.7682(13) \AA$

$c=31.382(3) \AA$

$\beta=109.191(5)^{\circ}$

$V=8233.7(14) \AA^{3}$

$Z=4$

\section{Data collection}

Bruker Prospector CCD diffractometer

Radiation source: I-mu-S microsource X-ray tube

Laterally graded multilayer (Goebel) mirror monochromator

$\omega$ and phi scans

Absorption correction: multi-scan

(APEX2; Bruker, 2014)

Refinement

Refinement on $F^{2}$

Least-squares matrix: full

$R\left[F^{2}>2 \sigma\left(F^{2}\right)\right]=0.076$

$w R\left(F^{2}\right)=0.193$

$S=1.03$

14191 reflections

855 parameters

0 restraints
$F(000)=2752$

$D_{\mathrm{x}}=1.059 \mathrm{Mg} \mathrm{m}^{-3}$

$\mathrm{Cu} K \alpha$ radiation, $\lambda=1.54178 \AA$

Cell parameters from 8887 reflections

$\theta=3.0-65.8^{\circ}$

$\mu=3.17 \mathrm{~mm}^{-1}$

$T=100 \mathrm{~K}$

Rod, red

$0.11 \times 0.06 \times 0.05 \mathrm{~mm}$

$T_{\min }=0.478, T_{\max }=0.753$

52565 measured reflections

14191 independent reflections

8827 reflections with $I>2 \sigma(I)$

$R_{\text {int }}=0.133$

$\theta_{\max }=66.9^{\circ}, \theta_{\min }=2.2^{\circ}$

$h=-25 \rightarrow 25$

$k=-14 \rightarrow 15$

$l=-23 \rightarrow 37$

Primary atom site location: structure-invariant direct methods

Secondary atom site location: difference Fourier map

Hydrogen site location: inferred from neighbouring sites

$\mathrm{H}$-atom parameters constrained 


$$
\begin{array}{ll}
w=1 /\left[\sigma^{2}\left(F_{\mathrm{o}}^{2}\right)+(0.0816 P)^{2}+5.4245 P\right] & \Delta \rho_{\max }=0.79 \mathrm{e} \AA^{-3} \\
\text { where } P=\left(F_{\mathrm{o}}^{2}+2 F_{\mathrm{c}}{ }^{2}\right) / 3 & \Delta \rho_{\min }=-0.54 \mathrm{e}^{-3} \\
(\Delta / \sigma)_{\max }<0.001 &
\end{array}
$$

\section{Special details}

Geometry. All esds (except the esd in the dihedral angle between two 1.s. planes) are estimated using the full covariance matrix. The cell esds are taken into account individually in the estimation of esds in distances, angles and torsion angles; correlations between esds in cell parameters are only used when they are defined by crystal symmetry. An approximate (isotropic) treatment of cell esds is used for estimating esds involving 1.s. planes.

Refinement. The structure contains solvent accessible voids of 2479.0 Ang3 (30\% of the unit cell volume). The the residual electron density peaks were not arranged in an interpretable pattern. The hkl file was thus corrected using reverse Fourier transform methods using the SQUEEZE routine (P. van der Sluis \& Spek (1990). Acta Cryst. A46, 194-201) as

\begin{tabular}{|c|c|c|c|c|}
\hline & $x$ & $y$ & $z$ & $U_{\text {iso }} * / U_{\text {eq }}$ \\
\hline $\mathrm{C} 1$ & $0.2847(2)$ & $0.3704(4)$ & $0.38404(15)$ & $0.0336(11)$ \\
\hline $\mathrm{C} 2$ & $0.2243(2)$ & $0.3887(4)$ & $0.35057(15)$ & $0.0339(11)$ \\
\hline $\mathrm{H} 2$ & 0.2097 & 0.3425 & 0.3255 & $0.041 *$ \\
\hline C3 & $0.1860(2)$ & $0.4723(4)$ & $0.35341(17)$ & 0.0418 (13) \\
\hline H3 & 0.1453 & 0.4826 & 0.3304 & $0.050 *$ \\
\hline $\mathrm{C} 4$ & 0.2056 & $0.5413(4)$ & $0.38898(18)$ & $0.0509(15)$ \\
\hline $\mathrm{H} 4$ & 0.1784 & 0.5977 & 0.3914 & $0.061 *$ \\
\hline $\mathrm{C} 5$ & $0.2668(2)$ & $0.5262(4)$ & $0.42160(17)$ & $0.0462(14)$ \\
\hline H5 & 0.2819 & 0.5747 & 0.4457 & $0.055^{*}$ \\
\hline C6 & $0.3053(2)$ & $0.4428(4)$ & $0.41940(16)$ & $0.0367(12)$ \\
\hline $\mathrm{C} 7$ & $0.4093(2)$ & $0.5186(4)$ & $0.45291(16)$ & $0.0395(12)$ \\
\hline $\mathrm{C} 8$ & $0.4367(2)$ & $0.5861(4)$ & $0.48879(17)$ & $0.0457(14)$ \\
\hline $\mathrm{H} 8$ & 0.4317 & 0.5719 & 0.5172 & $0.055^{*}$ \\
\hline C9 & $0.4713(3)$ & $0.6743(4)$ & $0.48399(19)$ & $0.0510(15)$ \\
\hline $\mathrm{H} 9$ & 0.4911 & 0.7186 & 0.5091 & $0.061 *$ \\
\hline $\mathrm{C} 10$ & $0.4768(3)$ & $0.6969(4)$ & $0.4424(2)$ & $0.0552(16)$ \\
\hline $\mathrm{H} 10$ & 0.4971 & 0.7602 & 0.4383 & $0.066^{*}$ \\
\hline $\mathrm{C} 11$ & $0.4526(2)$ & $0.6272(4)$ & $0.40623(18)$ & 0.0487 (14) \\
\hline H11 & 0.4584 & 0.6421 & 0.3781 & $0.058^{*}$ \\
\hline $\mathrm{C} 12$ & $0.4198(2)$ & $0.5352(4)$ & $0.41123(16)$ & $0.0398(12)$ \\
\hline $\mathrm{C} 13$ & $0.4224(2)$ & $0.3570(4)$ & $0.38532(15)$ & $0.0364(12)$ \\
\hline $\mathrm{C} 14$ & $0.4872(2)$ & $0.3433(4)$ & $0.39039(16)$ & $0.0413(12)$ \\
\hline H14 & 0.5136 & 0.4031 & 0.3915 & $0.050^{*}$ \\
\hline $\mathrm{C} 15$ & 0.5148 & 0.2450 & $0.39382(17)$ & 0.0494 (14) \\
\hline $\mathrm{H} 15$ & 0.5597 & 0.2367 & 0.3975 & $0.059 *$ \\
\hline $\mathrm{C} 16$ & $0.4750(2)$ & $0.1579(4)$ & $0.39176(16)$ & $0.0446(13)$ \\
\hline H16 & 0.4925 & 0.0893 & 0.3932 & $0.053^{*}$ \\
\hline $\mathrm{C} 17$ & $0.4108(2)$ & $0.1712(4)$ & $0.38768(15)$ & $0.0362(11)$ \\
\hline H17 & 0.3846 & 0.1109 & 0.3861 & $0.043^{*}$ \\
\hline $\mathrm{C} 18$ & $0.3823(2)$ & $0.2705(4)$ & $0.38573(15)$ & $0.0336(11)$ \\
\hline
\end{tabular}
implemented in the program Platon. The resultant files were used in the further refinement. (The FAB file with details of the Squeeze results is appended to this cif file). The Squeeze procedure corrected for 569.8 electrons within the solvent accesible voids, equivalent of eight molecules of tetrahydro furane (the crystallization solvent).

Fractional atomic coordinates and isotropic or equivalent isotropic displacement parameters $\left(\AA^{2}\right)$ 


\begin{tabular}{|c|c|c|c|c|}
\hline C19 & $0.3620(2)$ & 0.4097 (4) & $0.49871(16)$ & $0.0444(13)$ \\
\hline H19A & 0.3348 & 0.3473 & 0.4966 & $0.067^{*}$ \\
\hline H19B & 0.3417 & 0.4697 & 0.5083 & $0.067^{*}$ \\
\hline $\mathrm{H} 19 \mathrm{C}$ & 0.4052 & 0.3973 & 0.5208 & $0.067^{*}$ \\
\hline $\mathrm{C} 20$ & $0.3840(3)$ & $0.4952(4)$ & $0.33024(16)$ & $0.0550(15)$ \\
\hline $\mathrm{H} 20 \mathrm{~A}$ & 0.3566 & 0.4434 & 0.3095 & $0.083^{*}$ \\
\hline H20B & 0.4257 & 0.5011 & 0.3249 & $0.083^{*}$ \\
\hline $\mathrm{H} 20 \mathrm{C}$ & 0.3620 & 0.5633 & 0.3251 & $0.083^{*}$ \\
\hline $\mathrm{C} 21$ & $0.1671(2)$ & $0.1208(3)$ & $0.31601(14)$ & $0.0284(10)$ \\
\hline $\mathrm{C} 22$ & $0.1206(2)$ & $0.1744(3)$ & $0.32995(14)$ & $0.0285(10)$ \\
\hline $\mathrm{H} 22$ & 0.1141 & 0.1550 & 0.3574 & $0.034^{*}$ \\
\hline $\mathrm{C} 23$ & $0.0841(2)$ & $0.2552(3)$ & $0.30420(15)$ & $0.0304(10)$ \\
\hline $\mathrm{H} 23$ & 0.0532 & 0.2909 & 0.3143 & $0.036^{*}$ \\
\hline $\mathrm{C} 24$ & $0.0924(2)$ & $0.2842(4)$ & $0.26395(15)$ & 0.0334 \\
\hline $\mathrm{H} 24$ & 0.0662 & 0.3374 & 0.2457 & $0.040^{*}$ \\
\hline $\mathrm{C} 25$ & $0.1398(2)$ & $0.2342(4)$ & $0.25073(15)$ & $0.0320(11)$ \\
\hline $\mathrm{H} 25$ & 0.1467 & 0.2555 & 0.2237 & $0.038^{*}$ \\
\hline $\mathrm{C} 26$ & $0.1770(2)$ & 0.1544 (4) & $0.27575(14)$ & 0.0313 \\
\hline $\mathrm{C} 27$ & $0.2018(2)$ & $0.0586(4)$ & $0.21864(15)$ & $0.0380(12)$ \\
\hline $\mathrm{C} 28$ & $0.2236(3)$ & 0.0819 (4) & $0.18263(17)$ & 0.0528 \\
\hline $\mathrm{H} 28$ & 0.2593 & 0.1282 & 0.1870 & $0.063^{*}$ \\
\hline C29 & $0.1935(3)$ & $0.0383(5)$ & $0.14064(18)$ & $0.0631(18)$ \\
\hline $\mathrm{H} 29$ & 0.2095 & 0.0540 & 0.1166 & $0.076^{*}$ \\
\hline $\mathrm{C} 30$ & $0.1410(3)$ & $-0.0274(5)$ & $0.13302(17)$ & $0.0627(18)$ \\
\hline H30 & 0.1192 & -0.0539 & 0.1036 & $0.075^{*}$ \\
\hline $\mathrm{C} 31$ & $0.1202(3)$ & $-0.0548(4)$ & $0.16902(15)$ & 0.0470 \\
\hline H31 & 0.0844 & -0.1014 & 0.1641 & $0.056^{*}$ \\
\hline C32 & 0.1507 (2) & -0.0152 & $0.21207(15)$ & $0.0376(12)$ \\
\hline $\mathrm{C} 33$ & 0.1904 (2) & $-0.0967(4)$ & $0.28417(15)$ & $0.0356(11)$ \\
\hline C34 & $0.2103(3)$ & $-0.1934(4)$ & $0.27291(17)$ & $0.0436(13)$ \\
\hline H34 & 0.1873 & -0.2231 & 0.2443 & $0.052^{*}$ \\
\hline $\mathrm{C} 35$ & $0.2626(3)$ & $-0.2482(4)$ & $0.30196(19)$ & $0.0604(17)$ \\
\hline H35 & 0.2749 & -0.3147 & 0.2939 & $0.072 *$ \\
\hline C36 & $0.2962(3)$ & $-0.2022(5)$ & $0.34327(18)$ & $0.0571(16)$ \\
\hline H36 & 0.3320 & -0.2378 & 0.3638 & $0.069 *$ \\
\hline C37 & $0.2781(2)$ & -0.1059 (4) & $0.35466(15)$ & $0.0386(12)$ \\
\hline H37 & 0.3031 & -0.0749 & 0.3825 & $0.046^{*}$ \\
\hline C38 & $0.2235(2)$ & $-0.0516(4)$ & $0.32614(14)$ & $0.0301(10)$ \\
\hline C39 & $0.2869(2)$ & $0.1660(4)$ & $0.27151(16)$ & $0.0429(13)$ \\
\hline H39A & 0.3206 & 0.1233 & 0.2655 & $0.064^{*}$ \\
\hline H39B & 0.3015 & 0.1867 & 0.3034 & $0.064^{*}$ \\
\hline $\mathrm{H} 39 \mathrm{C}$ & 0.2788 & 0.2288 & 0.2525 & $0.064^{*}$ \\
\hline $\mathrm{C} 40$ & $0.0735(2)$ & $-0.0973(4)$ & $0.24384(15)$ & $0.0366(12)$ \\
\hline $\mathrm{H} 40 \mathrm{~A}$ & 0.0389 & -0.0560 & 0.2226 & $0.055^{*}$ \\
\hline $\mathrm{H} 40 \mathrm{~B}$ & 0.0650 & -0.1033 & 0.2726 & $0.055^{*}$ \\
\hline $\mathrm{H} 40 \mathrm{C}$ & 0.0745 & -0.1674 & 0.2314 & $0.055^{*}$ \\
\hline C41 & $0.2244(2)$ & 0.1888 (4) & $0.46888(15)$ & 0.0305 \\
\hline $\mathrm{C} 42$ & 0.1949 (2) & $0.2835(4)$ & $0.45118(16)$ & $0.0345(11)$ \\
\hline
\end{tabular}




\begin{tabular}{|c|c|c|c|c|}
\hline $\mathrm{H} 42$ & 0.1842 & 0.2963 & 0.4198 & $0.041^{*}$ \\
\hline $\mathrm{C} 43$ & 0.1809 & $0.3585(4)$ & $0.47771(19)$ & $0.0470(13)$ \\
\hline $\mathrm{H} 43$ & 0.1602 & 0.4217 & 0.4646 & $0.056^{*}$ \\
\hline $\mathrm{C} 44$ & 0.1968 (3) & $0.3425(4)$ & $0.5234(2)$ & 0.0585 \\
\hline $\mathrm{H} 44$ & 0.1886 & 0.3953 & 0.5422 & $0.070^{*}$ \\
\hline $\mathrm{C} 45$ & $0.2250(3)$ & $0.2480(4)$ & $0.54172(17)$ & 0.0469 (14) \\
\hline $\mathrm{H} 45$ & 0.2361 & 0.2370 & 0.5733 & $0.056^{*}$ \\
\hline $\mathrm{C} 46$ & $0.2375(2)$ & 0.1695 (4) & $0.51516(15)$ & 0.0356 \\
\hline $\mathrm{C} 47$ & $0.2139(2)$ & $-0.0121(4)$ & $0.52683(15)$ & 0.0350 \\
\hline $\mathrm{C} 48$ & $0.1787(2)$ & $-0.0102(4)$ & $0.55643(16)$ & $0.0427(12)$ \\
\hline $\mathrm{H} 48$ & 0.1885 & 0.0425 & 0.5791 & $0.051^{*}$ \\
\hline C49 & 0.1303 & $-0.0812(5)$ & $0.55451(17)$ & $0.0517(14)$ \\
\hline $\mathrm{H} 49$ & 0.1075 & -0.0789 & 0.5757 & $0.062 *$ \\
\hline $\mathrm{C} 50$ & 0.1159 (3) & -0.1555 (4) & $0.52102(17)$ & $0.0467(13)$ \\
\hline H50 & 0.0826 & -0.2054 & 0.5189 & $0.056^{*}$ \\
\hline C51 & $0.1492(2)$ & $-0.1588(4)$ & $0.49038(16)$ & $0.0427(13)$ \\
\hline H51 & 0.1375 & -0.2096 & 0.4670 & $0.051^{*}$ \\
\hline C52 & $0.1998(2)$ & $-0.0884(4)$ & $0.49323(14)$ & 0.0319 \\
\hline $\mathrm{C} 53$ & $0.2967(2)$ & $-0.0500(4)$ & $0.47170(15)$ & 0.0334 \\
\hline C54 & $0.3533(2)$ & $-0.1058(4)$ & $0.49256(15)$ & $0.0400(12)$ \\
\hline H54 & 0.3512 & -0.1785 & 0.4984 & $0.048^{*}$ \\
\hline $\mathrm{C} 55$ & $0.4128(2)$ & $-0.0553(4)$ & $0.50488(17)$ & 0.0474 \\
\hline H55 & 0.4515 & -0.0936 & 0.5192 & $0.057^{*}$ \\
\hline C56 & $0.4163(2)$ & $0.0504(4)$ & $0.49650(17)$ & 0.0459 (13) \\
\hline H56 & 0.4573 & 0.0849 & 0.5053 & $0.055^{*}$ \\
\hline $\mathrm{C} 57$ & $0.3599(2)$ & 0.1058 & $0.47530(15)$ & $0.0371(12)$ \\
\hline H57 & 0.3625 & 0.1784 & 0.4694 & $0.044 *$ \\
\hline C58 & $0.2997(2)$ & $0.0570(4)$ & $0.46250(14)$ & 0.0309 \\
\hline C59 & $0.3147(2)$ & $0.0667(4)$ & $0.57586(15)$ & 0.0479 (14) \\
\hline H59A & 0.3490 & 0.1159 & 0.5752 & $0.058^{*}$ \\
\hline H59B & 0.3329 & -0.0041 & 0.5817 & $0.058^{*}$ \\
\hline $\mathrm{H} 59 \mathrm{C}$ & 0.2973 & 0.0870 & 0.5998 & $0.058^{*}$ \\
\hline C60 & $0.2344(3)$ & $-0.2094(4)$ & $0.44436(16)$ & $0.0427(13)$ \\
\hline $\mathrm{H} 60 \mathrm{~A}$ & 0.1900 & -0.2332 & 0.4282 & $0.064^{*}$ \\
\hline H60B & 0.2536 & -0.2539 & 0.4709 & $0.064 *$ \\
\hline $\mathrm{H} 60 \mathrm{C}$ & 0.2607 & -0.2139 & 0.4244 & $0.064 *$ \\
\hline C61 & $0.0408(2)$ & $0.0432(3)$ & $0.39382(13)$ & $0.0273(10)$ \\
\hline C62 & $0.0637(2)$ & $0.1056(4)$ & $0.43271(14)$ & $0.0309(11)$ \\
\hline H62 & 0.1019 & 0.0843 & 0.4561 & $0.037 *$ \\
\hline C63 & $0.0333(2)$ & $0.1961(3)$ & $0.43842(14)$ & 0.0313 \\
\hline H63 & 0.0513 & 0.2370 & 0.4649 & $0.038^{*}$ \\
\hline C64 & $-0.0238(2)$ & $0.2280(4)$ & $0.40555(15)$ & 0.0342 \\
\hline H64 & -0.0452 & 0.2909 & 0.4088 & $0.041^{*}$ \\
\hline C65 & $-0.0486(2)$ & $0.1649(3)$ & $0.36772(14)$ & $0.0291(10)$ \\
\hline H65 & -0.0884 & 0.1846 & 0.3455 & $0.035^{*}$ \\
\hline C66 & $-0.0181(2)$ & $0.0751(3)$ & $0.36100(13)$ & $0.0257(10)$ \\
\hline C67 & $-0.1073(2)$ & $-0.0313(4)$ & $0.31871(14)$ & 0.0325 \\
\hline C68 & $-0.1663(2)$ & -0.0093 & $0.28458(15)$ & $0.0372(12)$ \\
\hline
\end{tabular}




\begin{tabular}{|c|c|c|c|c|}
\hline H68 & -0.1667 & 0.0356 & 0.2604 & $0.045^{*}$ \\
\hline C69 & $-0.2235(2)$ & $-0.0522(4)$ & $0.28602(16)$ & $0.0427(13)$ \\
\hline H69 & -0.2631 & -0.0365 & 0.2628 & $0.051^{*}$ \\
\hline $\mathrm{C} 70$ & $-0.2242(2)$ & $-0.1180(4)$ & $0.32079(16)$ & $0.0411(12)$ \\
\hline $\mathrm{H} 70$ & -0.2641 & -0.1455 & 0.3221 & $0.049^{*}$ \\
\hline C71 & $-0.1657(2)$ & $-0.1435(4)$ & $0.35389(15)$ & $0.0348(11)$ \\
\hline H71 & -0.1658 & -0.1907 & 0.3773 & $0.042 *$ \\
\hline $\mathrm{C} 72$ & $-0.1073(2)$ & $-0.1012(4)$ & $0.35337(14)$ & $0.0317(11)$ \\
\hline $\mathrm{C} 73$ & $-0.0028(2)$ & $-0.1795(4)$ & $0.36796(14)$ & $0.0320(11)$ \\
\hline C74 & $-0.0188(2)$ & $-0.2791(4)$ & $0.34968(15)$ & $0.0356(11)$ \\
\hline H74 & -0.0590 & -0.3087 & 0.3496 & $0.043 *$ \\
\hline $\mathrm{C} 75$ & $0.0201(2)$ & $-0.3376(4)$ & $0.33164(15)$ & $0.0393(12)$ \\
\hline H75 & 0.0075 & -0.4055 & 0.3195 & $0.047 *$ \\
\hline $\mathrm{C} 76$ & $0.0781(2)$ & $-0.2930(4)$ & $0.33209(14)$ & $0.0356(11)$ \\
\hline H76 & 0.1059 & -0.3306 & 0.3197 & $0.043^{*}$ \\
\hline C77 & $0.0964(2)$ & $-0.1947(4)$ & $0.35019(14)$ & $0.0325(11)$ \\
\hline H77 & 0.1368 & -0.1667 & 0.3501 & $0.039^{*}$ \\
\hline $\mathrm{C} 78$ & $0.0569(2)$ & $-0.1339(3)$ & $0.36900(13)$ & $0.0280(10)$ \\
\hline C79 & $-0.0450(2)$ & $0.0685(4)$ & $0.27994(14)$ & $0.0354(11)$ \\
\hline $\mathrm{H} 79 \mathrm{~A}$ & -0.0621 & 0.0224 & 0.2537 & $0.053^{*}$ \\
\hline H79B & 0.0001 & 0.0873 & 0.2835 & $0.053^{*}$ \\
\hline $\mathrm{H} 79 \mathrm{C}$ & -0.0715 & 0.1321 & 0.2754 & $0.053^{*}$ \\
\hline C 80 & $-0.0478(2)$ & $-0.1707(4)$ & $0.42861(15)$ & $0.0432(13)$ \\
\hline $\mathrm{H} 80 \mathrm{~A}$ & -0.0658 & -0.2417 & 0.4235 & $0.065^{*}$ \\
\hline H80B & -0.0751 & -0.1268 & 0.4408 & $0.065^{*}$ \\
\hline $\mathrm{H} 80 \mathrm{C}$ & -0.0035 & -0.1731 & 0.4502 & $0.065^{*}$ \\
\hline $\mathrm{Fe} 1$ & $0.17367(3)$ & $0.00329(6)$ & $0.40199(2)$ & $0.02762(18)$ \\
\hline $\mathrm{Fe} 2$ & $0.25881(3)$ & $0.15927(6)$ & $0.38298(2)$ & $0.03122(19)$ \\
\hline N1 & $0.31703(17)$ & $0.2743(3)$ & $0.38190(12)$ & $0.0315(9)$ \\
\hline N2 & $0.36834(18)$ & $0.4317(3)$ & $0.45443(13)$ & $0.0384(10)$ \\
\hline N3 & $0.39530(19)$ & $0.4622(3)$ & $0.37670(13)$ & $0.0399(10)$ \\
\hline N4 & $0.20378(16)$ & $0.0392(3)$ & $0.34477(11)$ & $0.0277(8)$ \\
\hline N5 & $0.22650(18)$ & $0.1048(3)$ & $0.26126(12)$ & $0.0374(10)$ \\
\hline N6 & $0.13593(18)$ & $-0.0458(3)$ & $0.25134(12)$ & $0.0330(9)$ \\
\hline N7 & 0.24007 (17) & $0.1133(3)$ & $0.44047(11)$ & $0.0292(9)$ \\
\hline N8 & $0.26247(18)$ & $0.0687(3)$ & $0.53236(12)$ & $0.0344(9)$ \\
\hline N9 & $0.23263(18)$ & $-0.0987(3)$ & $0.45908(11)$ & $0.0317(9)$ \\
\hline N10 & $0.08062(16)$ & $-0.0371(3)$ & $0.38772(11)$ & $0.0268(8)$ \\
\hline N11 & $-0.04708(17)$ & $0.0144(3)$ & $0.32030(11)$ & $0.0298(9)$ \\
\hline N12 & $-0.04644(17)$ & $-0.1268(3)$ & $0.38618(11)$ & $0.0282(8)$ \\
\hline
\end{tabular}

Atomic displacement parameters $\left(\AA^{2}\right)$

\begin{tabular}{lllllll}
\hline & $U^{11}$ & $U^{22}$ & $U^{33}$ & $U^{12}$ & $U^{13}$ & $U^{23}$ \\
\hline $\mathrm{C} 1$ & $0.028(2)$ & $0.033(3)$ & $0.035(3)$ & $-0.002(2)$ & $0.004(2)$ & $0.002(2)$ \\
$\mathrm{C} 2$ & $0.034(3)$ & $0.026(3)$ & $0.035(3)$ & $-0.001(2)$ & $0.002(2)$ & $0.001(2)$ \\
$\mathrm{C} 3$ & $0.031(3)$ & $0.034(3)$ & $0.046(3)$ & $0.003(2)$ & $-0.007(2)$ & $-0.001(2)$ \\
$\mathrm{C} 4$ & $0.039(3)$ & $0.038(3)$ & $0.061(4)$ & $0.016(2)$ & $-0.004(3)$ & $-0.006(3)$
\end{tabular}




\begin{tabular}{|c|c|c|c|c|c|c|}
\hline $\mathrm{C} 5$ & 0.040 & $0.042(3)$ & $0.046(3)$ & $0.004(2)$ & $-0.001(2)$ & -0.009 \\
\hline C6 & $0.022(2)$ & $0.040(3)$ & $0.041(3)$ & $0.005(2)$ & $0.000(2)$ & $0.003(2)$ \\
\hline $\mathrm{C} 7$ & 0.029 (3) & $0.035(3)$ & $0.041(3)$ & 0.007 (2) & $-0.007(2)$ & $0.005(2)$ \\
\hline $\mathrm{C} 8$ & $0.036(3)$ & $0.043(3)$ & $0.045(3)$ & $0.007(3)$ & $-0.004(2)$ & $-0.004(2)$ \\
\hline $\mathrm{C} 9$ & $0.039(3)$ & $0.045(3)$ & $0.048(3)$ & $0.003(3)$ & $-0.014(2)$ & -0.006 \\
\hline $\mathrm{C} 10$ & $0.040(3)$ & $0.043(3)$ & $0.063(4)$ & $-0.003(3)$ & $-0.010(3)$ & $0.003(3)$ \\
\hline $\mathrm{C} 11$ & 0.034 & $0.051(3)$ & $0.046(3)$ & -0.004 (3) & $-0.008(2)$ & 0.009 (3) \\
\hline $\mathrm{C} 12$ & $0.033(3)$ & $0.038(3)$ & $0.035(3)$ & $0.005(2)$ & $-0.007(2)$ & $0.004(2)$ \\
\hline $\mathrm{C} 13$ & $0.027(2)$ & $0.044(3)$ & 0.032 & $0.001(2)$ & $0.0016(19)$ & $0.002(2)$ \\
\hline C14 & $0.036(3)$ & $0.050(3)$ & $0.036(3)$ & $-0.007(3)$ & $0.010(2)$ & $0.002(2)$ \\
\hline $\mathrm{C} 15$ & $0.032(3)$ & $0.063(4)$ & $0.051(3)$ & $0.007(3)$ & $0.011(2)$ & $0.005(3)$ \\
\hline $\mathrm{C} 16$ & 0.038 & $0.055(4)$ & $0.040(3)$ & $0.012(3)$ & $0.012(2)$ & -0.002 \\
\hline C17 & $0.032(3)$ & $0.042(3)$ & $0.032(3)$ & $0.000(2)$ & $0.007(2)$ & $-0.001(2)$ \\
\hline C18 & 0.031 & $0.038(3)$ & $0.029(2)$ & $0.001(2)$ & 0.0057 (19) & $0.003(2)$ \\
\hline C19 & $0.038(3)$ & $0.050(3)$ & $0.036(3)$ & $0.002(3)$ & $-0.001(2)$ & $-0.007(2)$ \\
\hline $\mathrm{C} 20$ & $0.059(4)$ & $0.058(4)$ & 0.034 & $-0.005(3)$ & $-0.003(2)$ & 0.011 \\
\hline $\mathrm{C} 21$ & $0.026(2)$ & $0.028(2)$ & $0.025(2)$ & $0.000(2)$ & $0.0004(18)$ & -0.0003 \\
\hline $\mathrm{C} 22$ & $0.026(2)$ & $0.035(3)$ & $0.025(2)$ & $-0.003(2)$ & $0.0080(18)$ & $-0.001(2)$ \\
\hline $\mathrm{C} 23$ & $0.024(2)$ & $0.027(3)$ & 0.039 (3) & $0.0000(19)$ & $0.0082(19)$ & $-0.003(2)$ \\
\hline $\mathrm{C} 24$ & $0.024(2)$ & $0.036(3)$ & $0.036(3)$ & $-0.003(2)$ & $0.0033(19)$ & $0.008(2)$ \\
\hline $\mathrm{C} 25$ & $0.026(2)$ & $0.040(3)$ & $0.027(2)$ & $-0.004(2)$ & $0.0046(19)$ & $0.003(2)$ \\
\hline $\mathrm{C} 26$ & $0.024(2)$ & $0.042(3)$ & $0.026(2)$ & $-0.007(2)$ & $0.0050(18)$ & $-0.005(2)$ \\
\hline $\mathrm{C} 27$ & $0.038(3)$ & $0.050(3)$ & $0.027(3)$ & $0.002(2)$ & $0.012(2)$ & $0.001(2)$ \\
\hline $\mathrm{C} 28$ & $0.067(4)$ & $0.062(4)$ & $0.036(3)$ & $-0.006(3)$ & $0.025(3)$ & $-0.007(3)$ \\
\hline $\mathrm{C} 29$ & $0.093(5)$ & $0.073(4)$ & $0.030(3)$ & $-0.016(4)$ & $0.030(3)$ & $-0.007(3)$ \\
\hline $\mathrm{C} 30$ & $0.095(5)$ & $0.070(4)$ & $0.020(3)$ & $-0.008(4)$ & $0.015(3)$ & -0.008 \\
\hline $\mathrm{C} 31$ & $0.067(4)$ & $0.041(3)$ & $0.028(3)$ & $-0.002(3)$ & $0.008(2)$ & $-0.006(2)$ \\
\hline $\mathrm{C} 32$ & $0.041(3)$ & $0.043(3)$ & $0.024(2)$ & $0.010(2)$ & $0.005(2)$ & $0.002(2)$ \\
\hline $\mathrm{C} 33$ & $0.036(3)$ & $0.035(3)$ & $0.034(3)$ & $0.003(2)$ & $0.009(2)$ & $-0.005(2)$ \\
\hline C34 & $0.047(3)$ & $0.040(3)$ & $0.039(3)$ & $0.011(3)$ & $0.007(2)$ & -0.005 (2) \\
\hline $\mathrm{C} 35$ & $0.067(4)$ & $0.048(4)$ & $0.057(4)$ & $0.029(3)$ & $0.008(3)$ & $-0.014(3)$ \\
\hline C36 & $0.054(4)$ & $0.064(4)$ & $0.046(3)$ & $0.026(3)$ & 0.007 (3) & -0.001 \\
\hline C37 & $0.035(3)$ & $0.048(3)$ & $0.030(3)$ & $0.012(2)$ & $0.007(2)$ & $0.000(2)$ \\
\hline C38 & $0.025(2)$ & $0.040(3)$ & $0.027(2)$ & $0.006(2)$ & $0.0103(18)$ & $0.002(2)$ \\
\hline C39 & $0.028(3)$ & $0.064(4)$ & $0.037(3)$ & $-0.002(3)$ & $0.012(2)$ & $-0.002(3)$ \\
\hline C40 & 0.039 (3) & $0.035(3)$ & 0.031 & $0.002(2)$ & $0.004(2)$ & $0.000(2)$ \\
\hline $\mathrm{C} 41$ & $0.022(2)$ & $0.037(3)$ & $0.033(3)$ & $-0.002(2)$ & $0.0090(18)$ & $-0.008(2)$ \\
\hline $\mathrm{C} 42$ & $0.035(3)$ & $0.035(3)$ & $0.036(3)$ & $0.000(2)$ & $0.016(2)$ & $-0.003(2)$ \\
\hline $\mathrm{C} 43$ & $0.050(3)$ & $0.037(3)$ & $0.059(4)$ & $0.000(3)$ & $0.024(3)$ & $-0.011(3)$ \\
\hline $\mathrm{C} 44$ & $0.073(4)$ & $0.042(4)$ & $0.072(4)$ & $-0.008(3)$ & $0.039(3)$ & -0.023 \\
\hline $\mathrm{C} 45$ & $0.064(4)$ & $0.047(3)$ & $0.036(3)$ & $-0.007(3)$ & $0.025(3)$ & $-0.014(3)$ \\
\hline $\mathrm{C} 46$ & $0.038(3)$ & $0.035(3)$ & $0.035(3)$ & $-0.010(2)$ & $0.013(2)$ & $-0.013(2)$ \\
\hline $\mathrm{C} 47$ & 0.039 & $0.035(3)$ & $0.027(2)$ & $0.001(2)$ & $0.006(2)$ & $0.005(2)$ \\
\hline $\mathrm{C} 48$ & $0.047(3)$ & $0.050(3)$ & $0.035(3)$ & $-0.008(3)$ & $0.019(2)$ & $-0.003(2)$ \\
\hline C49 & $0.058(4)$ & $0.067(4)$ & $0.037(3)$ & $-0.008(3)$ & $0.025(3)$ & -0.001 \\
\hline $\mathrm{C} 50$ & $0.044(3)$ & $0.055(4)$ & $0.042(3)$ & $-0.007(3)$ & $0.015(2)$ & $0.011(3)$ \\
\hline C51 & $0.041(3)$ & $0.045(3)$ & $0.034(3)$ & $-0.003(3)$ & $0.001(2)$ & $-0.001(2)$ \\
\hline C52 & 0.034 & $0.036(3)$ & $0.023(2)$ & $-0.001(2)$ & $0.0043(19)$ & $0.003(2)$ \\
\hline
\end{tabular}




\begin{tabular}{|c|c|c|c|c|c|c|}
\hline $\mathrm{C} 53$ & $0.029(2)$ & $0.043(3)$ & $0.029(2)$ & $0.007(2)$ & $0.0106(19)$ & $0.000(2)$ \\
\hline $\mathrm{C} 54$ & $0.043(3)$ & $0.044(3)$ & $0.033(3)$ & $0.009(2)$ & $0.013(2)$ & $0.010(2)$ \\
\hline C55 & $0.024(3)$ & $0.058(4)$ & $0.054(3)$ & $0.012(3)$ & $0.004(2)$ & $0.012(3)$ \\
\hline C56 & $0.032(3)$ & $0.054(4)$ & 0.050 & $0.001(3)$ & $0.010(2)$ & $0.006(3)$ \\
\hline $\mathrm{C} 57$ & $0.029(3)$ & $0.049(3)$ & $0.031(3)$ & $0.006(2)$ & $0.006(2)$ & $0.006(2)$ \\
\hline $\mathrm{C} 58$ & $0.028(2)$ & $0.037(3)$ & $0.029(2)$ & $0.005(2)$ & $0.0102(19)$ & $-0.003(2)$ \\
\hline C59 & $0.045(3)$ & $0.067(4)$ & $0.027(3)$ & $-0.012(3)$ & $0.006(2)$ & $-0.007(3)$ \\
\hline $\mathrm{C} 60$ & $0.053(3)$ & $0.033(3)$ & $0.035(3)$ & $0.003(2)$ & $0.004(2)$ & $-0.002(2)$ \\
\hline C61 & $0.027(2)$ & $0.034(3)$ & $0.021(2)$ & $-0.002(2)$ & $0.0083(18)$ & $-0.0014(19)$ \\
\hline C62 & $0.029(2)$ & $0.036(3)$ & $0.027(2)$ & $0.000(2)$ & $0.0081(19)$ & $0.002(2)$ \\
\hline C63 & $0.040(3)$ & $0.030(3)$ & $0.026(2)$ & $-0.008(2)$ & $0.013(2)$ & $-0.007(2)$ \\
\hline C64 & $0.033(3)$ & $0.033(3)$ & $0.035(3)$ & $0.005(2)$ & $0.009(2)$ & $0.000(2)$ \\
\hline C65 & $0.033(2)$ & $0.030(3)$ & $0.024(2)$ & $0.002(2)$ & $0.0077(18)$ & $0.005(2)$ \\
\hline C66 & $0.026(2)$ & $0.028(3)$ & $0.022(2)$ & $-0.0020(19)$ & $0.0065(17)$ & $0.0036(19)$ \\
\hline C67 & $0.030(2)$ & $0.042(3)$ & $0.024(2)$ & $0.003(2)$ & $0.0069(18)$ & $-0.004(2)$ \\
\hline C68 & $0.033(3)$ & $0.046(3)$ & $0.028(2)$ & $0.009(2)$ & $0.0038(19)$ & $0.000(2)$ \\
\hline C69 & $0.027(3)$ & $0.066(4)$ & 0.030 & $0.008(3)$ & $0.003(2)$ & $-0.004(3)$ \\
\hline $\mathrm{C} 70$ & $0.027(2)$ & 0.055 & $0.040(3)$ & $-0.001(2)$ & $0.008(2)$ & $-0.010(3)$ \\
\hline C71 & $0.031(3)$ & 0.045 & $0.029(2)$ & $0.001(2)$ & $0.011(2)$ & $0.000(2)$ \\
\hline $\mathrm{C} 72$ & $0.028(2)$ & $0.038(3)$ & $0.024(2)$ & $0.000(2)$ & $0.0025(18)$ & $-0.005(2)$ \\
\hline $\mathrm{C} 73$ & $0.033(3)$ & 0.035 & $0.024(2)$ & $0.007(2)$ & $0.0031(19)$ & $0.002(2)$ \\
\hline $\mathrm{C} 74$ & $0.030(3)$ & $0.034(3)$ & $0.037(3)$ & $-0.003(2)$ & $0.003(2)$ & $0.003(2)$ \\
\hline C75 & $0.040(3)$ & $0.040(3)$ & $0.032(3)$ & $0.006(2)$ & $0.004(2)$ & $-0.007(2)$ \\
\hline C76 & $0.035(3)$ & $0.041(3)$ & $0.027(2)$ & $0.013(2)$ & $0.0050(19)$ & $-0.002(2)$ \\
\hline $\mathrm{C} 77$ & $0.030(2)$ & $0.040(3)$ & $0.024(2)$ & $0.005(2)$ & $0.0035(18)$ & $0.001(2)$ \\
\hline $\mathrm{C} 78$ & $0.029(2)$ & $0.033(3)$ & $0.020(2)$ & $0.006(2)$ & $0.0051(18)$ & $0.0056(19)$ \\
\hline C79 & $0.037(3)$ & $0.042(3)$ & $0.024(2)$ & $0.001(2)$ & 0.007 (2) & $0.003(2)$ \\
\hline $\mathrm{C} 80$ & $0.038(3)$ & $0.056(3)$ & $0.032(3)$ & $0.004(3)$ & 0.007 (2) & $0.009(2)$ \\
\hline $\mathrm{Fe} 1$ & $0.0246(3)$ & $0.0327(4)$ & $0.0226(3)$ & $0.0008(3)$ & $0.0037(3)$ & $-0.0012(3)$ \\
\hline $\mathrm{Fe} 2$ & $0.0281(4)$ & $0.0352(4)$ & $0.0279(4)$ & $-0.0007(3)$ & $0.0058(3)$ & $-0.0004(3)$ \\
\hline N1 & $0.0237(19)$ & $0.030(2)$ & $0.037(2)$ & $-0.0012(17)$ & $0.0041(16)$ & $0.0004(17)$ \\
\hline N2 & $0.026(2)$ & $0.043(3)$ & $0.035(2)$ & $0.0028(19)$ & $-0.0040(17)$ & $0.0025(19)$ \\
\hline N3 & $0.037(2)$ & $0.038(2)$ & $0.034(2)$ & $-0.0011(19)$ & $-0.0020(18)$ & $0.0014(19)$ \\
\hline N4 & $0.0224(18)$ & $0.036(2)$ & $0.0243(19)$ & $0.0062(16)$ & $0.0071(15)$ & $-0.0028(16)$ \\
\hline N5 & $0.031(2)$ & $0.050(3)$ & $0.030(2)$ & -0.0004 (19) & $0.0092(17)$ & $0.0023(19)$ \\
\hline N6 & $0.032(2)$ & $0.037(2)$ & $0.025(2)$ & $0.0025(18)$ & $0.0015(16)$ & $0.0009(17)$ \\
\hline N7 & $0.0236(19)$ & $0.036(2)$ & $0.026(2)$ & $0.0035(17)$ & $0.0058(15)$ & $-0.0040(17)$ \\
\hline N8 & $0.032(2)$ & $0.042(2)$ & $0.025(2)$ & $-0.0081(19)$ & $0.0049(16)$ & $-0.0054(18)$ \\
\hline N9 & $0.036(2)$ & $0.031(2)$ & $0.0237(19)$ & $-0.0001(18)$ & $0.0042(16)$ & $-0.0003(17)$ \\
\hline N10 & $0.0184(17)$ & $0.029(2)$ & $0.028(2)$ & $-0.0028(16)$ & $0.0013(14)$ & $-0.0057(16)$ \\
\hline N11 & $0.031(2)$ & $0.033(2)$ & $0.0239(19)$ & $0.0048(17)$ & $0.0070(15)$ & $0.0022(16)$ \\
\hline N12 & $0.0223(18)$ & $0.034(2)$ & $0.0244(19)$ & $0.0018(16)$ & $0.0031(15)$ & $0.0019(16)$ \\
\hline
\end{tabular}

Geometric parameters $\left(\AA,{ }^{\circ}\right)$

\begin{tabular}{llll}
\hline $\mathrm{C} 1-\mathrm{C} 6$ & $1.401(6)$ & $\mathrm{C} 42-\mathrm{H} 42$ & 0.9500 \\
$\mathrm{C} 1-\mathrm{C} 2$ & $1.406(6)$ & $\mathrm{C} 43-\mathrm{C} 44$ & $1.375(8)$ \\
$\mathrm{C} 1-\mathrm{N} 1$ & $1.426(6)$ & $\mathrm{C} 43-\mathrm{H} 43$ & 0.9500
\end{tabular}




\begin{tabular}{|c|c|c|c|}
\hline $\mathrm{C} 2-\mathrm{C} 3$ & $1.376(6)$ & $\mathrm{C} 44-\mathrm{C} 45$ & $1.390(7)$ \\
\hline $\mathrm{C} 2-\mathrm{H} 2$ & 0.9500 & $\mathrm{C} 44-\mathrm{H} 44$ & 0.9500 \\
\hline $\mathrm{C} 3-\mathrm{C} 4$ & $1.375(7)$ & $\mathrm{C} 45-\mathrm{C} 46$ & $1.386(6)$ \\
\hline $\mathrm{C} 3-\mathrm{H} 3$ & 0.9500 & $\mathrm{C} 45-\mathrm{H} 45$ & 0.9500 \\
\hline $\mathrm{C} 4-\mathrm{C} 5$ & $1.401(6)$ & $\mathrm{C} 46-\mathrm{N} 8$ & $1.432(6)$ \\
\hline $\mathrm{C} 4-\mathrm{H} 4$ & 0.9500 & $\mathrm{C} 47-\mathrm{C} 48$ & $1.384(6)$ \\
\hline $\mathrm{C} 5-\mathrm{C} 6$ & $1.370(7)$ & $\mathrm{C} 47-\mathrm{C} 52$ & $1.394(6)$ \\
\hline $\mathrm{C} 5-\mathrm{H} 5$ & 0.9500 & $\mathrm{C} 47-\mathrm{N} 8$ & $1.446(6)$ \\
\hline $\mathrm{C} 6-\mathrm{N} 2$ & $1.456(5)$ & $\mathrm{C} 48-\mathrm{C} 49$ & $1.378(7)$ \\
\hline $\mathrm{C} 7-\mathrm{C} 8$ & $1.388(7)$ & $\mathrm{C} 48-\mathrm{H} 48$ & 0.9500 \\
\hline $\mathrm{C} 7-\mathrm{C} 12$ & $1.416(7)$ & $\mathrm{C} 49-\mathrm{C} 50$ & $1.373(7)$ \\
\hline $\mathrm{C} 7-\mathrm{N} 2$ & $1.434(6)$ & $\mathrm{C} 49-\mathrm{H} 49$ & 0.9500 \\
\hline $\mathrm{C} 8-\mathrm{C} 9$ & $1.391(7)$ & $\mathrm{C} 50-\mathrm{C} 51$ & $1.381(7)$ \\
\hline $\mathrm{C} 8-\mathrm{H} 8$ & 0.9500 & $\mathrm{C} 50-\mathrm{H} 50$ & 0.9500 \\
\hline $\mathrm{C} 9-\mathrm{C} 10$ & $1.378(8)$ & $\mathrm{C} 51-\mathrm{C} 52$ & $1.401(7)$ \\
\hline C9-H9 & 0.9500 & $\mathrm{C} 51-\mathrm{H} 51$ & 0.9500 \\
\hline $\mathrm{C} 10-\mathrm{C} 11$ & $1.403(7)$ & $\mathrm{C} 52-\mathrm{N} 9$ & $1.476(6)$ \\
\hline $\mathrm{C} 10-\mathrm{H} 10$ & 0.9500 & C53-C54 & $1.386(6)$ \\
\hline $\mathrm{C} 11-\mathrm{C} 12$ & $1.410(7)$ & $\mathrm{C} 53-\mathrm{C} 58$ & $1.401(6)$ \\
\hline C11-H11 & 0.9500 & $\mathrm{C} 53-\mathrm{N} 9$ & $1.458(6)$ \\
\hline $\mathrm{C} 12-\mathrm{N} 3$ & $1.395(6)$ & $\mathrm{C} 54-\mathrm{C} 55$ & $1.382(7)$ \\
\hline $\mathrm{C} 13-\mathrm{C} 14$ & $1.377(7)$ & C54-H54 & 0.9500 \\
\hline $\mathrm{C} 13-\mathrm{C} 18$ & $1.411(7)$ & $\mathrm{C} 55-\mathrm{C} 56$ & $1.382(7)$ \\
\hline $\mathrm{C} 13-\mathrm{N} 3$ & $1.456(6)$ & $\mathrm{C} 55-\mathrm{H} 55$ & 0.9500 \\
\hline $\mathrm{C} 14-\mathrm{C} 15$ & $1.381(7)$ & $\mathrm{C} 56-\mathrm{C} 57$ & $1.383(6)$ \\
\hline C14-H14 & 0.9500 & C56-H56 & 0.9500 \\
\hline $\mathrm{C} 15-\mathrm{C} 16$ & $1.397(7)$ & $\mathrm{C} 57-\mathrm{C} 58$ & $1.385(6)$ \\
\hline C15-H15 & 0.9500 & C57-H57 & 0.9500 \\
\hline $\mathrm{C} 16-\mathrm{C} 17$ & $1.372(7)$ & $\mathrm{C} 58-\mathrm{N} 7$ & $1.446(5)$ \\
\hline $\mathrm{C} 16-\mathrm{H} 16$ & 0.9500 & $\mathrm{C} 59-\mathrm{N} 8$ & $1.461(5)$ \\
\hline $\mathrm{C} 17-\mathrm{C} 18$ & $1.403(6)$ & С59-H59A & 0.9800 \\
\hline C17-H17 & 0.9500 & С $59-\mathrm{H} 59 \mathrm{~B}$ & 0.9800 \\
\hline $\mathrm{C} 18-\mathrm{N} 1$ & $1.386(6)$ & С59-H59C & 0.9800 \\
\hline $\mathrm{C} 19-\mathrm{N} 2$ & $1.468(6)$ & $\mathrm{C} 60-\mathrm{N} 9$ & $1.492(6)$ \\
\hline C19-H19A & 0.9800 & C60-H60A & 0.9800 \\
\hline C19-H19B & 0.9800 & С60-H60B & 0.9800 \\
\hline C19-H19C & 0.9800 & C60-H60C & 0.9800 \\
\hline $\mathrm{C} 20-\mathrm{N} 3$ & $1.459(6)$ & $\mathrm{C} 61-\mathrm{N} 10$ & $1.395(5)$ \\
\hline $\mathrm{C} 20-\mathrm{H} 20 \mathrm{~A}$ & 0.9800 & $\mathrm{C} 61-\mathrm{C} 62$ & $1.405(6)$ \\
\hline $\mathrm{C} 20-\mathrm{H} 20 \mathrm{~B}$ & 0.9800 & C61-C66 & $1.414(6)$ \\
\hline $\mathrm{C} 20-\mathrm{H} 20 \mathrm{C}$ & 0.9800 & $\mathrm{C} 62-\mathrm{C} 63$ & $1.372(6)$ \\
\hline $\mathrm{C} 21-\mathrm{C} 22$ & $1.404(6)$ & C62-H62 & 0.9500 \\
\hline $\mathrm{C} 21-\mathrm{C} 26$ & $1.417(6)$ & $\mathrm{C} 63-\mathrm{C} 64$ & $1.389(6)$ \\
\hline $\mathrm{C} 21-\mathrm{N} 4$ & $1.436(5)$ & C63-H63 & 0.9500 \\
\hline $\mathrm{C} 21-\mathrm{Fe} 2$ & $2.426(4)$ & $\mathrm{C} 64-\mathrm{C} 65$ & $1.389(6)$ \\
\hline $\mathrm{C} 22-\mathrm{C} 23$ & $1.388(6)$ & C64-H64 & 0.9500 \\
\hline $\mathrm{C} 22-\mathrm{H} 22$ & 0.9500 & $\mathrm{C} 65-\mathrm{C} 66$ & $1.375(6)$ \\
\hline $\mathrm{C} 23-\mathrm{C} 24$ & $1.384(6)$ & $\mathrm{C} 65-\mathrm{H} 65$ & 0.9500 \\
\hline
\end{tabular}




\begin{tabular}{|c|c|c|c|}
\hline $\mathrm{C} 23-\mathrm{H} 23$ & 0.9500 & C66-N11 & $1.449(5)$ \\
\hline $\mathrm{C} 24-\mathrm{C} 25$ & $1.386(6)$ & C67-C68 & $1.403(6)$ \\
\hline $\mathrm{C} 24-\mathrm{H} 24$ & 0.9500 & $\mathrm{C} 67-\mathrm{C} 72$ & $1.407(6)$ \\
\hline $\mathrm{C} 25-\mathrm{C} 26$ & $1.376(6)$ & C67-N11 & $1.421(6)$ \\
\hline $\mathrm{C} 25-\mathrm{H} 25$ & 0.9500 & C68-C69 & $1.375(7)$ \\
\hline $\mathrm{C} 26-\mathrm{N} 5$ & $1.445(6)$ & C68-H68 & 0.9500 \\
\hline $\mathrm{C} 27-\mathrm{C} 28$ & $1.394(7)$ & $\mathrm{C} 69-\mathrm{C} 70$ & $1.381(7)$ \\
\hline $\mathrm{C} 27-\mathrm{N} 5$ & $1.398(6)$ & C69-H69 & 0.9500 \\
\hline $\mathrm{C} 27-\mathrm{C} 32$ & $1.419(7)$ & $\mathrm{C} 70-\mathrm{C} 71$ & $1.391(6)$ \\
\hline $\mathrm{C} 28-\mathrm{C} 29$ & $1.381(7)$ & $\mathrm{C} 70-\mathrm{H} 70$ & 0.9500 \\
\hline $\mathrm{C} 28-\mathrm{H} 28$ & 0.9500 & C71-C72 & $1.387(6)$ \\
\hline $\mathrm{C} 29-\mathrm{C} 30$ & $1.373(8)$ & C71-H71 & 0.9500 \\
\hline С29- & 0.9500 & $\mathrm{C} 72-\mathrm{N} 12$ & $1.422(5)$ \\
\hline $\mathrm{C} 30-\mathrm{C} 31$ & $1.393(7)$ & C73-C74 & $1.392(6)$ \\
\hline C $30-\mathrm{H} 30$ & 0.9500 & $\mathrm{C} 73-\mathrm{C} 78$ & $1.414(6)$ \\
\hline $\mathrm{C} 31-\mathrm{C} 32$ & $1.390(6)$ & $\mathrm{C} 73-\mathrm{N} 12$ & $1.428(6)$ \\
\hline C $31-\mathrm{H} 31$ & 0.9500 & $\mathrm{C} 74-\mathrm{C} 75$ & $1.383(7)$ \\
\hline $\mathrm{C} 32-\mathrm{N} 6$ & $1.427(6)$ & $\mathrm{C} 74-\mathrm{H} 74$ & 0.9500 \\
\hline C $33-\mathrm{C} 34$ & $1.392(6)$ & $\mathrm{C} 75-\mathrm{C} 76$ & $1.381(7)$ \\
\hline $\mathrm{C} 33-\mathrm{C} 38$ & $1.401(6)$ & C75-H75 & 0.9500 \\
\hline $\mathrm{C} 33-\mathrm{N} 6$ & $1.444(6)$ & C76-C77 & $1.382(6)$ \\
\hline C $34-C 35$ & $1.390(7)$ & C76-H76 & 0.9500 \\
\hline C34-H34 & 0.9500 & $\mathrm{C} 77-\mathrm{C} 78$ & $1.423(6)$ \\
\hline $\mathrm{C} 35-\mathrm{C} 36$ & $1.392(7)$ & C77-H77 & 0.9500 \\
\hline C $35-\mathrm{H} 35$ & 0.9500 & $\mathrm{C} 78-\mathrm{N} 10$ & $1.393(5)$ \\
\hline C36-C37 & $1.373(7)$ & C79-N11 & $1.456(5)$ \\
\hline C36-H36 & 0.9500 & C79-H79A & 0.9800 \\
\hline C $37-\mathrm{C} 38$ & $1.413(6)$ & С $79-\mathrm{H} 79 \mathrm{~B}$ & 0.9800 \\
\hline C37- H37 & 0.9500 & C79-H79C & 0.9800 \\
\hline $\mathrm{C} 38-\mathrm{N} 4$ & $1.426(6)$ & $\mathrm{C} 80-\mathrm{N} 12$ & $1.454(6)$ \\
\hline C39-N5 & $1.470(6)$ & $\mathrm{C} 80-\mathrm{H} 80 \mathrm{~A}$ & 0.9800 \\
\hline С39- H39A & 0.9800 & $\mathrm{C} 80-\mathrm{H} 80 \mathrm{~B}$ & 0.9800 \\
\hline С $39-\mathrm{H} 39 \mathrm{~B}$ & 0.9800 & $\mathrm{C} 80-\mathrm{H} 80 \mathrm{C}$ & 0.9800 \\
\hline С $39-\mathrm{H} 39 \mathrm{C}$ & 0.9800 & $\mathrm{Fe} 1-\mathrm{N} 10$ & $1.992(3)$ \\
\hline $\mathrm{C} 40-\mathrm{N} 6$ & $1.458(6)$ & $\mathrm{Fe} 1-\mathrm{N} 7$ & $2.091(4)$ \\
\hline $\mathrm{C} 40-\mathrm{H} 40 \mathrm{~A}$ & 0.9800 & $\mathrm{Fe} 1-\mathrm{N} 4$ & $2.154(4)$ \\
\hline $\mathrm{C} 40-\mathrm{H} 40 \mathrm{~B}$ & 0.9800 & $\mathrm{Fe} 1-\mathrm{N} 9$ & $2.245(3)$ \\
\hline $\mathrm{C} 40-\mathrm{H} 40 \mathrm{C}$ & 0.9800 & $\mathrm{Fe} 1-\mathrm{Fe} 2$ & $2.9121(10)$ \\
\hline $\mathrm{C} 41-\mathrm{C} 42$ & $1.396(6)$ & $\mathrm{Fe} 2-\mathrm{N} 1$ & $1.947(4)$ \\
\hline $\mathrm{C} 41-\mathrm{C} 46$ & $1.407(6)$ & $\mathrm{Fe} 2-\mathrm{N} 7$ & $2.061(4)$ \\
\hline $\mathrm{C} 41-\mathrm{N} 7$ & $1.429(5)$ & $\mathrm{Fe} 2-\mathrm{N} 4$ & $2.068(4)$ \\
\hline $\mathrm{C} 42-\mathrm{C} 43$ & $1.367(6)$ & & \\
\hline $\mathrm{C} 6-\mathrm{C} 1-\mathrm{C} 2$ & $117.3(4)$ & $\mathrm{C} 50-\mathrm{C} 51-\mathrm{H} 51$ & 119.4 \\
\hline $\mathrm{C} 6-\mathrm{C} 1-\mathrm{N} 1$ & $124.8(4)$ & C52-C51-H51 & 119.4 \\
\hline $\mathrm{C} 2-\mathrm{C} 1-\mathrm{N} 1$ & $117.5(4)$ & $\mathrm{C} 47-\mathrm{C} 52-\mathrm{C} 51$ & $118.0(5)$ \\
\hline $\mathrm{C} 3-\mathrm{C} 2-\mathrm{C} 1$ & $121.2(4)$ & $\mathrm{C} 47-\mathrm{C} 52-\mathrm{N} 9$ & $124.9(4)$ \\
\hline $\mathrm{C} 3-\mathrm{C} 2-\mathrm{H} 2$ & 119.4 & $\mathrm{C} 51-\mathrm{C} 52-\mathrm{N} 9$ & $117.0(4)$ \\
\hline
\end{tabular}




\begin{tabular}{|c|c|c|c|}
\hline $\mathrm{C} 1-\mathrm{C} 2-\mathrm{H} 2$ & 119.4 & $\mathrm{C} 54-\mathrm{C} 53-\mathrm{C} 58$ & $120.2(5)$ \\
\hline $\mathrm{C} 4-\mathrm{C} 3-\mathrm{C} 2$ & $121.1(4)$ & $\mathrm{C} 54-\mathrm{C} 53-\mathrm{N} 9$ & $122.0(4)$ \\
\hline $\mathrm{C} 4-\mathrm{C} 3-\mathrm{H} 3$ & 119.4 & $\mathrm{C} 58-\mathrm{C} 53-\mathrm{N} 9$ & $117.7(4)$ \\
\hline $\mathrm{C} 2-\mathrm{C} 3-\mathrm{H} 3$ & 119.4 & $\mathrm{C} 55-\mathrm{C} 54-\mathrm{C} 53$ & $119.8(5)$ \\
\hline $\mathrm{C} 3-\mathrm{C} 4-\mathrm{C} 5$ & $118.1(5)$ & $\mathrm{C} 55-\mathrm{C} 54-\mathrm{H} 54$ & 120.1 \\
\hline $\mathrm{C} 3-\mathrm{C} 4-\mathrm{H} 4$ & 120.9 & $\mathrm{C} 53-\mathrm{C} 54-\mathrm{H} 54$ & 120.1 \\
\hline $\mathrm{C} 5-\mathrm{C} 4-\mathrm{H} 4$ & 120.9 & $\mathrm{C} 56-\mathrm{C} 55-\mathrm{C} 54$ & $120.5(5)$ \\
\hline $\mathrm{C} 6-\mathrm{C} 5-\mathrm{C} 4$ & $121.4(5)$ & $\mathrm{C} 56-\mathrm{C} 55-\mathrm{H} 55$ & 119.8 \\
\hline $\mathrm{C} 6-\mathrm{C} 5-\mathrm{H} 5$ & 119.3 & $\mathrm{C} 54-\mathrm{C} 55-\mathrm{H} 55$ & 119.8 \\
\hline $\mathrm{C} 4-\mathrm{C} 5-\mathrm{H} 5$ & 119.3 & $\mathrm{C} 55-\mathrm{C} 56-\mathrm{C} 57$ & $119.7(5)$ \\
\hline $\mathrm{C} 5-\mathrm{C} 6-\mathrm{C} 1$ & $120.8(4)$ & $\mathrm{C} 55-\mathrm{C} 56-\mathrm{H} 56$ & 120.2 \\
\hline $\mathrm{C} 5-\mathrm{C} 6-\mathrm{N} 2$ & $118.6(4)$ & $\mathrm{C} 57-\mathrm{C} 56-\mathrm{H} 56$ & 120.2 \\
\hline $\mathrm{C} 1-\mathrm{C} 6-\mathrm{N} 2$ & $120.7(4)$ & $\mathrm{C} 56-\mathrm{C} 57-\mathrm{C} 58$ & $120.9(5)$ \\
\hline $\mathrm{C} 8-\mathrm{C} 7-\mathrm{C} 12$ & $120.0(5)$ & $\mathrm{C} 56-\mathrm{C} 57-\mathrm{H} 57$ & 119.5 \\
\hline $\mathrm{C} 8-\mathrm{C} 7-\mathrm{N} 2$ & $124.0(5)$ & $\mathrm{C} 58-\mathrm{C} 57-\mathrm{H} 57$ & 119.5 \\
\hline $\mathrm{C} 12-\mathrm{C} 7-\mathrm{N} 2$ & $115.9(4)$ & $\mathrm{C} 57-\mathrm{C} 58-\mathrm{C} 53$ & $118.9(4)$ \\
\hline $\mathrm{C} 7-\mathrm{C} 8-\mathrm{C} 9$ & $121.2(5)$ & $\mathrm{C} 57-\mathrm{C} 58-\mathrm{N} 7$ & $122.0(4)$ \\
\hline $\mathrm{C} 7-\mathrm{C} 8-\mathrm{H} 8$ & 119.4 & $\mathrm{C} 53-\mathrm{C} 58-\mathrm{N} 7$ & $119.2(4)$ \\
\hline $\mathrm{C} 9-\mathrm{C} 8-\mathrm{H} 8$ & 119.4 & $\mathrm{~N} 8-\mathrm{C} 59-\mathrm{H} 59 \mathrm{~A}$ & 109.5 \\
\hline $\mathrm{C} 10-\mathrm{C} 9-\mathrm{C} 8$ & $119.4(5)$ & $\mathrm{N} 8-\mathrm{C} 59-\mathrm{H} 59 \mathrm{~B}$ & 109.5 \\
\hline $\mathrm{C} 10-\mathrm{C} 9-\mathrm{H} 9$ & 120.3 & $\mathrm{H} 59 \mathrm{~A}-\mathrm{C} 59-\mathrm{H} 59 \mathrm{~B}$ & 109.5 \\
\hline $\mathrm{C} 8-\mathrm{C} 9-\mathrm{H} 9$ & 120.3 & $\mathrm{~N} 8-\mathrm{C} 59-\mathrm{H} 59 \mathrm{C}$ & 109.5 \\
\hline $\mathrm{C} 9-\mathrm{C} 10-\mathrm{C} 11$ & $120.5(5)$ & $\mathrm{H} 59 \mathrm{~A}-\mathrm{C} 59-\mathrm{H} 59 \mathrm{C}$ & 109.5 \\
\hline $\mathrm{C} 9-\mathrm{C} 10-\mathrm{H} 10$ & 119.8 & $\mathrm{H} 59 \mathrm{~B}-\mathrm{C} 59-\mathrm{H} 59 \mathrm{C}$ & 109.5 \\
\hline $\mathrm{C} 11-\mathrm{C} 10-\mathrm{H} 10$ & 119.8 & $\mathrm{~N} 9-\mathrm{C} 60-\mathrm{H} 60 \mathrm{~A}$ & 109.5 \\
\hline $\mathrm{C} 10-\mathrm{C} 11-\mathrm{C} 12$ & $120.5(5)$ & N9- $\mathrm{C} 60-\mathrm{H} 60 \mathrm{~B}$ & 109.5 \\
\hline $\mathrm{C} 10-\mathrm{C} 11-\mathrm{H} 11$ & 119.7 & $\mathrm{H} 60 \mathrm{~A}-\mathrm{C} 60-\mathrm{H} 60 \mathrm{~B}$ & 109.5 \\
\hline $\mathrm{C} 12-\mathrm{C} 11-\mathrm{H} 11$ & 119.7 & N9- $\mathrm{C} 60-\mathrm{H} 60 \mathrm{C}$ & 109.5 \\
\hline $\mathrm{N} 3-\mathrm{C} 12-\mathrm{C} 11$ & $123.3(5)$ & $\mathrm{H} 60 \mathrm{~A}-\mathrm{C} 60-\mathrm{H} 60 \mathrm{C}$ & 109.5 \\
\hline $\mathrm{N} 3-\mathrm{C} 12-\mathrm{C} 7$ & $118.7(4)$ & $\mathrm{H} 60 \mathrm{~B}-\mathrm{C} 60-\mathrm{H} 60 \mathrm{C}$ & 109.5 \\
\hline $\mathrm{C} 11-\mathrm{C} 12-\mathrm{C} 7$ & $118.0(5)$ & $\mathrm{N} 10-\mathrm{C} 61-\mathrm{C} 62$ & $118.5(4)$ \\
\hline $\mathrm{C} 14-\mathrm{C} 13-\mathrm{C} 18$ & $120.8(5)$ & $\mathrm{N} 10-\mathrm{C} 61-\mathrm{C} 66$ & $124.5(4)$ \\
\hline $\mathrm{C} 14-\mathrm{C} 13-\mathrm{N} 3$ & $118.4(4)$ & C62-C61-C66 & $116.5(4)$ \\
\hline $\mathrm{C} 18-\mathrm{C} 13-\mathrm{N} 3$ & $120.7(4)$ & $\mathrm{C} 63-\mathrm{C} 62-\mathrm{C} 61$ & $122.9(4)$ \\
\hline $\mathrm{C} 13-\mathrm{C} 14-\mathrm{C} 15$ & $121.9(5)$ & $\mathrm{C} 63-\mathrm{C} 62-\mathrm{H} 62$ & 118.5 \\
\hline $\mathrm{C} 13-\mathrm{C} 14-\mathrm{H} 14$ & 119.1 & $\mathrm{C} 61-\mathrm{C} 62-\mathrm{H} 62$ & 118.5 \\
\hline $\mathrm{C} 15-\mathrm{C} 14-\mathrm{H} 14$ & 119.1 & $\mathrm{C} 62-\mathrm{C} 63-\mathrm{C} 64$ & $120.1(4)$ \\
\hline $\mathrm{C} 14-\mathrm{C} 15-\mathrm{C} 16$ & $118.2(5)$ & $\mathrm{C} 62-\mathrm{C} 63-\mathrm{H} 63$ & 120.0 \\
\hline C14-C15-H15 & 120.9 & C64-C63-H63 & 120.0 \\
\hline $\mathrm{C} 16-\mathrm{C} 15-\mathrm{H} 15$ & 120.9 & $\mathrm{C} 65-\mathrm{C} 64-\mathrm{C} 63$ & $117.8(4)$ \\
\hline $\mathrm{C} 17-\mathrm{C} 16-\mathrm{C} 15$ & $120.1(5)$ & $\mathrm{C} 65-\mathrm{C} 64-\mathrm{H} 64$ & 121.1 \\
\hline $\mathrm{C} 17-\mathrm{C} 16-\mathrm{H} 16$ & 119.9 & $\mathrm{C} 63-\mathrm{C} 64-\mathrm{H} 64$ & 121.1 \\
\hline $\mathrm{C} 15-\mathrm{C} 16-\mathrm{H} 16$ & 119.9 & $\mathrm{C} 66-\mathrm{C} 65-\mathrm{C} 64$ & $122.9(4)$ \\
\hline $\mathrm{C} 16-\mathrm{C} 17-\mathrm{C} 18$ & $122.6(5)$ & $\mathrm{C} 66-\mathrm{C} 65-\mathrm{H} 65$ & 118.6 \\
\hline $\mathrm{C} 16-\mathrm{C} 17-\mathrm{H} 17$ & 118.7 & $\mathrm{C} 64-\mathrm{C} 65-\mathrm{H} 65$ & 118.6 \\
\hline $\mathrm{C} 18-\mathrm{C} 17-\mathrm{H} 17$ & 118.7 & $\mathrm{C} 65-\mathrm{C} 66-\mathrm{C} 61$ & $119.8(4)$ \\
\hline $\mathrm{N} 1-\mathrm{C} 18-\mathrm{C} 17$ & $117.5(4)$ & $\mathrm{C} 65-\mathrm{C} 66-\mathrm{N} 11$ & $119.2(4)$ \\
\hline $\mathrm{N} 1-\mathrm{C} 18-\mathrm{C} 13$ & $126.2(4)$ & $\mathrm{C} 61-\mathrm{C} 66-\mathrm{N} 11$ & $121.0(4)$ \\
\hline
\end{tabular}




\begin{tabular}{|c|c|}
\hline $\mathrm{C} 17-\mathrm{C} 18-\mathrm{C} 13$ & $116.2(4)$ \\
\hline $\mathrm{N} 2-\mathrm{C} 19-\mathrm{H} 19 \mathrm{~A}$ & 109.5 \\
\hline $\mathrm{N} 2-\mathrm{C} 19-\mathrm{H} 19 \mathrm{~B}$ & 109.5 \\
\hline $\mathrm{H} 19 \mathrm{~A}-\mathrm{C} 19-\mathrm{H} 19 \mathrm{~B}$ & 109.5 \\
\hline $\mathrm{N} 2-\mathrm{C} 19-\mathrm{H} 19 \mathrm{C}$ & 109.5 \\
\hline $\mathrm{H} 19 \mathrm{~A}-\mathrm{C} 19-\mathrm{H} 19 \mathrm{C}$ & 109.5 \\
\hline $\mathrm{H} 19 \mathrm{~B}-\mathrm{C} 19-\mathrm{H} 19 \mathrm{C}$ & 109.5 \\
\hline $\mathrm{N} 3-\mathrm{C} 20-\mathrm{H} 20 \mathrm{~A}$ & 109.5 \\
\hline $\mathrm{N} 3-\mathrm{C} 20-\mathrm{H} 20 \mathrm{~B}$ & 109.5 \\
\hline $\mathrm{H} 20 \mathrm{~A}-\mathrm{C} 20-\mathrm{H} 20 \mathrm{~B}$ & 109.5 \\
\hline $\mathrm{N} 3-\mathrm{C} 20-\mathrm{H} 20 \mathrm{C}$ & 109.5 \\
\hline $\mathrm{H} 20 \mathrm{~A}-\mathrm{C} 20-\mathrm{H} 20 \mathrm{C}$ & 109.5 \\
\hline $\mathrm{H} 20 \mathrm{~B}-\mathrm{C} 20-\mathrm{H} 20 \mathrm{C}$ & 109.5 \\
\hline $\mathrm{C} 22-\mathrm{C} 21-\mathrm{C} 26$ & $117.8(4)$ \\
\hline $\mathrm{C} 22-\mathrm{C} 21-\mathrm{N} 4$ & $117.8(4)$ \\
\hline $\mathrm{C} 26-\mathrm{C} 21-\mathrm{N} 4$ & $124.3(4)$ \\
\hline $\mathrm{C} 22-\mathrm{C} 21-\mathrm{Fe} 2$ & $95.9(3)$ \\
\hline $\mathrm{C} 26-\mathrm{C} 21-\mathrm{Fe} 2$ & $112.8(3)$ \\
\hline $\mathrm{N} 4-\mathrm{C} 21-\mathrm{Fe} 2$ & $58.2(2)$ \\
\hline $\mathrm{C} 23-\mathrm{C} 22-\mathrm{C} 21$ & $120.9(4)$ \\
\hline $\mathrm{C} 23-\mathrm{C} 22-\mathrm{H} 22$ & 119.5 \\
\hline $\mathrm{C} 21-\mathrm{C} 22-\mathrm{H} 22$ & 119.5 \\
\hline $\mathrm{C} 24-\mathrm{C} 23-\mathrm{C} 22$ & $120.6(4)$ \\
\hline $\mathrm{C} 24-\mathrm{C} 23-\mathrm{H} 23$ & 119.7 \\
\hline $\mathrm{C} 22-\mathrm{C} 23-\mathrm{H} 23$ & 119.7 \\
\hline $\mathrm{C} 23-\mathrm{C} 24-\mathrm{C} 25$ & $118.9(4)$ \\
\hline $\mathrm{C} 23-\mathrm{C} 24-\mathrm{H} 24$ & 120.6 \\
\hline $\mathrm{C} 25-\mathrm{C} 24-\mathrm{H} 24$ & 120.6 \\
\hline $\mathrm{C} 26-\mathrm{C} 25-\mathrm{C} 24$ & $121.8(4)$ \\
\hline $\mathrm{C} 26-\mathrm{C} 25-\mathrm{H} 25$ & 119.1 \\
\hline $\mathrm{C} 24-\mathrm{C} 25-\mathrm{H} 25$ & 119.1 \\
\hline $\mathrm{C} 25-\mathrm{C} 26-\mathrm{C} 21$ & $119.9(4)$ \\
\hline $\mathrm{C} 25-\mathrm{C} 26-\mathrm{N} 5$ & 120.3 \\
\hline $\mathrm{C} 21-\mathrm{C} 26-\mathrm{N} 5$ & $119.7(4)$ \\
\hline $\mathrm{C} 28-\mathrm{C} 27-\mathrm{N} 5$ & $124.0(5)$ \\
\hline $\mathrm{C} 28-\mathrm{C} 27-\mathrm{C} 32$ & $118.9(4)$ \\
\hline $\mathrm{N} 5-\mathrm{C} 27-\mathrm{C} 32$ & $117.1(4)$ \\
\hline $\mathrm{C} 29-\mathrm{C} 28-\mathrm{C} 27$ & $120.5(6)$ \\
\hline $\mathrm{C} 29-\mathrm{C} 28-\mathrm{H} 28$ & 119.8 \\
\hline $\mathrm{C} 27-\mathrm{C} 28-\mathrm{H} 28$ & 119.8 \\
\hline $\mathrm{C} 30-\mathrm{C} 29-\mathrm{C} 28$ & $121.2(5)$ \\
\hline $\mathrm{C} 30-\mathrm{C} 29-\mathrm{H} 29$ & 119.4 \\
\hline $\mathrm{C} 28-\mathrm{C} 29-\mathrm{H} 29$ & 119.4 \\
\hline $\mathrm{C} 29-\mathrm{C} 30-\mathrm{C} 31$ & $119.1(5)$ \\
\hline $\mathrm{C} 29-\mathrm{C} 30-\mathrm{H} 30$ & 120.5 \\
\hline $\mathrm{C} 31-\mathrm{C} 30-\mathrm{H} 30$ & 120.5 \\
\hline $\mathrm{C} 32-\mathrm{C} 31-\mathrm{C} 30$ & $121.2(5)$ \\
\hline $\mathrm{C} 32-\mathrm{C} 31-\mathrm{H} 31$ & 119.4 \\
\hline
\end{tabular}

\begin{tabular}{|c|c|}
\hline $\mathrm{C} 68-\mathrm{C} 67-\mathrm{C} 72$ & $119.0(4)$ \\
\hline $\mathrm{C} 68-\mathrm{C} 67-\mathrm{N} 11$ & $123.1(4)$ \\
\hline $\mathrm{C} 72-\mathrm{C} 67-\mathrm{N} 11$ & $117.9(4)$ \\
\hline C69-C68-C67 & $120.4(5)$ \\
\hline $\mathrm{C} 69-\mathrm{C} 68-\mathrm{H} 68$ & 119.8 \\
\hline $\mathrm{C} 67-\mathrm{C} 68-\mathrm{H} 68$ & 119.8 \\
\hline $\mathrm{C} 68-\mathrm{C} 69-\mathrm{C} 70$ & $120.9(4)$ \\
\hline $\mathrm{C} 68-\mathrm{C} 69-\mathrm{H} 69$ & 119.5 \\
\hline $\mathrm{C} 70-\mathrm{C} 69-\mathrm{H} 69$ & 119.5 \\
\hline $\mathrm{C} 69-\mathrm{C} 70-\mathrm{C} 71$ & $119.2(5)$ \\
\hline $\mathrm{C} 69-\mathrm{C} 70-\mathrm{H} 70$ & 120.4 \\
\hline $\mathrm{C} 71-\mathrm{C} 70-\mathrm{H} 70$ & 120.4 \\
\hline $\mathrm{C} 72-\mathrm{C} 71-\mathrm{C} 70$ & $121.2(5)$ \\
\hline $\mathrm{C} 72-\mathrm{C} 71-\mathrm{H} 71$ & 119.4 \\
\hline $\mathrm{C} 70-\mathrm{C} 71-\mathrm{H} 71$ & 119.4 \\
\hline $\mathrm{C} 71-\mathrm{C} 72-\mathrm{C} 67$ & $119.2(4)$ \\
\hline $\mathrm{C} 71-\mathrm{C} 72-\mathrm{N} 12$ & $122.8(4)$ \\
\hline $\mathrm{C} 67-\mathrm{C} 72-\mathrm{N} 12$ & $118.0(4)$ \\
\hline $\mathrm{C} 74-\mathrm{C} 73-\mathrm{C} 78$ & $119.3(4)$ \\
\hline $\mathrm{C} 74-\mathrm{C} 73-\mathrm{N} 12$ & $119.1(4)$ \\
\hline $\mathrm{C} 78-\mathrm{C} 73-\mathrm{N} 12$ & $121.6(4)$ \\
\hline $\mathrm{C} 75-\mathrm{C} 74-\mathrm{C} 73$ & $123.9(5)$ \\
\hline $\mathrm{C} 75-\mathrm{C} 74-\mathrm{H} 74$ & 118.0 \\
\hline $\mathrm{C} 73-\mathrm{C} 74-\mathrm{H} 74$ & 118.0 \\
\hline $\mathrm{C} 76-\mathrm{C} 75-\mathrm{C} 74$ & $117.0(5)$ \\
\hline $\mathrm{C} 76-\mathrm{C} 75-\mathrm{H} 75$ & 121.5 \\
\hline $\mathrm{C} 74-\mathrm{C} 75-\mathrm{H} 75$ & 121.5 \\
\hline $\mathrm{C} 75-\mathrm{C} 76-\mathrm{C} 77$ & $121.2(5)$ \\
\hline $\mathrm{C} 75-\mathrm{C} 76-\mathrm{H} 76$ & 119.4 \\
\hline $\mathrm{C} 77-\mathrm{C} 76-\mathrm{H} 76$ & 119.4 \\
\hline $\mathrm{C} 76-\mathrm{C} 77-\mathrm{C} 78$ & $122.3(4)$ \\
\hline $\mathrm{C} 76-\mathrm{C} 77-\mathrm{H} 77$ & 118.9 \\
\hline $\mathrm{C} 78-\mathrm{C} 77-\mathrm{H} 77$ & 118.9 \\
\hline $\mathrm{N} 10-\mathrm{C} 78-\mathrm{C} 73$ & $125.8(4)$ \\
\hline $\mathrm{N} 10-\mathrm{C} 78-\mathrm{C} 77$ & $117.9(4)$ \\
\hline $\mathrm{C} 73-\mathrm{C} 78-\mathrm{C} 77$ & $116.3(4)$ \\
\hline $\mathrm{N} 11-\mathrm{C} 79-\mathrm{H} 79 \mathrm{~A}$ & 109.5 \\
\hline $\mathrm{N} 11-\mathrm{C} 79-\mathrm{H} 79 \mathrm{~B}$ & 109.5 \\
\hline $\mathrm{H} 79 \mathrm{~A}-\mathrm{C} 79-\mathrm{H} 79 \mathrm{~B}$ & 109.5 \\
\hline $\mathrm{N} 11-\mathrm{C} 79-\mathrm{H} 79 \mathrm{C}$ & 109.5 \\
\hline $\mathrm{H} 79 \mathrm{~A}-\mathrm{C} 79-\mathrm{H} 79 \mathrm{C}$ & 109.5 \\
\hline $\mathrm{H} 79 \mathrm{~B}-\mathrm{C} 79-\mathrm{H} 79 \mathrm{C}$ & 109.5 \\
\hline $\mathrm{N} 12-\mathrm{C} 80-\mathrm{H} 80 \mathrm{~A}$ & 109.5 \\
\hline $\mathrm{N} 12-\mathrm{C} 80-\mathrm{H} 80 \mathrm{~B}$ & 109.5 \\
\hline $\mathrm{H} 80 \mathrm{~A}-\mathrm{C} 80-\mathrm{H} 80 \mathrm{~B}$ & 109.5 \\
\hline $\mathrm{N} 12-\mathrm{C} 80-\mathrm{H} 80 \mathrm{C}$ & 109.5 \\
\hline $\mathrm{H} 80 \mathrm{~A}-\mathrm{C} 80-\mathrm{H} 80 \mathrm{C}$ & 109.5 \\
\hline $\mathrm{H} 80 \mathrm{~B}-\mathrm{C} 80-\mathrm{H} 80 \mathrm{C}$ & 109.5 \\
\hline
\end{tabular}




\begin{tabular}{|c|c|c|c|}
\hline $\mathrm{C} 30-\mathrm{C} 31-\mathrm{H} 31$ & 119.4 & $\mathrm{~N} 10-\mathrm{Fe} 1-\mathrm{N} 7$ & $139.63(14)$ \\
\hline $\mathrm{C} 31-\mathrm{C} 32-\mathrm{C} 27$ & $119.0(5)$ & $\mathrm{N} 10-\mathrm{Fe} 1-\mathrm{N} 4$ & $115.65(13)$ \\
\hline $\mathrm{C} 31-\mathrm{C} 32-\mathrm{N} 6$ & $124.6(5)$ & $\mathrm{N} 7-\mathrm{Fe} 1-\mathrm{N} 4$ & $89.81(14)$ \\
\hline $\mathrm{C} 27-\mathrm{C} 32-\mathrm{N} 6$ & $116.4(4)$ & $\mathrm{N} 10-\mathrm{Fe} 1-\mathrm{N} 9$ & $107.53(14)$ \\
\hline $\mathrm{C} 34-\mathrm{C} 33-\mathrm{C} 38$ & $119.8(4)$ & N7-Fe1-N9 & $79.85(13)$ \\
\hline $\mathrm{C} 34-\mathrm{C} 33-\mathrm{N} 6$ & $118.0(4)$ & N4-Fe1-N9 & $122.57(14)$ \\
\hline $\mathrm{C} 38-\mathrm{C} 33-\mathrm{N} 6$ & $122.3(4)$ & $\mathrm{N} 10-\mathrm{Fe} 1-\mathrm{Fe} 2$ & $143.01(11)$ \\
\hline $\mathrm{C} 35-\mathrm{C} 34-\mathrm{C} 33$ & $122.4(5)$ & $\mathrm{N} 7-\mathrm{Fe} 1-\mathrm{Fe} 2$ & $45.05(10)$ \\
\hline $\mathrm{C} 35-\mathrm{C} 34-\mathrm{H} 34$ & 118.8 & $\mathrm{~N} 4-\mathrm{Fe} 1-\mathrm{Fe} 2$ & $45.18(10)$ \\
\hline $\mathrm{C} 33-\mathrm{C} 34-\mathrm{H} 34$ & 118.8 & N9-Fe1-Fe2 & $108.97(10)$ \\
\hline $\mathrm{C} 34-\mathrm{C} 35-\mathrm{C} 36$ & $117.7(5)$ & $\mathrm{N} 1-\mathrm{Fe} 2-\mathrm{N} 7$ & $123.16(15)$ \\
\hline C $34-\mathrm{C} 35-\mathrm{H} 35$ & 121.1 & $\mathrm{~N} 1-\mathrm{Fe} 2-\mathrm{N} 4$ & $143.61(15)$ \\
\hline $\mathrm{C} 36-\mathrm{C} 35-\mathrm{H} 35$ & 121.1 & $\mathrm{~N} 7-\mathrm{Fe} 2-\mathrm{N} 4$ & $93.04(14)$ \\
\hline $\mathrm{C} 37-\mathrm{C} 36-\mathrm{C} 35$ & $120.9(5)$ & $\mathrm{N} 1-\mathrm{Fe} 2-\mathrm{C} 21$ & $119.49(15)$ \\
\hline $\mathrm{C} 37-\mathrm{C} 36-\mathrm{H} 36$ & 119.6 & $\mathrm{~N} 7-\mathrm{Fe} 2-\mathrm{C} 21$ & $110.96(15)$ \\
\hline $\mathrm{C} 35-\mathrm{C} 36-\mathrm{H} 36$ & 119.6 & $\mathrm{~N} 4-\mathrm{Fe} 2-\mathrm{C} 21$ & $36.19(14)$ \\
\hline $\mathrm{C} 36-\mathrm{C} 37-\mathrm{C} 38$ & $121.8(5)$ & $\mathrm{N} 1-\mathrm{Fe} 2-\mathrm{Fe} 1$ & $168.73(11)$ \\
\hline $\mathrm{C} 36-\mathrm{C} 37-\mathrm{H} 37$ & 119.1 & $\mathrm{~N} 7-\mathrm{Fe} 2-\mathrm{Fe} 1$ & $45.87(10)$ \\
\hline $\mathrm{C} 38-\mathrm{C} 37-\mathrm{H} 37$ & 119.1 & $\mathrm{~N} 4-\mathrm{Fe} 2-\mathrm{Fe} 1$ & $47.62(10)$ \\
\hline $\mathrm{C} 33-\mathrm{C} 38-\mathrm{C} 37$ & $117.4(4)$ & $\mathrm{C} 21-\mathrm{Fe} 2-\mathrm{Fe} 1$ & $68.99(11)$ \\
\hline $\mathrm{C} 33-\mathrm{C} 38-\mathrm{N} 4$ & $126.0(4)$ & $\mathrm{C} 18-\mathrm{N} 1-\mathrm{C} 1$ & $122.3(4)$ \\
\hline $\mathrm{C} 37-\mathrm{C} 38-\mathrm{N} 4$ & $116.3(4)$ & $\mathrm{C} 18-\mathrm{N} 1-\mathrm{Fe} 2$ & $128.7(3)$ \\
\hline $\mathrm{N} 5-\mathrm{C} 39-\mathrm{H} 39 \mathrm{~A}$ & 109.5 & $\mathrm{C} 1-\mathrm{N} 1-\mathrm{Fe} 2$ & $108.3(3)$ \\
\hline N5-C39-H39B & 109.5 & $\mathrm{C} 7-\mathrm{N} 2-\mathrm{C} 6$ & $109.9(4)$ \\
\hline H39A-C39-H39B & 109.5 & $\mathrm{C} 7-\mathrm{N} 2-\mathrm{C} 19$ & $115.6(4)$ \\
\hline $\mathrm{N} 5-\mathrm{C} 39-\mathrm{H} 39 \mathrm{C}$ & 109.5 & $\mathrm{C} 6-\mathrm{N} 2-\mathrm{C} 19$ & $112.0(4)$ \\
\hline $\mathrm{H} 39 \mathrm{~A}-\mathrm{C} 39-\mathrm{H} 39 \mathrm{C}$ & 109.5 & $\mathrm{C} 12-\mathrm{N} 3-\mathrm{C} 13$ & $116.3(4)$ \\
\hline $\mathrm{H} 39 \mathrm{~B}-\mathrm{C} 39-\mathrm{H} 39 \mathrm{C}$ & 109.5 & $\mathrm{C} 12-\mathrm{N} 3-\mathrm{C} 20$ & $118.6(4)$ \\
\hline $\mathrm{N} 6-\mathrm{C} 40-\mathrm{H} 40 \mathrm{~A}$ & 109.5 & $\mathrm{C} 13-\mathrm{N} 3-\mathrm{C} 20$ & $112.2(4)$ \\
\hline $\mathrm{N} 6-\mathrm{C} 40-\mathrm{H} 40 \mathrm{~B}$ & 109.5 & $\mathrm{C} 38-\mathrm{N} 4-\mathrm{C} 21$ & $120.8(3)$ \\
\hline $\mathrm{H} 40 \mathrm{~A}-\mathrm{C} 40-\mathrm{H} 40 \mathrm{~B}$ & 109.5 & $\mathrm{C} 38-\mathrm{N} 4-\mathrm{Fe} 2$ & $130.2(3)$ \\
\hline $\mathrm{N} 6-\mathrm{C} 40-\mathrm{H} 40 \mathrm{C}$ & 109.5 & $\mathrm{C} 21-\mathrm{N} 4-\mathrm{Fe} 2$ & $85.6(2)$ \\
\hline $\mathrm{H} 40 \mathrm{~A}-\mathrm{C} 40-\mathrm{H} 40 \mathrm{C}$ & 109.5 & $\mathrm{C} 38-\mathrm{N} 4-\mathrm{Fe} 1$ & $112.6(3)$ \\
\hline $\mathrm{H} 40 \mathrm{~B}-\mathrm{C} 40-\mathrm{H} 40 \mathrm{C}$ & 109.5 & $\mathrm{C} 21-\mathrm{N} 4-\mathrm{Fe} 1$ & $114.8(3)$ \\
\hline $\mathrm{C} 42-\mathrm{C} 41-\mathrm{C} 46$ & $118.2(4)$ & $\mathrm{Fe} 2-\mathrm{N} 4-\mathrm{Fe} 1$ & $87.20(13)$ \\
\hline $\mathrm{C} 42-\mathrm{C} 41-\mathrm{N} 7$ & $120.6(4)$ & $\mathrm{C} 27-\mathrm{N} 5-\mathrm{C} 26$ & $113.2(4)$ \\
\hline $\mathrm{C} 46-\mathrm{C} 41-\mathrm{N} 7$ & $121.1(4)$ & $\mathrm{C} 27-\mathrm{N} 5-\mathrm{C} 39$ & $117.9(4)$ \\
\hline $\mathrm{C} 43-\mathrm{C} 42-\mathrm{C} 41$ & $122.0(5)$ & $\mathrm{C} 26-\mathrm{N} 5-\mathrm{C} 39$ & $113.7(4)$ \\
\hline $\mathrm{C} 43-\mathrm{C} 42-\mathrm{H} 42$ & 119.0 & $\mathrm{C} 32-\mathrm{N} 6-\mathrm{C} 33$ & $112.0(4)$ \\
\hline $\mathrm{C} 41-\mathrm{C} 42-\mathrm{H} 42$ & 119.0 & $\mathrm{C} 32-\mathrm{N} 6-\mathrm{C} 40$ & $116.6(4)$ \\
\hline $\mathrm{C} 42-\mathrm{C} 43-\mathrm{C} 44$ & $120.0(5)$ & $\mathrm{C} 33-\mathrm{N} 6-\mathrm{C} 40$ & $114.5(4)$ \\
\hline $\mathrm{C} 42-\mathrm{C} 43-\mathrm{H} 43$ & 120.0 & $\mathrm{C} 41-\mathrm{N} 7-\mathrm{C} 58$ & $113.5(3)$ \\
\hline $\mathrm{C} 44-\mathrm{C} 43-\mathrm{H} 43$ & 120.0 & $\mathrm{C} 41-\mathrm{N} 7-\mathrm{Fe} 2$ & $120.7(3)$ \\
\hline $\mathrm{C} 43-\mathrm{C} 44-\mathrm{C} 45$ & $119.1(5)$ & $\mathrm{C} 58-\mathrm{N} 7-\mathrm{Fe} 2$ & $98.9(3)$ \\
\hline $\mathrm{C} 43-\mathrm{C} 44-\mathrm{H} 44$ & 120.4 & $\mathrm{C} 41-\mathrm{N} 7-\mathrm{Fe} 1$ & $123.5(3)$ \\
\hline $\mathrm{C} 45-\mathrm{C} 44-\mathrm{H} 44$ & 120.4 & $\mathrm{C} 58-\mathrm{N} 7-\mathrm{Fe} 1$ & $106.5(3)$ \\
\hline $\mathrm{C} 46-\mathrm{C} 45-\mathrm{C} 44$ & $121.7(5)$ & $\mathrm{Fe} 2-\mathrm{N} 7-\mathrm{Fe} 1$ & 89.07 (13) \\
\hline $\mathrm{C} 46-\mathrm{C} 45-\mathrm{H} 45$ & 119.1 & $\mathrm{C} 46-\mathrm{N} 8-\mathrm{C} 47$ & $115.1(4)$ \\
\hline
\end{tabular}




\begin{tabular}{|c|c|c|c|}
\hline $\mathrm{C} 44-\mathrm{C} 45-\mathrm{H} 45$ & 119.1 & $\mathrm{C} 46-\mathrm{N} 8-\mathrm{C} 59$ & $116.8(4)$ \\
\hline $\mathrm{C} 45-\mathrm{C} 46-\mathrm{C} 41$ & $118.7(5)$ & $\mathrm{C} 47-\mathrm{N} 8-\mathrm{C} 59$ & $114.4(4)$ \\
\hline $\mathrm{C} 45-\mathrm{C} 46-\mathrm{N} 8$ & $123.3(4)$ & $\mathrm{C} 53-\mathrm{N} 9-\mathrm{C} 52$ & $114.4(3)$ \\
\hline $\mathrm{C} 41-\mathrm{C} 46-\mathrm{N} 8$ & $118.0(4)$ & $\mathrm{C} 53-\mathrm{N} 9-\mathrm{C} 60$ & $111.7(4)$ \\
\hline $\mathrm{C} 48-\mathrm{C} 47-\mathrm{C} 52$ & $119.2(5)$ & $\mathrm{C} 52-\mathrm{N} 9-\mathrm{C} 60$ & $112.1(4)$ \\
\hline $\mathrm{C} 48-\mathrm{C} 47-\mathrm{N} 8$ & $116.8(4)$ & $\mathrm{C} 53-\mathrm{N} 9-\mathrm{Fe} 1$ & $102.3(3)$ \\
\hline $\mathrm{C} 52-\mathrm{C} 47-\mathrm{N} 8$ & $124.1(4)$ & $\mathrm{C} 52-\mathrm{N} 9-\mathrm{Fe} 1$ & $104.0(3)$ \\
\hline $\mathrm{C} 49-\mathrm{C} 48-\mathrm{C} 47$ & $122.9(5)$ & $\mathrm{C} 60-\mathrm{N} 9-\mathrm{Fe} 1$ & $111.6(3)$ \\
\hline $\mathrm{C} 49-\mathrm{C} 48-\mathrm{H} 48$ & 118.6 & $\mathrm{C} 78-\mathrm{N} 10-\mathrm{C} 61$ & $123.3(3)$ \\
\hline $\mathrm{C} 47-\mathrm{C} 48-\mathrm{H} 48$ & 118.6 & $\mathrm{C} 78-\mathrm{N} 10-\mathrm{Fe} 1$ & $122.2(3)$ \\
\hline $\mathrm{C} 50-\mathrm{C} 49-\mathrm{C} 48$ & $117.8(5)$ & $\mathrm{C} 61-\mathrm{N} 10-\mathrm{Fe} 1$ & $114.2(3)$ \\
\hline $\mathrm{C} 50-\mathrm{C} 49-\mathrm{H} 49$ & 121.1 & C67-N11-C66 & $112.4(3)$ \\
\hline $\mathrm{C} 48-\mathrm{C} 49-\mathrm{H} 49$ & 121.1 & C67-N11-C79 & $117.2(3)$ \\
\hline $\mathrm{C} 49-\mathrm{C} 50-\mathrm{C} 51$ & $121.0(5)$ & $\mathrm{C} 66-\mathrm{N} 11-\mathrm{C} 79$ & $112.6(3)$ \\
\hline $\mathrm{C} 49-\mathrm{C} 50-\mathrm{H} 50$ & 119.5 & $\mathrm{C} 72-\mathrm{N} 12-\mathrm{C} 73$ & $113.7(3)$ \\
\hline $\mathrm{C} 51-\mathrm{C} 50-\mathrm{H} 50$ & 119.5 & $\mathrm{C} 72-\mathrm{N} 12-\mathrm{C} 80$ & $117.2(4)$ \\
\hline $\mathrm{C} 50-\mathrm{C} 51-\mathrm{C} 52$ & $121.1(5)$ & $\mathrm{C} 73-\mathrm{N} 12-\mathrm{C} 80$ & $113.6(4)$ \\
\hline $\mathrm{C} 6-\mathrm{C} 1-\mathrm{C} 2-\mathrm{C} 3$ & $-2.4(7)$ & $\mathrm{N} 11-\mathrm{C} 67-\mathrm{C} 72-\mathrm{N} 12$ & $-3.9(6)$ \\
\hline $\mathrm{N} 1-\mathrm{C} 1-\mathrm{C} 2-\mathrm{C} 3$ & $171.3(4)$ & $\mathrm{C} 78-\mathrm{C} 73-\mathrm{C} 74-\mathrm{C} 75$ & $-0.7(7)$ \\
\hline $\mathrm{C} 1-\mathrm{C} 2-\mathrm{C} 3-\mathrm{C} 4$ & $0.4(8)$ & $\mathrm{N} 12-\mathrm{C} 73-\mathrm{C} 74-\mathrm{C} 75$ & $-179.2(4)$ \\
\hline $\mathrm{C} 2-\mathrm{C} 3-\mathrm{C} 4-\mathrm{C} 5$ & $2.1(9)$ & $\mathrm{C} 73-\mathrm{C} 74-\mathrm{C} 75-\mathrm{C} 76$ & $-0.1(7)$ \\
\hline $\mathrm{C} 3-\mathrm{C} 4-\mathrm{C} 5-\mathrm{C} 6$ & $-2.6(9)$ & $\mathrm{C} 74-\mathrm{C} 75-\mathrm{C} 76-\mathrm{C} 77$ & $0.6(7)$ \\
\hline $\mathrm{C} 4-\mathrm{C} 5-\mathrm{C} 6-\mathrm{C} 1$ & $0.6(8)$ & $\mathrm{C} 75-\mathrm{C} 76-\mathrm{C} 77-\mathrm{C} 78$ & $-0.4(7)$ \\
\hline $\mathrm{C} 4-\mathrm{C} 5-\mathrm{C} 6-\mathrm{N} 2$ & $-180.0(5)$ & $\mathrm{C} 74-\mathrm{C} 73-\mathrm{C} 78-\mathrm{N} 10$ & $-177.4(4)$ \\
\hline $\mathrm{C} 2-\mathrm{C} 1-\mathrm{C} 6-\mathrm{C} 5$ & $1.9(7)$ & $\mathrm{N} 12-\mathrm{C} 73-\mathrm{C} 78-\mathrm{N} 10$ & $1.2(6)$ \\
\hline $\mathrm{N} 1-\mathrm{C} 1-\mathrm{C} 6-\mathrm{C} 5$ & $-171.3(5)$ & $\mathrm{C} 74-\mathrm{C} 73-\mathrm{C} 78-\mathrm{C} 77$ & $0.8(6)$ \\
\hline $\mathrm{C} 2-\mathrm{C} 1-\mathrm{C} 6-\mathrm{N} 2$ & $-177.6(4)$ & $\mathrm{N} 12-\mathrm{C} 73-\mathrm{C} 78-\mathrm{C} 77$ & $179.4(4)$ \\
\hline $\mathrm{N} 1-\mathrm{C} 1-\mathrm{C} 6-\mathrm{N} 2$ & $9.2(8)$ & $\mathrm{C} 76-\mathrm{C} 77-\mathrm{C} 78-\mathrm{N} 10$ & $178.0(4)$ \\
\hline $\mathrm{C} 12-\mathrm{C} 7-\mathrm{C} 8-\mathrm{C} 9$ & $4.0(7)$ & $\mathrm{C} 76-\mathrm{C} 77-\mathrm{C} 78-\mathrm{C} 73$ & $-0.4(6)$ \\
\hline $\mathrm{N} 2-\mathrm{C} 7-\mathrm{C} 8-\mathrm{C} 9$ & $-174.4(4)$ & $\mathrm{C} 17-\mathrm{C} 18-\mathrm{N} 1-\mathrm{C} 1$ & $173.8(4)$ \\
\hline $\mathrm{C} 7-\mathrm{C} 8-\mathrm{C} 9-\mathrm{C} 10$ & $2.1(8)$ & $\mathrm{C} 13-\mathrm{C} 18-\mathrm{N} 1-\mathrm{C} 1$ & $-10.1(7)$ \\
\hline $\mathrm{C} 8-\mathrm{C} 9-\mathrm{C} 10-\mathrm{C} 11$ & $-5.5(8)$ & $\mathrm{C} 17-\mathrm{C} 18-\mathrm{N} 1-\mathrm{Fe} 2$ & $4.8(6)$ \\
\hline $\mathrm{C} 9-\mathrm{C} 10-\mathrm{C} 11-\mathrm{C} 12$ & $2.8(8)$ & $\mathrm{C} 13-\mathrm{C} 18-\mathrm{N} 1-\mathrm{Fe} 2$ & $-179.1(3)$ \\
\hline $\mathrm{C} 10-\mathrm{C} 11-\mathrm{C} 12-\mathrm{N} 3$ & $-179.3(5)$ & $\mathrm{C} 6-\mathrm{C} 1-\mathrm{N} 1-\mathrm{C} 18$ & $-54.7(7)$ \\
\hline $\mathrm{C} 10-\mathrm{C} 11-\mathrm{C} 12-\mathrm{C} 7$ & $3.2(7)$ & $\mathrm{C} 2-\mathrm{C} 1-\mathrm{N} 1-\mathrm{C} 18$ & $132.1(5)$ \\
\hline $\mathrm{C} 8-\mathrm{C} 7-\mathrm{C} 12-\mathrm{N} 3$ & $175.8(4)$ & $\mathrm{C} 6-\mathrm{C} 1-\mathrm{N} 1-\mathrm{Fe} 2$ & $116.3(5)$ \\
\hline $\mathrm{N} 2-\mathrm{C} 7-\mathrm{C} 12-\mathrm{N} 3$ & $-5.6(6)$ & $\mathrm{C} 2-\mathrm{C} 1-\mathrm{N} 1-\mathrm{Fe} 2$ & $-56.9(5)$ \\
\hline $\mathrm{C} 8-\mathrm{C} 7-\mathrm{C} 12-\mathrm{C} 11$ & $-6.6(7)$ & $\mathrm{C} 8-\mathrm{C} 7-\mathrm{N} 2-\mathrm{C} 6$ & $121.6(5)$ \\
\hline $\mathrm{N} 2-\mathrm{C} 7-\mathrm{C} 12-\mathrm{C} 11$ & $171.9(4)$ & $\mathrm{C} 12-\mathrm{C} 7-\mathrm{N} 2-\mathrm{C} 6$ & $-56.9(5)$ \\
\hline $\mathrm{C} 18-\mathrm{C} 13-\mathrm{C} 14-\mathrm{C} 15$ & $3.2(7)$ & $\mathrm{C} 8-\mathrm{C} 7-\mathrm{N} 2-\mathrm{C} 19$ & $-6.4(6)$ \\
\hline $\mathrm{N} 3-\mathrm{C} 13-\mathrm{C} 14-\mathrm{C} 15$ & $-173.0(4)$ & $\mathrm{C} 12-\mathrm{C} 7-\mathrm{N} 2-\mathrm{C} 19$ & $175.1(4)$ \\
\hline $\mathrm{C} 13-\mathrm{C} 14-\mathrm{C} 15-\mathrm{C} 16$ & $0.4(7)$ & $\mathrm{C} 5-\mathrm{C} 6-\mathrm{N} 2-\mathrm{C} 7$ & $-63.7(6)$ \\
\hline $\mathrm{C} 14-\mathrm{C} 15-\mathrm{C} 16-\mathrm{C} 17$ & $-1.8(7)$ & $\mathrm{C} 1-\mathrm{C} 6-\mathrm{N} 2-\mathrm{C} 7$ & $115.8(5)$ \\
\hline $\mathrm{C} 15-\mathrm{C} 16-\mathrm{C} 17-\mathrm{C} 18$ & $-0.4(7)$ & $\mathrm{C} 5-\mathrm{C} 6-\mathrm{N} 2-\mathrm{C} 19$ & $66.3(6)$ \\
\hline $\mathrm{C} 16-\mathrm{C} 17-\mathrm{C} 18-\mathrm{N} 1$ & $-179.6(4)$ & $\mathrm{C} 1-\mathrm{C} 6-\mathrm{N} 2-\mathrm{C} 19$ & $-114.2(5)$ \\
\hline $\mathrm{C} 16-\mathrm{C} 17-\mathrm{C} 18-\mathrm{C} 13$ & $3.9(7)$ & $\mathrm{C} 11-\mathrm{C} 12-\mathrm{N} 3-\mathrm{C} 13$ & $118.5(5)$ \\
\hline $\mathrm{C} 14-\mathrm{C} 13-\mathrm{C} 18-\mathrm{N} 1$ & $178.6(4)$ & $\mathrm{C} 7-\mathrm{C} 12-\mathrm{N} 3-\mathrm{C} 13$ & $-64.1(6)$ \\
\hline
\end{tabular}




\begin{tabular}{|c|c|}
\hline $\mathrm{N} 3-\mathrm{C} 13-\mathrm{C} 18-\mathrm{N} 1$ & $-5.3(7)$ \\
\hline $\mathrm{C} 14-\mathrm{C} 13-\mathrm{C} 18-\mathrm{C} 17$ & $-5.2(7)$ \\
\hline $\mathrm{N} 3-\mathrm{C} 13-\mathrm{C} 18-\mathrm{C} 17$ & $170.9(4)$ \\
\hline $\mathrm{C} 26-\mathrm{C} 21-\mathrm{C} 22-\mathrm{C} 23$ & $2.2(6)$ \\
\hline $\mathrm{N} 4-\mathrm{C} 21-\mathrm{C} 22-\mathrm{C} 23$ & $179.0(4)$ \\
\hline $\mathrm{Fe} 2-\mathrm{C} 21-\mathrm{C} 22-\mathrm{C} 23$ & $121.9(4)$ \\
\hline $\mathrm{C} 21-\mathrm{C} 22-\mathrm{C} 23-\mathrm{C} 24$ & $0.5(6)$ \\
\hline $\mathrm{C} 22-\mathrm{C} 23-\mathrm{C} 24-\mathrm{C} 25$ & $-2.7(6)$ \\
\hline $\mathrm{C} 23-\mathrm{C} 24-\mathrm{C} 25-\mathrm{C} 26$ & $2.2(7)$ \\
\hline $\mathrm{C} 24-\mathrm{C} 25-\mathrm{C} 26-\mathrm{C} 21$ & $0.5(7)$ \\
\hline $\mathrm{C} 24-\mathrm{C} 25-\mathrm{C} 26-\mathrm{N} 5$ & $-179.3(4)$ \\
\hline $\mathrm{C} 22-\mathrm{C} 21-\mathrm{C} 26-\mathrm{C} 25$ & $-2.7(6)$ \\
\hline $\mathrm{N} 4-\mathrm{C} 21-\mathrm{C} 26-\mathrm{C} 25$ & $-179.3(4)$ \\
\hline $\mathrm{Fe} 2-\mathrm{C} 21-\mathrm{C} 26-\mathrm{C} 25$ & $-113.1(4)$ \\
\hline $\mathrm{C} 22-\mathrm{C} 21-\mathrm{C} 26-\mathrm{N} 5$ & $177.1(4)$ \\
\hline $\mathrm{N} 4-\mathrm{C} 21-\mathrm{C} 26-\mathrm{N} 5$ & $0.5(6)$ \\
\hline $\mathrm{Fe} 2-\mathrm{C} 21-\mathrm{C} 26-\mathrm{N} 5$ & $66.7(5)$ \\
\hline $\mathrm{N} 5-\mathrm{C} 27-\mathrm{C} 28-\mathrm{C} 29$ & $-175.5(5)$ \\
\hline $\mathrm{C} 32-\mathrm{C} 27-\mathrm{C} 28-\mathrm{C} 29$ & $3.5(8)$ \\
\hline $\mathrm{C} 27-\mathrm{C} 28-\mathrm{C} 29-\mathrm{C} 30$ & $1.1(10)$ \\
\hline $\mathrm{C} 28-\mathrm{C} 29-\mathrm{C} 30-\mathrm{C} 31$ & $-3.6(10)$ \\
\hline $\mathrm{C} 29-\mathrm{C} 30-\mathrm{C} 31-\mathrm{C} 32$ & $1.3(9)$ \\
\hline $\mathrm{C} 30-\mathrm{C} 31-\mathrm{C} 32-\mathrm{C} 27$ & $3.3(8)$ \\
\hline $\mathrm{C} 30-\mathrm{C} 31-\mathrm{C} 32-\mathrm{N} 6$ & $-174.6(5)$ \\
\hline $\mathrm{C} 28-\mathrm{C} 27-\mathrm{C} 32-\mathrm{C} 31$ & $-5.7(7)$ \\
\hline $\mathrm{N} 5-\mathrm{C} 27-\mathrm{C} 32-\mathrm{C} 31$ & $173.4(4)$ \\
\hline $\mathrm{C} 28-\mathrm{C} 27-\mathrm{C} 32-\mathrm{N} 6$ & $172.5(4)$ \\
\hline $\mathrm{N} 5-\mathrm{C} 27-\mathrm{C} 32-\mathrm{N} 6$ & $-8.5(6)$ \\
\hline $\mathrm{C} 38-\mathrm{C} 33-\mathrm{C} 34-\mathrm{C} 35$ & $-0.1(8)$ \\
\hline $\mathrm{N} 6-\mathrm{C} 33-\mathrm{C} 34-\mathrm{C} 35$ & $179.3(5)$ \\
\hline $\mathrm{C} 33-\mathrm{C} 34-\mathrm{C} 35-\mathrm{C} 36$ & $-1.2(9)$ \\
\hline $\mathrm{C} 34-\mathrm{C} 35-\mathrm{C} 36-\mathrm{C} 37$ & $-0.2(10)$ \\
\hline $\mathrm{C} 35-\mathrm{C} 36-\mathrm{C} 37-\mathrm{C} 38$ & $2.7(9)$ \\
\hline $\mathrm{C} 34-\mathrm{C} 33-\mathrm{C} 38-\mathrm{C} 37$ & $2.5(7)$ \\
\hline $\mathrm{N} 6-\mathrm{C} 33-\mathrm{C} 38-\mathrm{C} 37$ & $-176.8(4)$ \\
\hline $\mathrm{C} 34-\mathrm{C} 33-\mathrm{C} 38-\mathrm{N} 4$ & $-171.1(5)$ \\
\hline $\mathrm{N} 6-\mathrm{C} 33-\mathrm{C} 38-\mathrm{N} 4$ & $9.6(7)$ \\
\hline $\mathrm{C} 36-\mathrm{C} 37-\mathrm{C} 38-\mathrm{C} 33$ & $-3.9(8)$ \\
\hline $\mathrm{C} 36-\mathrm{C} 37-\mathrm{C} 38-\mathrm{N} 4$ & $170.3(5)$ \\
\hline $\mathrm{C} 46-\mathrm{C} 41-\mathrm{C} 42-\mathrm{C} 43$ & $2.5(7)$ \\
\hline $\mathrm{N} 7-\mathrm{C} 41-\mathrm{C} 42-\mathrm{C} 43$ & $-178.2(4)$ \\
\hline $\mathrm{C} 41-\mathrm{C} 42-\mathrm{C} 43-\mathrm{C} 44$ & $1.0(8)$ \\
\hline $\mathrm{C} 42-\mathrm{C} 43-\mathrm{C} 44-\mathrm{C} 45$ & $-2.2(8)$ \\
\hline $\mathrm{C} 43-\mathrm{C} 44-\mathrm{C} 45-\mathrm{C} 46$ & $-0.1(9)$ \\
\hline $\mathrm{C} 44-\mathrm{C} 45-\mathrm{C} 46-\mathrm{C} 41$ & $3.6(8)$ \\
\hline $\mathrm{C} 44-\mathrm{C} 45-\mathrm{C} 46-\mathrm{N} 8$ & $-175.8(5)$ \\
\hline $\mathrm{C} 42-\mathrm{C} 41-\mathrm{C} 46-\mathrm{C} 45$ & $-4.7(7)$ \\
\hline $\mathrm{N} 7-\mathrm{C} 41-\mathrm{C} 46-\mathrm{C} 45$ & $176.0(4)$ \\
\hline
\end{tabular}

\begin{tabular}{|c|c|}
\hline $\mathrm{C} 11-\mathrm{C} 12-\mathrm{N} 3-\mathrm{C} 20$ & $-20.1(7)$ \\
\hline $\mathrm{C} 7-\mathrm{C} 12-\mathrm{N} 3-\mathrm{C} 20$ & $157.4(4)$ \\
\hline $\mathrm{C} 14-\mathrm{C} 13-\mathrm{N} 3-\mathrm{C} 12$ & $-65.1(6)$ \\
\hline $\mathrm{C} 18-\mathrm{C} 13-\mathrm{N} 3-\mathrm{C} 12$ & $118.7(5)$ \\
\hline $\mathrm{C} 14-\mathrm{C} 13-\mathrm{N} 3-\mathrm{C} 20$ & $76.0(5)$ \\
\hline $\mathrm{C} 18-\mathrm{C} 13-\mathrm{N} 3-\mathrm{C} 20$ & $-100.2(5)$ \\
\hline $\mathrm{C} 33-\mathrm{C} 38-\mathrm{N} 4-\mathrm{C} 21$ & $-30.0(7)$ \\
\hline $\mathrm{C} 37-\mathrm{C} 38-\mathrm{N} 4-\mathrm{C} 21$ & $156.3(4)$ \\
\hline $\mathrm{C} 33-\mathrm{C} 38-\mathrm{N} 4-\mathrm{Fe} 2$ & $-142.7(4)$ \\
\hline $\mathrm{C} 37-\mathrm{C} 38-\mathrm{N} 4-\mathrm{Fe} 2$ & $43.7(6)$ \\
\hline $\mathrm{C} 33-\mathrm{C} 38-\mathrm{N} 4-\mathrm{Fe} 1$ & $110.9(5)$ \\
\hline $\mathrm{C} 37-\mathrm{C} 38-\mathrm{N} 4-\mathrm{Fe} 1$ & $-62.7(5)$ \\
\hline $\mathrm{C} 22-\mathrm{C} 21-\mathrm{N} 4-\mathrm{C} 38$ & $145.9(4)$ \\
\hline $\mathrm{C} 26-\mathrm{C} 21-\mathrm{N} 4-\mathrm{C} 38$ & $-37.5(6)$ \\
\hline $\mathrm{Fe} 2-\mathrm{C} 21-\mathrm{N} 4-\mathrm{C} 38$ & $-135.0(4)$ \\
\hline $\mathrm{C} 22-\mathrm{C} 21-\mathrm{N} 4-\mathrm{Fe} 2$ & $-79.1(4)$ \\
\hline $\mathrm{C} 26-\mathrm{C} 21-\mathrm{N} 4-\mathrm{Fe} 2$ & $97.5(4)$ \\
\hline $\mathrm{C} 22-\mathrm{C} 21-\mathrm{N} 4-\mathrm{Fe} 1$ & $5.8(5)$ \\
\hline $\mathrm{C} 26-\mathrm{C} 21-\mathrm{N} 4-\mathrm{Fe} 1$ & $-177.7(3)$ \\
\hline $\mathrm{Fe} 2-\mathrm{C} 21-\mathrm{N} 4-\mathrm{Fe} 1$ & $84.8(2)$ \\
\hline $\mathrm{C} 28-\mathrm{C} 27-\mathrm{N} 5-\mathrm{C} 26$ & $124.2(5)$ \\
\hline $\mathrm{C} 32-\mathrm{C} 27-\mathrm{N} 5-\mathrm{C} 26$ & $-54.8(6)$ \\
\hline $\mathrm{C} 28-\mathrm{C} 27-\mathrm{N} 5-\mathrm{C} 39$ & $-12.1(7)$ \\
\hline $\mathrm{C} 32-\mathrm{C} 27-\mathrm{N} 5-\mathrm{C} 39$ & $168.9(4)$ \\
\hline $\mathrm{C} 25-\mathrm{C} 26-\mathrm{N} 5-\mathrm{C} 27$ & $-59.5(6)$ \\
\hline $\mathrm{C} 21-\mathrm{C} 26-\mathrm{N} 5-\mathrm{C} 27$ & $120.7(4)$ \\
\hline $\mathrm{C} 25-\mathrm{C} 26-\mathrm{N} 5-\mathrm{C} 39$ & $78.7(5)$ \\
\hline $\mathrm{C} 21-\mathrm{C} 26-\mathrm{N} 5-\mathrm{C} 39$ & $-101.1(5)$ \\
\hline $\mathrm{C} 31-\mathrm{C} 32-\mathrm{N} 6-\mathrm{C} 33$ & $116.0(5)$ \\
\hline $\mathrm{C} 27-\mathrm{C} 32-\mathrm{N} 6-\mathrm{C} 33$ & $-62.0(5)$ \\
\hline $\mathrm{C} 31-\mathrm{C} 32-\mathrm{N} 6-\mathrm{C} 40$ & $-18.5(7)$ \\
\hline $\mathrm{C} 27-\mathrm{C} 32-\mathrm{N} 6-\mathrm{C} 40$ & $163.5(4)$ \\
\hline $\mathrm{C} 34-\mathrm{C} 33-\mathrm{N} 6-\mathrm{C} 32$ & $-66.3(6)$ \\
\hline $\mathrm{C} 38-\mathrm{C} 33-\mathrm{N} 6-\mathrm{C} 32$ & $113.1(5)$ \\
\hline $\mathrm{C} 34-\mathrm{C} 33-\mathrm{N} 6-\mathrm{C} 40$ & $69.3(6)$ \\
\hline $\mathrm{C} 38-\mathrm{C} 33-\mathrm{N} 6-\mathrm{C} 40$ & $-111.4(5)$ \\
\hline $\mathrm{C} 42-\mathrm{C} 41-\mathrm{N} 7-\mathrm{C} 58$ & $144.3(4)$ \\
\hline $\mathrm{C} 46-\mathrm{C} 41-\mathrm{N} 7-\mathrm{C} 58$ & $-36.4(6)$ \\
\hline $\mathrm{C} 42-\mathrm{C} 41-\mathrm{N} 7-\mathrm{Fe} 2$ & $27.3(5)$ \\
\hline $\mathrm{C} 46-\mathrm{C} 41-\mathrm{N} 7-\mathrm{Fe} 2$ & $-153.4(3)$ \\
\hline $\mathrm{C} 42-\mathrm{C} 41-\mathrm{N} 7-\mathrm{Fe} 1$ & $-84.5(5)$ \\
\hline $\mathrm{C} 46-\mathrm{C} 41-\mathrm{N} 7-\mathrm{Fe} 1$ & $94.8(4)$ \\
\hline $\mathrm{C} 57-\mathrm{C} 58-\mathrm{N} 7-\mathrm{C} 41$ & $-67.4(6)$ \\
\hline $\mathrm{C} 53-\mathrm{C} 58-\mathrm{N} 7-\mathrm{C} 41$ & $111.8(5)$ \\
\hline $\mathrm{C} 57-\mathrm{C} 58-\mathrm{N} 7-\mathrm{Fe} 2$ & $61.8(4)$ \\
\hline $\mathrm{C} 53-\mathrm{C} 58-\mathrm{N} 7-\mathrm{Fe} 2$ & $-119.0(4)$ \\
\hline $\mathrm{C} 57-\mathrm{C} 58-\mathrm{N} 7-\mathrm{Fe} 1$ & $153.5(4)$ \\
\hline $\mathrm{C} 53-\mathrm{C} 58-\mathrm{N} 7-\mathrm{Fe} 1$ & $-27.3(5)$ \\
\hline
\end{tabular}




\begin{tabular}{|c|c|}
\hline $\mathrm{C} 42-\mathrm{C} 41-\mathrm{C} 46-\mathrm{N} 8$ & $174.7(4)$ \\
\hline $\mathrm{N} 7-\mathrm{C} 41-\mathrm{C} 46-\mathrm{N} 8$ & $-4.6(6)$ \\
\hline $\mathrm{C} 52-\mathrm{C} 47-\mathrm{C} 48-\mathrm{C} 49$ & $0.5(8)$ \\
\hline $\mathrm{N} 8-\mathrm{C} 47-\mathrm{C} 48-\mathrm{C} 49$ & $178.8(5)$ \\
\hline $\mathrm{C} 47-\mathrm{C} 48-\mathrm{C} 49-\mathrm{C} 50$ & $-1.3(8)$ \\
\hline $\mathrm{C} 48-\mathrm{C} 49-\mathrm{C} 50-\mathrm{C} 51$ & $0.1(8)$ \\
\hline $\mathrm{C} 49-\mathrm{C} 50-\mathrm{C} 51-\mathrm{C} 52$ & $1.8(8)$ \\
\hline $\mathrm{C} 48-\mathrm{C} 47-\mathrm{C} 52-\mathrm{C} 51$ & $1.4(7)$ \\
\hline $\mathrm{N} 8-\mathrm{C} 47-\mathrm{C} 52-\mathrm{C} 51$ & $-176.8(4)$ \\
\hline $\mathrm{C} 48-\mathrm{C} 47-\mathrm{C} 52-\mathrm{N} 9$ & $179.1(4)$ \\
\hline $\mathrm{N} 8-\mathrm{C} 47-\mathrm{C} 52-\mathrm{N} 9$ & $0.8(7)$ \\
\hline $\mathrm{C} 50-\mathrm{C} 51-\mathrm{C} 52-\mathrm{C} 47$ & $-2.6(7)$ \\
\hline $\mathrm{C} 50-\mathrm{C} 51-\mathrm{C} 52-\mathrm{N} 9$ & $179.6(4)$ \\
\hline $\mathrm{C} 58-\mathrm{C} 53-\mathrm{C} 54-\mathrm{C} 55$ & $0.9(7)$ \\
\hline N9-C53-C54-C55 & $-178.4(4)$ \\
\hline $\mathrm{C} 53-\mathrm{C} 54-\mathrm{C} 55-\mathrm{C} 56$ & $-0.1(8)$ \\
\hline $\mathrm{C} 54-\mathrm{C} 55-\mathrm{C} 56-\mathrm{C} 57$ & $-0.6(8)$ \\
\hline $\mathrm{C} 55-\mathrm{C} 56-\mathrm{C} 57-\mathrm{C} 58$ & $0.3(8)$ \\
\hline $\mathrm{C} 56-\mathrm{C} 57-\mathrm{C} 58-\mathrm{C} 53$ & $0.5(7)$ \\
\hline $\mathrm{C} 56-\mathrm{C} 57-\mathrm{C} 58-\mathrm{N} 7$ & $179.7(4)$ \\
\hline $\mathrm{C} 54-\mathrm{C} 53-\mathrm{C} 58-\mathrm{C} 57$ & $-1.1(7)$ \\
\hline N9-C53-C58-C57 & $178.2(4)$ \\
\hline $\mathrm{C} 54-\mathrm{C} 53-\mathrm{C} 58-\mathrm{N} 7$ & $179.6(4)$ \\
\hline $\mathrm{N} 9-\mathrm{C} 53-\mathrm{C} 58-\mathrm{N} 7$ & $-1.0(6)$ \\
\hline $\mathrm{N} 10-\mathrm{C} 61-\mathrm{C} 62-\mathrm{C} 63$ & $169.3(4)$ \\
\hline $\mathrm{C} 66-\mathrm{C} 61-\mathrm{C} 62-\mathrm{C} 63$ & $-3.2(7)$ \\
\hline $\mathrm{C} 61-\mathrm{C} 62-\mathrm{C} 63-\mathrm{C} 64$ & $2.0(7)$ \\
\hline C62-C63-C64-C65 & $0.8(7)$ \\
\hline $\mathrm{C} 63-\mathrm{C} 64-\mathrm{C} 65-\mathrm{C} 66$ & $-2.2(7)$ \\
\hline $\mathrm{C} 64-\mathrm{C} 65-\mathrm{C} 66-\mathrm{C} 61$ & $0.9(7)$ \\
\hline $\mathrm{C} 64-\mathrm{C} 65-\mathrm{C} 66-\mathrm{N} 11$ & $-179.4(4)$ \\
\hline $\mathrm{N} 10-\mathrm{C} 61-\mathrm{C} 66-\mathrm{C} 65$ & $-170.3(4)$ \\
\hline $\mathrm{C} 62-\mathrm{C} 61-\mathrm{C} 66-\mathrm{C} 65$ & $1.7(6)$ \\
\hline $\mathrm{N} 10-\mathrm{C} 61-\mathrm{C} 66-\mathrm{N} 11$ & $10.0(7)$ \\
\hline $\mathrm{C} 62-\mathrm{C} 61-\mathrm{C} 66-\mathrm{N} 11$ & $-178.0(4)$ \\
\hline $\mathrm{C} 72-\mathrm{C} 67-\mathrm{C} 68-\mathrm{C} 69$ & $2.5(7)$ \\
\hline $\mathrm{N} 11-\mathrm{C} 67-\mathrm{C} 68-\mathrm{C} 69$ & $-176.9(4)$ \\
\hline $\mathrm{C} 67-\mathrm{C} 68-\mathrm{C} 69-\mathrm{C} 70$ & $-0.2(8)$ \\
\hline $\mathrm{C} 68-\mathrm{C} 69-\mathrm{C} 70-\mathrm{C} 71$ & $-2.1(8)$ \\
\hline $\mathrm{C} 69-\mathrm{C} 70-\mathrm{C} 71-\mathrm{C} 72$ & $2.2(7)$ \\
\hline $\mathrm{C} 70-\mathrm{C} 71-\mathrm{C} 72-\mathrm{C} 67$ & $0.0(7)$ \\
\hline $\mathrm{C} 70-\mathrm{C} 71-\mathrm{C} 72-\mathrm{N} 12$ & $-178.9(4)$ \\
\hline $\mathrm{C} 68-\mathrm{C} 67-\mathrm{C} 72-\mathrm{C} 71$ & $-2.4(7)$ \\
\hline $\mathrm{N} 11-\mathrm{C} 67-\mathrm{C} 72-\mathrm{C} 71$ & $177.1(4)$ \\
\hline $\mathrm{C} 68-\mathrm{C} 67-\mathrm{C} 72-\mathrm{N} 12$ & $176.6(4)$ \\
\hline
\end{tabular}

$95.8(5)$

$-83.6(5)$

$-42.5(7)$

$138.1(4)$

$-77.2(5)$

$101.1(5)$

$62.1(6)$

$-119.6(5)$

$93.7(5)$

$-85.6(5)$

$-34.9(6)$

$145.8(4)$

$-154.5(4)$

$26.2(4)$

$21.8(6)$

$-160.5(4)$

$150.3(4)$

$-32.1(5)$

$-89.0(4)$

88.7 (4)

$-23.2(6)$

$158.6(4)$

$163.5(3)$

$-14.7(5)$

$142.7(4)$

$-45.4(6)$

$-43.5(5)$

$128.4(4)$

$119.5(5)$

$-59.9(5)$

$-13.2(6)$

$167.4(4)$

$-66.3(5)$

$113.4(5)$

$68.6(5)$

-111.7 (4)

$116.0(5)$

$-63.0(5)$

$-19.9(6)$

161.1 (4)

$-65.5(5)$

116.0 (4)

$72.0(5)$

$-106.6(5)$ 
Crystal data

$\left[\mathrm{Fe}\left(\mathrm{C}_{20} \mathrm{H}_{18} \mathrm{~N}_{3}\right)_{2}\left(\mathrm{C}_{4} \mathrm{H}_{8} \mathrm{O}\right)_{2}\right]$

$M_{r}=800.80$

Orthorhombic, $\mathrm{Pbca}$

$a=15.9624(12) \AA$

$b=15.8047(11) \AA$

$c=32.747(2) \AA$

$V=8261.5(10) \AA^{3}$

$Z=8$

$F(000)=3392$

Data collection

Bruker X8 Prospector CCD diffractometer

Radiation source: I-mu-S microsource X-ray tube

Laterally graded multilayer (Goebel) mirror monochromator

$\omega$ and phi scans

Absorption correction: multi-scan

(APEX2; Bruker, 2014)

\section{Refinement}

Refinement on $F^{2}$

Least-squares matrix: full

$R\left[F^{2}>2 \sigma\left(F^{2}\right)\right]=0.043$

$w R\left(F^{2}\right)=0.111$

$S=1.05$

7194 reflections

555 parameters

132 restraints

Primary atom site location: structure-invariant direct methods
$D_{\mathrm{x}}=1.288 \mathrm{Mg} \mathrm{m}^{-3}$

$\mathrm{Cu} K \alpha$ radiation, $\lambda=1.54178 \AA$

Cell parameters from 9933 reflections

$\theta=3.9-66.7^{\circ}$

$\mu=3.29 \mathrm{~mm}^{-1}$

$T=100 \mathrm{~K}$

Block, green

$0.11 \times 0.10 \times 0.06 \mathrm{~mm}$

$T_{\min }=0.569, T_{\max }=0.753$

23792 measured reflections

7194 independent reflections

5411 reflections with $I>2 \sigma(I)$

$R_{\text {int }}=0.055$

$\theta_{\max }=66.7^{\circ}, \theta_{\min }=3.9^{\circ}$

$h=-10 \rightarrow 18$

$k=-18 \rightarrow 18$

$l=-31 \rightarrow 38$

Secondary atom site location: difference Fourier map

Hydrogen site location: inferred from neighbouring sites

$\mathrm{H}$-atom parameters constrained

$w=1 /\left[\sigma^{2}\left(F_{\mathrm{o}}^{2}\right)+(0.0545 P)^{2}+1.2424 P\right]$

where $P=\left(F_{\mathrm{o}}^{2}+2 F_{\mathrm{c}}^{2}\right) / 3$

$(\Delta / \sigma)_{\max }=0.002$

$\Delta \rho_{\max }=0.45$ e $\AA^{-3}$

$\Delta \rho_{\min }=-0.28$ e $\AA^{-3}$

Special details

Geometry. All esds (except the esd in the dihedral angle between two 1.s. planes) are estimated using the full covariance matrix. The cell esds are taken into account individually in the estimation of esds in distances, angles and torsion angles; correlations between esds in cell parameters are only used when they are defined by crystal symmetry. An approximate (isotropic) treatment of cell esds is used for estimating esds involving l.s. planes.

Refinement. One THF ligand is disordered with two alternative orientations. The two moieties were restrained to have similar geometries, and the Uij components of their atoms' ADPs were restrained to be similar if closer than 1.7 Angstrom. Subject to these conditions the occupancy ratio refined to 0.790 (8) to 0.210 (8). Reflection 002 was affected by the beam stop and was omitted from the refinement.

Fractional atomic coordinates and isotropic or equivalent isotropic displacement parameters $\left(\AA^{2}\right)$

\begin{tabular}{llllll}
\hline & $x$ & $y$ & $z$ & $U_{\text {iso }} * / U_{\text {eq }}$ & Occ. $(<1)$ \\
\hline $\mathrm{C} 1$ & $0.01642(16)$ & $0.58940(13)$ & $0.32908(7)$ & $0.0168(5)$ & \\
$\mathrm{C} 2$ & $0.09943(16)$ & $0.58486(14)$ & $0.31399(7)$ & $0.0200(5)$ & \\
$\mathrm{H} 2$ & 0.1417 & 0.5615 & 0.3310 & $0.024^{*}$ &
\end{tabular}




\begin{tabular}{|c|c|c|c|c|}
\hline $\mathrm{C} 3$ & $0.12151(18)$ & $0.61286(14)$ & $0.27560(7)$ & $0.0226(5)$ \\
\hline $\mathrm{H} 3$ & 0.1776 & 0.6063 & 0.2664 & $0.027^{*}$ \\
\hline $\mathrm{C} 4$ & $0.06276(18)$ & $0.65050(15)$ & $0.25038(7)$ & $0.0234(6)$ \\
\hline $\mathrm{H} 4$ & 0.0775 & 0.6699 & 0.2239 & $0.028^{*}$ \\
\hline $\mathrm{C} 5$ & $-0.01885(17)$ & $0.65892(14)$ & $0.26520(7)$ & $0.0208(5)$ \\
\hline H5 & -0.0596 & 0.6861 & 0.2486 & $0.025^{*}$ \\
\hline C6 & $-0.04262(16)$ & $0.62894(13)$ & $0.30332(7)$ & $0.0175(5)$ \\
\hline $\mathrm{C} 7$ & $-0.15464(17)$ & $0.72340(15)$ & $0.31940(7)$ & $0.0210(5)$ \\
\hline $\mathrm{C} 8$ & $-0.21592(18)$ & $0.76162(16)$ & $0.29572(8)$ & $0.0272(6)$ \\
\hline H8 & -0.2452 & 0.7293 & 0.2759 & $0.033^{*}$ \\
\hline C9 & $-0.2349(2)$ & $0.84726(17)$ & $0.30086(9)$ & $0.0355(7)$ \\
\hline H9 & -0.2776 & 0.8730 & 0.2849 & $0.043^{*}$ \\
\hline $\mathrm{C} 10$ & $-0.1909(2)$ & $0.89430(17)$ & $0.32932(9)$ & $0.0378(7)$ \\
\hline H10 & -0.2019 & 0.9531 & 0.3321 & $0.045^{*}$ \\
\hline $\mathrm{C} 11$ & $-0.13083(19)$ & $0.85648(16)$ & $0.35387(8)$ & $0.0305(6)$ \\
\hline H11 & -0.1012 & 0.8895 & 0.3733 & $0.037^{*}$ \\
\hline $\mathrm{C} 12$ & $-0.11369(17)$ & $0.77097(15)$ & $0.35020(7)$ & $0.0216(5)$ \\
\hline C13 & $-0.09703(16)$ & $0.65585(15)$ & $0.39656(7)$ & $0.0191(5)$ \\
\hline $\mathrm{C} 14$ & $-0.16313(17)$ & $0.67221(16)$ & $0.42292(7)$ & $0.0242(6)$ \\
\hline H14 & -0.1838 & 0.7284 & 0.4252 & $0.029^{*}$ \\
\hline $\mathrm{C} 15$ & $-0.19976(17)$ & $0.60877(17)$ & $0.44604(8)$ & $0.0286(6)$ \\
\hline H15 & -0.2445 & 0.6212 & 0.4642 & $0.034 *$ \\
\hline $\mathrm{C} 16$ & $-0.17007(18)$ & $0.52737(17)$ & $0.44225(8)$ & $0.0288(6)$ \\
\hline H16 & -0.1940 & 0.4833 & 0.4582 & $0.035^{*}$ \\
\hline $\mathrm{C} 17$ & -0.10565 (17) & $0.50939(15)$ & $0.41537(8)$ & $0.0247(6)$ \\
\hline H17 & -0.0867 & 0.4526 & 0.4130 & $0.030^{*}$ \\
\hline $\mathrm{C} 18$ & $-0.06699(15)$ & $0.57262(15)$ & $0.39130(7)$ & $0.0181(5)$ \\
\hline C19 & $-0.18646(17)$ & $0.57902(15)$ & $0.29672(8)$ & $0.0265(6)$ \\
\hline H19A & -0.1868 & 0.5873 & 0.2671 & $0.040^{*}$ \\
\hline H19B & -0.2429 & 0.5884 & 0.3075 & $0.040^{*}$ \\
\hline $\mathrm{H} 19 \mathrm{C}$ & -0.1686 & 0.5211 & 0.3030 & $0.040^{*}$ \\
\hline $\mathrm{C} 20$ & $-0.00600(17)$ & $0.77649(15)$ & $0.40344(7)$ & $0.0227(6)$ \\
\hline $\mathrm{H} 20 \mathrm{~A}$ & 0.0224 & 0.8212 & 0.3880 & $0.034^{*}$ \\
\hline H20B & 0.0359 & 0.7399 & 0.4164 & $0.034^{*}$ \\
\hline $\mathrm{H} 20 \mathrm{C}$ & -0.0416 & 0.8020 & 0.4245 & $0.034 *$ \\
\hline $\mathrm{C} 21$ & $0.22608(17)$ & $0.53580(14)$ & $0.42264(7)$ & $0.0206(5)$ \\
\hline $\mathrm{C} 22$ & $0.16839(18)$ & $0.59961(15)$ & $0.43274(8)$ & $0.0264(6)$ \\
\hline $\mathrm{H} 22$ & 0.1155 & 0.6001 & 0.4194 & $0.032 *$ \\
\hline $\mathrm{C} 23$ & $0.18557(19)$ & $0.66182(16)$ & $0.46131(8)$ & $0.0295(6)$ \\
\hline $\mathrm{H} 23$ & 0.1447 & 0.7039 & 0.4671 & $0.035^{*}$ \\
\hline $\mathrm{C} 24$ & $0.26167(19)$ & $0.66321(16)$ & $0.48145(8)$ & $0.0307(6)$ \\
\hline $\mathrm{H} 24$ & 0.2739 & 0.7057 & 0.5011 & $0.037^{*}$ \\
\hline $\mathrm{C} 25$ & $0.31964(18)$ & $0.60072(15)$ & $0.47213(7)$ & $0.0244(6)$ \\
\hline $\mathrm{H} 25$ & 0.3722 & 0.6011 & 0.4857 & $0.029 *$ \\
\hline $\mathrm{C} 26$ & $0.30359(16)$ & $0.53794(14)$ & $0.44382(7)$ & $0.0191(5)$ \\
\hline $\mathrm{C} 27$ & $0.44191(16)$ & $0.50477(14)$ & $0.42074(7)$ & $0.0205(5)$ \\
\hline $\mathrm{C} 28$ & $0.51903(18)$ & $0.49602(16)$ & $0.43994(8)$ & $0.0269(6)$ \\
\hline $\mathrm{H} 28$ & 0.5225 & 0.4653 & 0.4648 & $0.032 *$ \\
\hline
\end{tabular}




\begin{tabular}{|c|c|c|c|c|c|}
\hline $\mathrm{C} 29$ & $0.59142(19)$ & $0.53139(17)$ & $0.42340(9)$ & $0.0333(7)$ & \\
\hline $\mathrm{H} 29$ & 0.6437 & 0.5242 & 0.4368 & $0.040^{*}$ & \\
\hline $\mathrm{C} 30$ & $0.58688(19)$ & $0.57695(18)$ & $0.38751(9)$ & $0.0348(7)$ & \\
\hline $\mathrm{H} 30$ & 0.6354 & 0.6041 & 0.3770 & $0.042 *$ & \\
\hline $\mathrm{C} 31$ & $0.51092(19)$ & $0.58309(15)$ & $0.36667(9)$ & $0.0310(6)$ & \\
\hline H31 & 0.5084 & 0.6136 & 0.3417 & $0.037 *$ & \\
\hline $\mathrm{C} 32$ & $0.43867(17)$ & $0.54505(14)$ & $0.38190(8)$ & $0.0224(5)$ & \\
\hline $\mathrm{C} 33$ & $0.33363(17)$ & $0.45479(15)$ & $0.35471(7)$ & $0.0212(5)$ & \\
\hline C34 & $0.38313(18)$ & 0.40175 (16) & $0.33094(7)$ & $0.0261(6)$ & \\
\hline H34 & 0.4316 & 0.4240 & 0.3180 & $0.031 *$ & \\
\hline $\mathrm{C} 35$ & $0.36331(19)$ & $0.31719(17)$ & $0.32570(8)$ & $0.0312(6)$ & \\
\hline H35 & 0.3968 & 0.2821 & 0.3087 & $0.037^{*}$ & \\
\hline $\mathrm{C} 36$ & $0.29458(19)$ & $0.28494(16)$ & $0.34544(8)$ & $0.0311(6)$ & \\
\hline H36 & 0.2814 & 0.2265 & 0.3430 & $0.037^{*}$ & \\
\hline $\mathrm{C} 37$ & $0.24396(18)$ & $0.33734(15)$ & $0.36908(8)$ & $0.0252(6)$ & \\
\hline H37 & 0.1965 & 0.3139 & 0.3824 & $0.030^{*}$ & \\
\hline C38 & $0.26102(17)$ & $0.42343(15)$ & $0.37376(7)$ & $0.0207(5)$ & \\
\hline C39 & $0.36655(18)$ & $0.40468(15)$ & $0.46536(7)$ & $0.0243(6)$ & \\
\hline H39A & 0.3106 & 0.3792 & 0.4665 & $0.036^{*}$ & \\
\hline H39B & 0.3818 & 0.4259 & 0.4925 & $0.036^{*}$ & \\
\hline $\mathrm{H} 39 \mathrm{C}$ & 0.4074 & 0.3620 & 0.4567 & $0.036^{*}$ & \\
\hline $\mathrm{C} 40$ & $0.3533(2)$ & $0.59413(17)$ & $0.32455(8)$ & $0.0328(7)$ & \\
\hline $\mathrm{H} 40 \mathrm{~A}$ & 0.3929 & 0.5744 & 0.3038 & $0.049 *$ & \\
\hline $\mathrm{H} 40 \mathrm{~B}$ & 0.3655 & 0.6532 & 0.3314 & $0.049^{*}$ & \\
\hline $\mathrm{H} 40 \mathrm{C}$ & 0.2961 & 0.5899 & 0.3139 & $0.049 *$ & \\
\hline C41 & $0.0552(6)$ & $0.3854(4)$ & $0.46578(13)$ & $0.0380(12)$ & $0.790(8)$ \\
\hline $\mathrm{H} 41 \mathrm{~A}$ & 0.0162 & 0.4308 & 0.4744 & $0.046^{*}$ & $0.790(8)$ \\
\hline H41B & 0.1132 & 0.4036 & 0.4719 & $0.046^{*}$ & $0.790(8)$ \\
\hline $\mathrm{C} 42$ & $0.0353(3)$ & $0.3049(4)$ & $0.48744(12)$ & $0.0405(12)$ & $0.790(8)$ \\
\hline $\mathrm{H} 42 \mathrm{~A}$ & 0.0039 & 0.3162 & 0.5129 & $0.049 *$ & $0.790(8)$ \\
\hline $\mathrm{H} 42 \mathrm{~B}$ & 0.0872 & 0.2736 & 0.4942 & $0.049 *$ & $0.790(8)$ \\
\hline $\mathrm{C} 43$ & $-0.0177(3)$ & $0.2556(3)$ & $0.45750(14)$ & $0.0388(11)$ & $0.790(8)$ \\
\hline $\mathrm{H} 43 \mathrm{~A}$ & -0.0122 & 0.1940 & 0.4621 & $0.047 *$ & $0.790(8)$ \\
\hline $\mathrm{H} 43 \mathrm{~B}$ & -0.0775 & 0.2717 & 0.4597 & $0.047^{*}$ & $0.790(8)$ \\
\hline $\mathrm{C} 44$ & $0.0174(4)$ & $0.2806(3)$ & $0.41696(15)$ & $0.0356(12)$ & $0.790(8)$ \\
\hline $\mathrm{H} 44 \mathrm{~A}$ & 0.0648 & 0.2434 & 0.4093 & $0.043 *$ & $0.790(8)$ \\
\hline H44B & -0.0260 & 0.2771 & 0.3954 & $0.043 *$ & $0.790(8)$ \\
\hline C41B & $0.063(2)$ & $0.4049(16)$ & $0.4631(5)$ & $0.038(3)$ & $0.210(8)$ \\
\hline $\mathrm{H} 41 \mathrm{C}$ & 0.0464 & 0.4653 & 0.4641 & $0.046^{*}$ & $0.210(8)$ \\
\hline H41D & 0.1227 & 0.3996 & 0.4703 & $0.046^{*}$ & $0.210(8)$ \\
\hline $\mathrm{C} 42 \mathrm{~B}$ & $0.0085(12)$ & $0.3520(12)$ & $0.4910(4)$ & $0.040(2)$ & $0.210(8)$ \\
\hline $\mathrm{H} 42 \mathrm{C}$ & 0.0338 & 0.3477 & 0.5186 & $0.048^{*}$ & $0.210(8)$ \\
\hline $\mathrm{H} 42 \mathrm{D}$ & -0.0482 & 0.3769 & 0.4936 & $0.048^{*}$ & $0.210(8)$ \\
\hline C43B & $0.0048(15)$ & $0.2675(11)$ & $0.4709(6)$ & $0.040(2)$ & $0.210(8)$ \\
\hline $\mathrm{H} 43 \mathrm{C}$ & -0.0529 & 0.2447 & 0.4721 & $0.048^{*}$ & $0.210(8)$ \\
\hline $\mathrm{H} 43 \mathrm{D}$ & 0.0428 & 0.2273 & 0.4848 & $0.048^{*}$ & $0.210(8)$ \\
\hline C44B & $0.0314(18)$ & $0.2792(9)$ & $0.4274(5)$ & $0.038(3)$ & $0.210(8)$ \\
\hline $\mathrm{H} 44 \mathrm{C}$ & 0.0832 & 0.2468 & 0.4217 & $0.045^{*}$ & $0.210(8)$ \\
\hline
\end{tabular}




\begin{tabular}{|c|c|c|c|c|c|}
\hline H44D & -0.0131 & 0.2596 & 0.4085 & $0.045^{*}$ & $0.210(8)$ \\
\hline $\mathrm{C} 45$ & $0.1079(2)$ & $0.36793(17)$ & $0.29266(7)$ & $0.0310(7)$ & \\
\hline $\mathrm{H} 45 \mathrm{~A}$ & 0.1274 & 0.3086 & 0.2904 & $0.037 *$ & \\
\hline H45B & 0.1574 & 0.4056 & 0.2934 & $0.037^{*}$ & \\
\hline $\mathrm{C} 46$ & $0.0515(2)$ & $0.39048(17)$ & $0.25683(8)$ & $0.0322(7)$ & \\
\hline H46A & 0.0642 & 0.4480 & 0.2466 & $0.039 *$ & \\
\hline H46B & 0.0588 & 0.3496 & 0.2342 & $0.039 *$ & \\
\hline $\mathrm{C} 47$ & $-0.0369(2)$ & $0.3863(2)$ & $0.27371(8)$ & $0.0408(8)$ & \\
\hline H47A & -0.0640 & 0.4425 & 0.2725 & $0.049 *$ & \\
\hline H47B & -0.0711 & 0.3454 & 0.2580 & $0.049 *$ & \\
\hline $\mathrm{C} 48$ & $-0.0273(2)$ & $0.35759(19)$ & $0.31744(8)$ & $0.0374(7)$ & \\
\hline $\mathrm{H} 48 \mathrm{~A}$ & -0.0682 & 0.3869 & 0.3352 & $0.045^{*}$ & \\
\hline H48B & -0.0364 & 0.2958 & 0.3197 & $0.045^{*}$ & \\
\hline N1 & 0.00017 (13) & $0.54988(11)$ & $0.36630(6)$ & $0.0176(4)$ & \\
\hline $\mathrm{N} 2$ & $-0.05762(14)$ & $0.72633(12)$ & $0.37581(6)$ & $0.0194(4)$ & \\
\hline N3 & $-0.12926(13)$ & $0.63808(11)$ & $0.31516(6)$ & $0.0182(4)$ & \\
\hline N4 & $0.20224(13)$ & $0.47367(12)$ & $0.39503(6)$ & $0.0184(4)$ & \\
\hline N5 & $0.36636(14)$ & $0.47409(12)$ & $0.43652(6)$ & $0.0192(4)$ & \\
\hline N6 & $0.36110(14)$ & $0.54189(12)$ & $0.36104(6)$ & $0.0231(5)$ & \\
\hline $\mathrm{Fe} 1$ & $0.08313(2)$ & $0.45812(2)$ & $0.37925(2)$ & $0.01653(11)$ & \\
\hline $\mathrm{O} 1$ & $0.04577(13)$ & $0.36799(10)$ & $0.42238(5)$ & $0.0287(4)$ & \\
\hline $\mathrm{O} 2$ & $0.05724(12)$ & $0.37914(11)$ & $0.32899(5)$ & $0.0272(4)$ & \\
\hline
\end{tabular}

Atomic displacement parameters $\left(\AA^{2}\right)$

\begin{tabular}{|c|c|c|c|c|c|c|}
\hline & $U^{11}$ & $U^{22}$ & $U^{33}$ & $U^{12}$ & $U^{13}$ & $U^{23}$ \\
\hline $\mathrm{C} 1$ & $0.0183(14)$ & $0.0122(10)$ & $0.0199(12)$ & $-0.0029(9)$ & $-0.0020(10)$ & $-0.0015(9)$ \\
\hline $\mathrm{C} 2$ & $0.0200(15)$ & $0.0189(11)$ & $0.0212(12)$ & $0.0012(10)$ & $-0.0024(10)$ & $0.0023(9)$ \\
\hline $\mathrm{C} 3$ & $0.0215(15)$ & $0.0225(11)$ & $0.0238(13)$ & $-0.0024(11)$ & $0.0046(10)$ & $-0.0007(10)$ \\
\hline $\mathrm{C} 4$ & $0.0290(16)$ & $0.0240(12)$ & $0.0172(11)$ & $-0.0043(11)$ & $0.0014(10)$ & $0.0021(10)$ \\
\hline $\mathrm{C} 5$ & $0.0253(15)$ & $0.0167(11)$ & $0.0202(12)$ & $-0.0013(10)$ & $-0.0050(10)$ & $0.0020(9)$ \\
\hline C6 & $0.0187(14)$ & $0.0131(10)$ & 0.0209 (12) & $-0.0021(9)$ & $-0.0004(10)$ & $-0.0033(9)$ \\
\hline $\mathrm{C} 7$ & $0.0200(15)$ & $0.0211(11)$ & $0.0220(12)$ & $0.0013(10)$ & $0.0006(10)$ & $0.0000(10)$ \\
\hline $\mathrm{C} 8$ & $0.0245(16)$ & $0.0287(13)$ & 0.0283 (14) & $0.0004(11)$ & $-0.0067(11)$ & $-0.0022(11)$ \\
\hline C9 & 0.0319 (19) & $0.0312(14)$ & $0.0435(17)$ & $0.0130(13)$ & $-0.0122(13)$ & $0.0004(12)$ \\
\hline $\mathrm{C} 10$ & $0.045(2)$ & $0.0208(12)$ & $0.0480(18)$ & $0.0117(13)$ & $-0.0108(15)$ & $-0.0061(12)$ \\
\hline $\mathrm{C} 11$ & $0.0326(18)$ & $0.0245(13)$ & $0.0344(15)$ & $0.0016(12)$ & $-0.0078(13)$ & $-0.0062(11)$ \\
\hline $\mathrm{C} 12$ & $0.0194(15)$ & $0.0224(12)$ & $0.0230(12)$ & $0.0010(10)$ & $0.0004(10)$ & $-0.0012(10)$ \\
\hline $\mathrm{C} 13$ & $0.0176(14)$ & $0.0238(12)$ & $0.0159(11)$ & $-0.0032(10)$ & -0.0029 (9) & $0.0004(9)$ \\
\hline $\mathrm{C} 14$ & $0.0183(15)$ & $0.0304(13)$ & $0.0238(13)$ & $-0.0014(11)$ & $-0.0021(10)$ & $-0.0058(11)$ \\
\hline $\mathrm{C} 15$ & $0.0180(15)$ & $0.0432(15)$ & $0.0246(13)$ & $-0.0055(12)$ & $0.0062(11)$ & $-0.0049(12)$ \\
\hline $\mathrm{C} 16$ & $0.0231(16)$ & $0.0346(14)$ & $0.0288(14)$ & $-0.0081(12)$ & $0.0028(11)$ & $0.0063(11)$ \\
\hline $\mathrm{C} 17$ & $0.0220(15)$ & $0.0222(12)$ & $0.0299(13)$ & $-0.0017(10)$ & $-0.0001(11)$ & $0.0064(10)$ \\
\hline $\mathrm{C} 18$ & $0.0131(14)$ & $0.0226(11)$ & $0.0185(12)$ & $-0.0010(10)$ & $-0.0027(9)$ & $0.0001(9)$ \\
\hline C19 & $0.0186(15)$ & $0.0253(12)$ & $0.0356(15)$ & $-0.0028(11)$ & $-0.0017(11)$ & $-0.0036(11)$ \\
\hline $\mathrm{C} 20$ & $0.0232(15)$ & $0.0211(12)$ & $0.0237(13)$ & $-0.0024(10)$ & $-0.0027(10)$ & $-0.0006(10)$ \\
\hline $\mathrm{C} 21$ & $0.0224(15)$ & $0.0201(11)$ & $0.0192(12)$ & $0.0013(10)$ & $-0.0010(10)$ & $-0.0013(10)$ \\
\hline $\mathrm{C} 22$ & $0.0200(15)$ & $0.0280(13)$ & $0.0311(14)$ & $0.0033(11)$ & $-0.0092(11)$ & $-0.0051(11)$ \\
\hline
\end{tabular}




\begin{tabular}{|c|c|c|c|c|c|c|}
\hline $\mathrm{C} 23$ & $0.0271(17)$ & $0.0286(13)$ & $0.0330(14)$ & $0.0093(12)$ & $-0.0047(12)$ & $-0.0085(11)$ \\
\hline $\mathrm{C} 24$ & $0.0337(18)$ & $0.0279(13)$ & $0.0307(14)$ & $0.0028(12)$ & $-0.0056(12)$ & $-0.0134(11)$ \\
\hline $\mathrm{C} 25$ & $0.0204(15)$ & $0.0297(13)$ & $0.0230(13)$ & $0.0007(11)$ & $-0.0041(10)$ & $-0.0028(10)$ \\
\hline $\mathrm{C} 26$ & $0.0192(14)$ & $0.0196(11)$ & $0.0184(11)$ & $0.0009(10)$ & $0.0022(9)$ & $-0.0006(9)$ \\
\hline $\mathrm{C} 27$ & $0.0178(14)$ & $0.0192(11)$ & $0.0245(13)$ & $0.0010(10)$ & $0.0041(10)$ & $-0.0058(10)$ \\
\hline $\mathrm{C} 28$ & $0.0255(16)$ & $0.0300(13)$ & $0.0252(13)$ & 0.0030 & $-0.0007(11)$ & $-0.0105(11)$ \\
\hline $\mathrm{C} 29$ & $0.0183(16)$ & $0.0388(15)$ & $0.0428(16)$ & $-0.0010(12)$ & $0.0025(12)$ & $-0.0202(13)$ \\
\hline $\mathrm{C} 30$ & $0.0248(17)$ & $0.0324(14)$ & $0.0473(17)$ & $-0.0103(13)$ & $0.0118(13)$ & $-0.0127(13)$ \\
\hline $\mathrm{C} 31$ & $0.0358(19)$ & $0.0219(12)$ & $0.0351(15)$ & $-0.0038(12)$ & $0.0124(13)$ & $-0.0030(11)$ \\
\hline $\mathrm{C} 32$ & $0.0233(15)$ & 0.0164 (11) & $0.0274(13)$ & $0.0021(10)$ & $0.0073(11)$ & $-0.0048(10)$ \\
\hline $\mathrm{C} 33$ & $0.0271(16)$ & $0.0224(12)$ & $0.0142(11)$ & $0.0056(11)$ & $-0.0040(10)$ & $0.0014(9)$ \\
\hline $\mathrm{C} 34$ & $0.0258(16)$ & $0.0316(13)$ & $0.0208(12)$ & $0.0042(12)$ & $-0.0025(11)$ & $-0.0022(10)$ \\
\hline $\mathrm{C} 35$ & $0.0303(18)$ & $0.0332(14)$ & $0.0301(14)$ & $0.0117(13)$ & $-0.0054(12)$ & $-0.0118(12)$ \\
\hline $\mathrm{C} 36$ & $0.0275(17)$ & $0.0230(13)$ & $0.0430(16)$ & $0.0034(11)$ & $-0.0079(13)$ & $-0.0121(12)$ \\
\hline $\mathrm{C} 37$ & $0.0172(14)$ & $0.0246(13)$ & $0.0339(14)$ & $0.0017(11)$ & $-0.0047(11)$ & $-0.0044(11)$ \\
\hline $\mathrm{C} 38$ & $0.0198(14)$ & $0.0229(12)$ & $0.0195(12)$ & $0.0061(10)$ & $-0.0063(10)$ & $-0.0032(10)$ \\
\hline C39 & $0.0245(16)$ & $0.0252(12)$ & $0.0232(13)$ & $0.0057(11)$ & $0.0027(11)$ & $0.0020(10)$ \\
\hline $\mathrm{C} 40$ & $0.0338(18)$ & $0.0329(14)$ & $0.0316(15)$ & $0.0056(12)$ & $0.0033(12)$ & $0.0116(12)$ \\
\hline $\mathrm{C} 41$ & $0.056(3)$ & $0.038(3)$ & $0.0193(19)$ & $-0.005(2)$ & $-0.0013(19)$ & $0.0042(18)$ \\
\hline $\mathrm{C} 42$ & $0.045(3)$ & $0.047(3)$ & $0.0293(19)$ & $-0.012(2)$ & $-0.0061(17)$ & $0.0139(19)$ \\
\hline $\mathrm{C} 43$ & $0.042(3)$ & $0.044(2)$ & $0.030(2)$ & -0.0157 (19) & $-0.0056(18)$ & $0.0096(18)$ \\
\hline C44 & $0.053(3)$ & $0.0231(17)$ & $0.031(2)$ & $-0.0130(17)$ & $-0.002(2)$ & $0.0099(16)$ \\
\hline $\mathrm{C} 41 \mathrm{~B}$ & $0.048(5)$ & $0.045(5)$ & $0.021(5)$ & $-0.006(5)$ & $0.002(4)$ & $0.016(4)$ \\
\hline $\mathrm{C} 42 \mathrm{~B}$ & $0.047(5)$ & $0.045(5)$ & $0.028(4)$ & $-0.009(4)$ & $-0.003(4)$ & $0.010(4)$ \\
\hline C43B & $0.049(5)$ & $0.040(4)$ & $0.031(5)$ & $-0.009(4)$ & $-0.002(4)$ & $0.013(4)$ \\
\hline C44B & $0.050(5)$ & $0.036(5)$ & $0.028(5)$ & $0.000(4)$ & $-0.005(5)$ & $0.014(4)$ \\
\hline $\mathrm{C} 45$ & $0.041(2)$ & $0.0278(13)$ & $0.0241(13)$ & $0.0064(12)$ & $-0.0021(12)$ & $-0.0067(11)$ \\
\hline $\mathrm{C} 46$ & $0.0403(19)$ & $0.0287(13)$ & $0.0277(14)$ & $0.0020(12)$ & $-0.0069(12)$ & $-0.0015(11)$ \\
\hline $\mathrm{C} 47$ & $0.047(2)$ & $0.0438(16)$ & $0.0322(15)$ & $0.0122(15)$ & $-0.0095(14)$ & $-0.0108(13)$ \\
\hline $\mathrm{C} 48$ & $0.0323(19)$ & $0.0430(16)$ & $0.0369(16)$ & $-0.0113(14)$ & $-0.0083(13)$ & $-0.0070(13)$ \\
\hline N1 & $0.0148(11)$ & $0.0189(9)$ & $0.0189(9)$ & $0.0010(8)$ & $-0.0010(8)$ & $0.0041(8)$ \\
\hline $\mathrm{N} 2$ & $0.0216(12)$ & $0.0185(9)$ & $0.0180(10)$ & $-0.0009(8)$ & $-0.0027(8)$ & $-0.0007(8)$ \\
\hline N3 & $0.0170(12)$ & $0.0162(9)$ & $0.0215(10)$ & $-0.0005(8)$ & $-0.0035(8)$ & $-0.0012(8)$ \\
\hline N4 & $0.0136(12)$ & $0.0175(9)$ & $0.0242(10)$ & $0.0030(8)$ & $-0.0037(8)$ & $-0.0020(8)$ \\
\hline N5 & $0.0173(12)$ & $0.0210(9)$ & $0.0193(10)$ & $0.0014(8)$ & $-0.0009(8)$ & $0.0003(8)$ \\
\hline N6 & $0.0270(13)$ & $0.0207(10)$ & $0.0217(10)$ & $0.0014(9)$ & $0.0017(9)$ & $0.0020(8)$ \\
\hline $\mathrm{Fe} 1$ & $0.0167(2)$ & $0.01553(17)$ & 0.01733 (19) & $0.00079(15)$ & $-0.00282(15)$ & $0.00016(15)$ \\
\hline $\mathrm{O} 1$ & $0.0407(13)$ & $0.0233(8)$ & $0.0220(9)$ & $-0.0009(8)$ & $0.0016(8)$ & $0.0036(7)$ \\
\hline $\mathrm{O} 2$ & $0.0302(12)$ & $0.0271(9)$ & $0.0242(9)$ & $-0.0020(8)$ & $-0.0053(8)$ & $-0.0073(7)$ \\
\hline
\end{tabular}

Geometric parameters ( $\left.\AA,{ }^{\circ}\right)$

\begin{tabular}{llll}
\hline $\mathrm{C} 1-\mathrm{N} 1$ & $1.394(3)$ & $\mathrm{C} 31-\mathrm{H} 31$ & 0.9500 \\
$\mathrm{C} 1-\mathrm{C} 6$ & $1.411(3)$ & $\mathrm{C} 32-\mathrm{N} 6$ & $1.415(4)$ \\
$\mathrm{C} 1-\mathrm{C} 2$ & $1.416(4)$ & $\mathrm{C} 33-\mathrm{C} 34$ & $1.390(4)$ \\
$\mathrm{C} 2-\mathrm{C} 3$ & $1.378(3)$ & $\mathrm{C} 33-\mathrm{C} 38$ & $1.406(4)$ \\
$\mathrm{C} 2-\mathrm{H} 2$ & 0.9500 & $\mathrm{C} 33-\mathrm{N} 6$ & $1.460(3)$ \\
$\mathrm{C} 3-\mathrm{C} 4$ & $1.384(4)$ & $\mathrm{C} 34-\mathrm{C} 35$ & $1.384(4)$
\end{tabular}




\begin{tabular}{|c|c|c|c|}
\hline $\mathrm{C} 3-\mathrm{H} 3$ & 0.9500 & C34-H34 & 0.9500 \\
\hline $\mathrm{C} 4-\mathrm{C} 5$ & $1.397(4)$ & $\mathrm{C} 35-\mathrm{C} 36$ & $1.372(4)$ \\
\hline $\mathrm{C} 4-\mathrm{H} 4$ & 0.9500 & $\mathrm{C} 35-\mathrm{H} 35$ & 0.9500 \\
\hline $\mathrm{C} 5-\mathrm{C} 6$ & $1.388(3)$ & C36-C37 & $1.392(4)$ \\
\hline $\mathrm{C} 5-\mathrm{H} 5$ & 0.9500 & $\mathrm{C} 36-\mathrm{H} 36$ & 0.9500 \\
\hline $\mathrm{C} 6-\mathrm{N} 3$ & $1.444(3)$ & $\mathrm{C} 37-\mathrm{C} 38$ & $1.396(3)$ \\
\hline $\mathrm{C} 7-\mathrm{C} 8$ & $1.387(4)$ & C37-H37 & 0.9500 \\
\hline $\mathrm{C} 7-\mathrm{N} 3$ & 1.415 & $\mathrm{C} 38-\mathrm{N} 4$ & $1.413(3)$ \\
\hline $\mathrm{C} 7-\mathrm{C} 12$ & $1.418(3)$ & $\mathrm{C} 39-\mathrm{N} 5$ & $1.448(3)$ \\
\hline $\mathrm{C} 8-\mathrm{C} 9$ & $1.397(4)$ & C39-H39A & 0.9800 \\
\hline $\mathrm{C} 8-\mathrm{H} 8$ & 0.9500 & $\mathrm{C} 39-\mathrm{H} 39 \mathrm{~B}$ & 0.9800 \\
\hline $\mathrm{C} 9-\mathrm{C} 10$ & $1.384(4)$ & $\mathrm{C} 39-\mathrm{H} 39 \mathrm{C}$ & 0.9800 \\
\hline $\mathrm{C} 9-\mathrm{H} 9$ & 0.9500 & $\mathrm{C} 40-\mathrm{N} 6$ & $1.458(3)$ \\
\hline $\mathrm{C} 10-\mathrm{C} 11$ & $1.386(4)$ & $\mathrm{C} 40-\mathrm{H} 40 \mathrm{~A}$ & 0.9800 \\
\hline $\mathrm{C} 10-\mathrm{H} 10$ & 0.9500 & $\mathrm{C} 40-\mathrm{H} 40 \mathrm{~B}$ & 0.9800 \\
\hline $\mathrm{C} 11-\mathrm{C} 12$ & $1.384(4)$ & $\mathrm{C} 40-\mathrm{H} 40 \mathrm{C}$ & 0.9800 \\
\hline $\mathrm{C} 11-\mathrm{H} 11$ & 0.9500 & $\mathrm{C} 41-\mathrm{O} 1$ & $1.455(5)$ \\
\hline $\mathrm{C} 12-\mathrm{N} 2$ & $1.415(3)$ & $\mathrm{C} 41-\mathrm{C} 42$ & $1.490(6)$ \\
\hline $\mathrm{C} 13-\mathrm{C} 14$ & $1.388(4)$ & $\mathrm{C} 41-\mathrm{H} 41 \mathrm{~A}$ & 0.9900 \\
\hline $\mathrm{C} 13-\mathrm{C} 18$ & $1.411(3)$ & $\mathrm{C} 41-\mathrm{H} 41 \mathrm{~B}$ & 0.9900 \\
\hline $\mathrm{C} 13-\mathrm{N} 2$ & $1.448(3)$ & $\mathrm{C} 42-\mathrm{C} 43$ & $1.512(6)$ \\
\hline $\mathrm{C} 14-\mathrm{C} 15$ & $1.386(4)$ & $\mathrm{C} 42-\mathrm{H} 42 \mathrm{~A}$ & 0.9900 \\
\hline C14-H14 & 0.9500 & $\mathrm{C} 42-\mathrm{H} 42 \mathrm{~B}$ & 0.9900 \\
\hline $\mathrm{C} 15-\mathrm{C} 16$ & $1.377(4)$ & $\mathrm{C} 43-\mathrm{C} 44$ & $1.495(5)$ \\
\hline $\mathrm{C} 15-\mathrm{H} 15$ & 0.9500 & $\mathrm{C} 43-\mathrm{H} 43 \mathrm{~A}$ & 0.9900 \\
\hline $\mathrm{C} 16-\mathrm{C} 17$ & $1.383(4)$ & $\mathrm{C} 43-\mathrm{H} 43 \mathrm{~B}$ & 0.9900 \\
\hline C16-H16 & 0.9500 & $\mathrm{C} 44-\mathrm{O} 1$ & $1.464(4)$ \\
\hline $\mathrm{C} 17-\mathrm{C} 18$ & $1.414(3)$ & $\mathrm{C} 44-\mathrm{H} 44 \mathrm{~A}$ & 0.9900 \\
\hline $\mathrm{C} 17-\mathrm{H} 17$ & 0.9500 & $\mathrm{C} 44-\mathrm{H} 44 \mathrm{~B}$ & 0.9900 \\
\hline $\mathrm{C} 18-\mathrm{N} 1$ & $1.396(3)$ & $\mathrm{C} 41 \mathrm{~B}-\mathrm{O} 1$ & $1.479(14)$ \\
\hline $\mathrm{C} 19-\mathrm{N} 3$ & 1.439 & $\mathrm{C} 41 \mathrm{~B}-\mathrm{C} 42 \mathrm{~B}$ & $1.512(17)$ \\
\hline C19-H19A & 0.9800 & $\mathrm{C} 41 \mathrm{~B}-\mathrm{H} 41 \mathrm{C}$ & 0.9900 \\
\hline C19-H19B & 0.9800 & $\mathrm{C} 41 \mathrm{~B}-\mathrm{H} 41 \mathrm{D}$ & 0.9900 \\
\hline C19-H19C & 0.9800 & $\mathrm{C} 42 \mathrm{~B}-\mathrm{C} 43 \mathrm{~B}$ & $1.492(16)$ \\
\hline $\mathrm{C} 20-\mathrm{N} 2$ & $1.458(3)$ & $\mathrm{C} 42 \mathrm{~B}-\mathrm{H} 42 \mathrm{C}$ & 0.9900 \\
\hline $\mathrm{C} 20-\mathrm{H} 20 \mathrm{~A}$ & 0.9800 & $\mathrm{C} 42 \mathrm{~B}-\mathrm{H} 42 \mathrm{D}$ & 0.9900 \\
\hline $\mathrm{C} 20-\mathrm{H} 20 \mathrm{~B}$ & 0.9800 & $\mathrm{C} 43 \mathrm{~B}-\mathrm{C} 44 \mathrm{~B}$ & $1.497(15)$ \\
\hline $\mathrm{C} 20-\mathrm{H} 20 \mathrm{C}$ & 0.9800 & $\mathrm{C} 43 \mathrm{~B}-\mathrm{H} 43 \mathrm{C}$ & 0.9900 \\
\hline $\mathrm{C} 21-\mathrm{N} 4$ & $1.388(3)$ & $\mathrm{C} 43 \mathrm{~B}-\mathrm{H} 43 \mathrm{D}$ & 0.9900 \\
\hline $\mathrm{C} 21-\mathrm{C} 22$ & $1.405(4)$ & $\mathrm{C} 44 \mathrm{~B}-\mathrm{O} 1$ & $1.431(14)$ \\
\hline $\mathrm{C} 21-\mathrm{C} 26$ & $1.419(4)$ & $\mathrm{C} 44 \mathrm{~B}-\mathrm{H} 44 \mathrm{C}$ & 0.9900 \\
\hline $\mathrm{C} 22-\mathrm{C} 23$ & $1.385(3)$ & $\mathrm{C} 44 \mathrm{~B}-\mathrm{H} 44 \mathrm{D}$ & 0.9900 \\
\hline $\mathrm{C} 22-\mathrm{H} 22$ & 0.9500 & $\mathrm{C} 45-\mathrm{O} 2$ & $1.449(3)$ \\
\hline $\mathrm{C} 23-\mathrm{C} 24$ & $1.382(4)$ & $\mathrm{C} 45-\mathrm{C} 46$ & $1.522(4)$ \\
\hline $\mathrm{C} 23-\mathrm{H} 23$ & 0.9500 & $\mathrm{C} 45-\mathrm{H} 45 \mathrm{~A}$ & 0.9900 \\
\hline $\mathrm{C} 24-\mathrm{C} 25$ & $1.387(4)$ & $\mathrm{C} 45-\mathrm{H} 45 \mathrm{~B}$ & 0.9900 \\
\hline $\mathrm{C} 24-\mathrm{H} 24$ & 0.9500 & $\mathrm{C} 46-\mathrm{C} 47$ & $1.516(5)$ \\
\hline $\mathrm{C} 25-\mathrm{C} 26$ & $1.382(3)$ & $\mathrm{C} 46-\mathrm{H} 46 \mathrm{~A}$ & 0.9900 \\
\hline
\end{tabular}




\begin{tabular}{|c|c|c|c|}
\hline $\mathrm{C} 25-\mathrm{H} 25$ & 0.9500 & $\mathrm{C} 46-\mathrm{H} 46 \mathrm{~B}$ & 0.9900 \\
\hline $\mathrm{C} 26-\mathrm{N} 5$ & $1.442(3)$ & $\mathrm{C} 47-\mathrm{C} 48$ & $1.510(4)$ \\
\hline $\mathrm{C} 27-\mathrm{C} 28$ & $1.389(4)$ & $\mathrm{C} 47-\mathrm{H} 47 \mathrm{~A}$ & 0.9900 \\
\hline $\mathrm{C} 27-\mathrm{N} 5$ & $1.399(3)$ & $\mathrm{C} 47-\mathrm{H} 47 \mathrm{~B}$ & 0.9900 \\
\hline $\mathrm{C} 27-\mathrm{C} 32$ & $1.423(4)$ & $\mathrm{C} 48-\mathrm{O} 2$ & $1.442(3)$ \\
\hline $\mathrm{C} 28-\mathrm{C} 29$ & $1.393(4)$ & $\mathrm{C} 48-\mathrm{H} 48 \mathrm{~A}$ & 0.9900 \\
\hline $\mathrm{C} 28-\mathrm{H} 28$ & 0.9500 & $\mathrm{C} 48-\mathrm{H} 48 \mathrm{~B}$ & 0.9900 \\
\hline $\mathrm{C} 29-\mathrm{C} 30$ & $1.380(4)$ & $\mathrm{N} 1-\mathrm{Fe} 1$ & $2.0092(19)$ \\
\hline C29- & 0.9500 & $\mathrm{~N} 4-\mathrm{Fe} 1$ & $1.985(2)$ \\
\hline $\mathrm{C} 30-\mathrm{C} 31$ & $1.395(4)$ & $\mathrm{Fe} 1-\mathrm{O} 1$ & $2.0926(17)$ \\
\hline $\mathrm{C} 30-\mathrm{H} 30$ & 0.9500 & $\mathrm{Fe} 1-\mathrm{O} 2$ & $2.1068(16)$ \\
\hline $\mathrm{C} 31-\mathrm{C} 32$ & $1.393(4)$ & & \\
\hline $\mathrm{N} 1-\mathrm{C} 1-\mathrm{C} 6$ & $126.7(2)$ & $\mathrm{C} 33-\mathrm{C} 38-\mathrm{N} 4$ & $124.6(2)$ \\
\hline $\mathrm{N} 1-\mathrm{C} 1-\mathrm{C} 2$ & $117.2(2)$ & $\mathrm{N} 5-\mathrm{C} 39-\mathrm{H} 39 \mathrm{~A}$ & 109.5 \\
\hline $\mathrm{C} 6-\mathrm{C} 1-\mathrm{C} 2$ & $116.0(2)$ & N5-C39-H39B & 109.5 \\
\hline $\mathrm{C} 3-\mathrm{C} 2-\mathrm{C} 1$ & $122.8(2)$ & $\mathrm{H} 39 \mathrm{~A}-\mathrm{C} 39-\mathrm{H} 39 \mathrm{~B}$ & 109.5 \\
\hline $\mathrm{C} 3-\mathrm{C} 2-\mathrm{H} 2$ & 118.6 & $\mathrm{~N} 5-\mathrm{C} 39-\mathrm{H} 39 \mathrm{C}$ & 109.5 \\
\hline $\mathrm{C} 1-\mathrm{C} 2-\mathrm{H} 2$ & 118.6 & $\mathrm{H} 39 \mathrm{~A}-\mathrm{C} 39-\mathrm{H} 39 \mathrm{C}$ & 109.5 \\
\hline $\mathrm{C} 2-\mathrm{C} 3-\mathrm{C} 4$ & $120.6(3)$ & $\mathrm{H} 39 \mathrm{~B}-\mathrm{C} 39-\mathrm{H} 39 \mathrm{C}$ & 109.5 \\
\hline $\mathrm{C} 2-\mathrm{C} 3-\mathrm{H} 3$ & 119.7 & $\mathrm{~N} 6-\mathrm{C} 40-\mathrm{H} 40 \mathrm{~A}$ & 109.5 \\
\hline $\mathrm{C} 4-\mathrm{C} 3-\mathrm{H} 3$ & 119.7 & $\mathrm{~N} 6-\mathrm{C} 40-\mathrm{H} 40 \mathrm{~B}$ & 109.5 \\
\hline $\mathrm{C} 3-\mathrm{C} 4-\mathrm{C} 5$ & $117.7(2)$ & $\mathrm{H} 40 \mathrm{~A}-\mathrm{C} 40-\mathrm{H} 40 \mathrm{~B}$ & 109.5 \\
\hline $\mathrm{C} 3-\mathrm{C} 4-\mathrm{H} 4$ & 121.1 & $\mathrm{~N} 6-\mathrm{C} 40-\mathrm{H} 40 \mathrm{C}$ & 109.5 \\
\hline $\mathrm{C} 5-\mathrm{C} 4-\mathrm{H} 4$ & 121.1 & $\mathrm{H} 40 \mathrm{~A}-\mathrm{C} 40-\mathrm{H} 40 \mathrm{C}$ & 109.5 \\
\hline $\mathrm{C} 6-\mathrm{C} 5-\mathrm{C} 4$ & $122.3(2)$ & $\mathrm{H} 40 \mathrm{~B}-\mathrm{C} 40-\mathrm{H} 40 \mathrm{C}$ & 109.5 \\
\hline $\mathrm{C} 6-\mathrm{C} 5-\mathrm{H} 5$ & 118.8 & $\mathrm{O} 1-\mathrm{C} 41-\mathrm{C} 42$ & $106.3(3)$ \\
\hline $\mathrm{C} 4-\mathrm{C} 5-\mathrm{H} 5$ & 118.8 & $\mathrm{O} 1-\mathrm{C} 41-\mathrm{H} 41 \mathrm{~A}$ & 110.5 \\
\hline $\mathrm{C} 5-\mathrm{C} 6-\mathrm{C} 1$ & $120.4(2)$ & $\mathrm{C} 42-\mathrm{C} 41-\mathrm{H} 41 \mathrm{~A}$ & 110.5 \\
\hline $\mathrm{C} 5-\mathrm{C} 6-\mathrm{N} 3$ & $118.0(2)$ & $\mathrm{O} 1-\mathrm{C} 41-\mathrm{H} 41 \mathrm{~B}$ & 110.5 \\
\hline $\mathrm{C} 1-\mathrm{C} 6-\mathrm{N} 3$ & $121.6(2)$ & $\mathrm{C} 42-\mathrm{C} 41-\mathrm{H} 41 \mathrm{~B}$ & 110.5 \\
\hline $\mathrm{C} 8-\mathrm{C} 7-\mathrm{N} 3$ & $124.2(2)$ & $\mathrm{H} 41 \mathrm{~A}-\mathrm{C} 41-\mathrm{H} 41 \mathrm{~B}$ & 108.7 \\
\hline $\mathrm{C} 8-\mathrm{C} 7-\mathrm{C} 12$ & $119.5(2)$ & $\mathrm{C} 41-\mathrm{C} 42-\mathrm{C} 43$ & $104.5(4)$ \\
\hline $\mathrm{N} 3-\mathrm{C} 7-\mathrm{C} 12$ & $116.3(2)$ & $\mathrm{C} 41-\mathrm{C} 42-\mathrm{H} 42 \mathrm{~A}$ & 110.9 \\
\hline $\mathrm{C} 7-\mathrm{C} 8-\mathrm{C} 9$ & 120.5 & $\mathrm{C} 43-\mathrm{C} 42-\mathrm{H} 42 \mathrm{~A}$ & 110.9 \\
\hline $\mathrm{C} 7-\mathrm{C} 8-\mathrm{H} 8$ & 119.7 & $\mathrm{C} 41-\mathrm{C} 42-\mathrm{H} 42 \mathrm{~B}$ & 110.9 \\
\hline $\mathrm{C} 9-\mathrm{C} 8-\mathrm{H} 8$ & 119.7 & $\mathrm{C} 43-\mathrm{C} 42-\mathrm{H} 42 \mathrm{~B}$ & 110.9 \\
\hline $\mathrm{C} 10-\mathrm{C} 9-\mathrm{C} 8$ & $119.4(3)$ & $\mathrm{H} 42 \mathrm{~A}-\mathrm{C} 42-\mathrm{H} 42 \mathrm{~B}$ & 108.9 \\
\hline $\mathrm{C} 10-\mathrm{C} 9-\mathrm{H} 9$ & 120.3 & $\mathrm{C} 44-\mathrm{C} 43-\mathrm{C} 42$ & 103.3 \\
\hline $\mathrm{C} 8-\mathrm{C} 9-\mathrm{H} 9$ & 120.3 & $\mathrm{C} 44-\mathrm{C} 43-\mathrm{H} 43 \mathrm{~A}$ & 111.1 \\
\hline $\mathrm{C} 9-\mathrm{C} 10-\mathrm{C} 11$ & $120.7(2)$ & $\mathrm{C} 42-\mathrm{C} 43-\mathrm{H} 43 \mathrm{~A}$ & 111.1 \\
\hline $\mathrm{C} 9-\mathrm{C} 10-\mathrm{H} 10$ & 119.7 & $\mathrm{C} 44-\mathrm{C} 43-\mathrm{H} 43 \mathrm{~B}$ & 111.1 \\
\hline $\mathrm{C} 11-\mathrm{C} 10-\mathrm{H} 10$ & 119.7 & $\mathrm{C} 42-\mathrm{C} 43-\mathrm{H} 43 \mathrm{~B}$ & 111.1 \\
\hline $\mathrm{C} 12-\mathrm{C} 11-\mathrm{C} 10$ & $120.5(3)$ & $\mathrm{H} 43 \mathrm{~A}-\mathrm{C} 43-\mathrm{H} 43 \mathrm{~B}$ & 109.1 \\
\hline $\mathrm{C} 12-\mathrm{C} 11-\mathrm{H} 11$ & 119.8 & $\mathrm{O} 1-\mathrm{C} 44-\mathrm{C} 43$ & $104.9(3)$ \\
\hline $\mathrm{C} 10-\mathrm{C} 11-\mathrm{H} 11$ & 119.8 & $\mathrm{O} 1-\mathrm{C} 44-\mathrm{H} 44 \mathrm{~A}$ & 110.8 \\
\hline $\mathrm{C} 11-\mathrm{C} 12-\mathrm{N} 2$ & $124.1(2)$ & $\mathrm{C} 43-\mathrm{C} 44-\mathrm{H} 44 \mathrm{~A}$ & 110.8 \\
\hline $\mathrm{C} 11-\mathrm{C} 12-\mathrm{C} 7$ & $119.2(2)$ & $\mathrm{O} 1-\mathrm{C} 44-\mathrm{H} 44 \mathrm{~B}$ & 110.8 \\
\hline
\end{tabular}




\begin{tabular}{|c|c|c|c|}
\hline $\mathrm{N} 2-\mathrm{C} 12-\mathrm{C} 7$ & $116.7(2)$ & $\mathrm{C} 43-\mathrm{C} 44-\mathrm{H} 44 \mathrm{~B}$ & 110.8 \\
\hline $\mathrm{C} 14-\mathrm{C} 13-\mathrm{C} 18$ & $120.5(2)$ & $\mathrm{H} 44 \mathrm{~A}-\mathrm{C} 44-\mathrm{H} 44 \mathrm{~B}$ & 108.8 \\
\hline $\mathrm{C} 14-\mathrm{C} 13-\mathrm{N} 2$ & $118.6(2)$ & $\mathrm{O} 1-\mathrm{C} 41 \mathrm{~B}-\mathrm{C} 42 \mathrm{~B}$ & $102.9(12)$ \\
\hline $\mathrm{C} 18-\mathrm{C} 13-\mathrm{N} 2$ & $120.8(2)$ & $\mathrm{O} 1-\mathrm{C} 41 \mathrm{~B}-\mathrm{H} 41 \mathrm{C}$ & 111.2 \\
\hline $\mathrm{C} 15-\mathrm{C} 14-\mathrm{C} 13$ & $121.7(2)$ & $\mathrm{C} 42 \mathrm{~B}-\mathrm{C} 41 \mathrm{~B}-\mathrm{H} 41 \mathrm{C}$ & 111.2 \\
\hline $\mathrm{C} 15-\mathrm{C} 14-\mathrm{H} 14$ & 119.1 & $\mathrm{O} 1-\mathrm{C} 41 \mathrm{~B}-\mathrm{H} 41 \mathrm{D}$ & 111.2 \\
\hline $\mathrm{C} 13-\mathrm{C} 14-\mathrm{H} 14$ & 119.1 & $\mathrm{C} 42 \mathrm{~B}-\mathrm{C} 41 \mathrm{~B}-\mathrm{H} 41 \mathrm{D}$ & 111.2 \\
\hline $\mathrm{C} 16-\mathrm{C} 15-\mathrm{C} 14$ & $118.8(2)$ & $\mathrm{H} 41 \mathrm{C}-\mathrm{C} 41 \mathrm{~B}-\mathrm{H} 41 \mathrm{D}$ & 109.1 \\
\hline $\mathrm{C} 16-\mathrm{C} 15-\mathrm{H} 15$ & 120.6 & $\mathrm{C} 43 \mathrm{~B}-\mathrm{C} 42 \mathrm{~B}-\mathrm{C} 41 \mathrm{~B}$ & $104.4(13)$ \\
\hline $\mathrm{C} 14-\mathrm{C} 15-\mathrm{H} 15$ & 120.6 & $\mathrm{C} 43 \mathrm{~B}-\mathrm{C} 42 \mathrm{~B}-\mathrm{H} 42 \mathrm{C}$ & 110.9 \\
\hline $\mathrm{C} 15-\mathrm{C} 16-\mathrm{C} 17$ & $120.4(2)$ & $\mathrm{C} 41 \mathrm{~B}-\mathrm{C} 42 \mathrm{~B}-\mathrm{H} 42 \mathrm{C}$ & 110.9 \\
\hline $\mathrm{C} 15-\mathrm{C} 16-\mathrm{H} 16$ & 119.8 & $\mathrm{C} 43 \mathrm{~B}-\mathrm{C} 42 \mathrm{~B}-\mathrm{H} 42 \mathrm{D}$ & 110.9 \\
\hline $\mathrm{C} 17-\mathrm{C} 16-\mathrm{H} 16$ & 119.8 & $\mathrm{C} 41 \mathrm{~B}-\mathrm{C} 42 \mathrm{~B}-\mathrm{H} 42 \mathrm{D}$ & 110.9 \\
\hline $\mathrm{C} 16-\mathrm{C} 17-\mathrm{C} 18$ & $122.3(2)$ & $\mathrm{H} 42 \mathrm{C}-\mathrm{C} 42 \mathrm{~B}-\mathrm{H} 42 \mathrm{D}$ & 108.9 \\
\hline $\mathrm{C} 16-\mathrm{C} 17-\mathrm{H} 17$ & 118.9 & $\mathrm{C} 42 \mathrm{~B}-\mathrm{C} 43 \mathrm{~B}-\mathrm{C} 44 \mathrm{~B}$ & $107.4(12)$ \\
\hline $\mathrm{C} 18-\mathrm{C} 17-\mathrm{H} 17$ & 118.9 & $\mathrm{C} 42 \mathrm{~B}-\mathrm{C} 43 \mathrm{~B}-\mathrm{H} 43 \mathrm{C}$ & 110.2 \\
\hline $\mathrm{N} 1-\mathrm{C} 18-\mathrm{C} 13$ & $124.9(2)$ & $\mathrm{C} 44 \mathrm{~B}-\mathrm{C} 43 \mathrm{~B}-\mathrm{H} 43 \mathrm{C}$ & 110.2 \\
\hline $\mathrm{N} 1-\mathrm{C} 18-\mathrm{C} 17$ & $118.7(2)$ & $\mathrm{C} 42 \mathrm{~B}-\mathrm{C} 43 \mathrm{~B}-\mathrm{H} 43 \mathrm{D}$ & 110.2 \\
\hline $\mathrm{C} 13-\mathrm{C} 18-\mathrm{C} 17$ & $116.3(2)$ & $\mathrm{C} 44 \mathrm{~B}-\mathrm{C} 43 \mathrm{~B}-\mathrm{H} 43 \mathrm{D}$ & 110.2 \\
\hline N3-C19-H19A & 109.5 & $\mathrm{H} 43 \mathrm{C}-\mathrm{C} 43 \mathrm{~B}-\mathrm{H} 43 \mathrm{D}$ & 108.5 \\
\hline N3-C19-H19B & 109.5 & $\mathrm{O} 1-\mathrm{C} 44 \mathrm{~B}-\mathrm{C} 43 \mathrm{~B}$ & $106.0(12)$ \\
\hline $\mathrm{H} 19 \mathrm{~A}-\mathrm{C} 19-\mathrm{H} 19 \mathrm{~B}$ & 109.5 & $\mathrm{O} 1-\mathrm{C} 44 \mathrm{~B}-\mathrm{H} 44 \mathrm{C}$ & 110.5 \\
\hline $\mathrm{N} 3-\mathrm{C} 19-\mathrm{H} 19 \mathrm{C}$ & 109.5 & $\mathrm{C} 43 \mathrm{~B}-\mathrm{C} 44 \mathrm{~B}-\mathrm{H} 44 \mathrm{C}$ & 110.5 \\
\hline $\mathrm{H} 19 \mathrm{~A}-\mathrm{C} 19-\mathrm{H} 19 \mathrm{C}$ & 109.5 & $\mathrm{O} 1-\mathrm{C} 44 \mathrm{~B}-\mathrm{H} 44 \mathrm{D}$ & 110.5 \\
\hline $\mathrm{H} 19 \mathrm{~B}-\mathrm{C} 19-\mathrm{H} 19 \mathrm{C}$ & 109.5 & $\mathrm{C} 43 \mathrm{~B}-\mathrm{C} 44 \mathrm{~B}-\mathrm{H} 44 \mathrm{D}$ & 110.5 \\
\hline $\mathrm{N} 2-\mathrm{C} 20-\mathrm{H} 20 \mathrm{~A}$ & 109.5 & $\mathrm{H} 44 \mathrm{C}-\mathrm{C} 44 \mathrm{~B}-\mathrm{H} 44 \mathrm{D}$ & 108.7 \\
\hline $\mathrm{N} 2-\mathrm{C} 20-\mathrm{H} 20 \mathrm{~B}$ & 109.5 & $\mathrm{O} 2-\mathrm{C} 45-\mathrm{C} 46$ & $105.9(2)$ \\
\hline $\mathrm{H} 20 \mathrm{~A}-\mathrm{C} 20-\mathrm{H} 20 \mathrm{~B}$ & 109.5 & $\mathrm{O} 2-\mathrm{C} 45-\mathrm{H} 45 \mathrm{~A}$ & 110.6 \\
\hline $\mathrm{N} 2-\mathrm{C} 20-\mathrm{H} 20 \mathrm{C}$ & 109.5 & $\mathrm{C} 46-\mathrm{C} 45-\mathrm{H} 45 \mathrm{~A}$ & 110.6 \\
\hline $\mathrm{H} 20 \mathrm{~A}-\mathrm{C} 20-\mathrm{H} 20 \mathrm{C}$ & 109.5 & $\mathrm{O} 2-\mathrm{C} 45-\mathrm{H} 45 \mathrm{~B}$ & 110.6 \\
\hline $\mathrm{H} 20 \mathrm{~B}-\mathrm{C} 20-\mathrm{H} 20 \mathrm{C}$ & 109.5 & $\mathrm{C} 46-\mathrm{C} 45-\mathrm{H} 45 \mathrm{~B}$ & 110.6 \\
\hline $\mathrm{N} 4-\mathrm{C} 21-\mathrm{C} 22$ & $118.8(2)$ & $\mathrm{H} 45 \mathrm{~A}-\mathrm{C} 45-\mathrm{H} 45 \mathrm{~B}$ & 108.7 \\
\hline $\mathrm{N} 4-\mathrm{C} 21-\mathrm{C} 26$ & $125.1(2)$ & $\mathrm{C} 47-\mathrm{C} 46-\mathrm{C} 45$ & $105.0(2)$ \\
\hline $\mathrm{C} 22-\mathrm{C} 21-\mathrm{C} 26$ & $116.1(2)$ & $\mathrm{C} 47-\mathrm{C} 46-\mathrm{H} 46 \mathrm{~A}$ & 110.7 \\
\hline $\mathrm{C} 23-\mathrm{C} 22-\mathrm{C} 21$ & $122.6(3)$ & $\mathrm{C} 45-\mathrm{C} 46-\mathrm{H} 46 \mathrm{~A}$ & 110.7 \\
\hline $\mathrm{C} 23-\mathrm{C} 22-\mathrm{H} 22$ & 118.7 & $\mathrm{C} 47-\mathrm{C} 46-\mathrm{H} 46 \mathrm{~B}$ & 110.7 \\
\hline $\mathrm{C} 21-\mathrm{C} 22-\mathrm{H} 22$ & 118.7 & $\mathrm{C} 45-\mathrm{C} 46-\mathrm{H} 46 \mathrm{~B}$ & 110.7 \\
\hline $\mathrm{C} 24-\mathrm{C} 23-\mathrm{C} 22$ & $120.5(2)$ & $\mathrm{H} 46 \mathrm{~A}-\mathrm{C} 46-\mathrm{H} 46 \mathrm{~B}$ & 108.8 \\
\hline $\mathrm{C} 24-\mathrm{C} 23-\mathrm{H} 23$ & 119.7 & $\mathrm{C} 48-\mathrm{C} 47-\mathrm{C} 46$ & $105.4(3)$ \\
\hline $\mathrm{C} 22-\mathrm{C} 23-\mathrm{H} 23$ & 119.7 & $\mathrm{C} 48-\mathrm{C} 47-\mathrm{H} 47 \mathrm{~A}$ & 110.7 \\
\hline $\mathrm{C} 23-\mathrm{C} 24-\mathrm{C} 25$ & $118.0(2)$ & $\mathrm{C} 46-\mathrm{C} 47-\mathrm{H} 47 \mathrm{~A}$ & 110.7 \\
\hline $\mathrm{C} 23-\mathrm{C} 24-\mathrm{H} 24$ & 121.0 & $\mathrm{C} 48-\mathrm{C} 47-\mathrm{H} 47 \mathrm{~B}$ & 110.7 \\
\hline $\mathrm{C} 25-\mathrm{C} 24-\mathrm{H} 24$ & 121.0 & $\mathrm{C} 46-\mathrm{C} 47-\mathrm{H} 47 \mathrm{~B}$ & 110.7 \\
\hline $\mathrm{C} 26-\mathrm{C} 25-\mathrm{C} 24$ & $122.4(3)$ & $\mathrm{H} 47 \mathrm{~A}-\mathrm{C} 47-\mathrm{H} 47 \mathrm{~B}$ & 108.8 \\
\hline $\mathrm{C} 26-\mathrm{C} 25-\mathrm{H} 25$ & 118.8 & $\mathrm{O} 2-\mathrm{C} 48-\mathrm{C} 47$ & $105.8(2)$ \\
\hline $\mathrm{C} 24-\mathrm{C} 25-\mathrm{H} 25$ & 118.8 & $\mathrm{O} 2-\mathrm{C} 48-\mathrm{H} 48 \mathrm{~A}$ & 110.6 \\
\hline $\mathrm{C} 25-\mathrm{C} 26-\mathrm{C} 21$ & $120.5(2)$ & $\mathrm{C} 47-\mathrm{C} 48-\mathrm{H} 48 \mathrm{~A}$ & 110.6 \\
\hline $\mathrm{C} 25-\mathrm{C} 26-\mathrm{N} 5$ & $119.0(2)$ & $\mathrm{O} 2-\mathrm{C} 48-\mathrm{H} 48 \mathrm{~B}$ & 110.6 \\
\hline
\end{tabular}




\begin{tabular}{|c|c|c|c|}
\hline $\mathrm{C} 21-\mathrm{C} 26-\mathrm{N} 5$ & $120.5(2)$ & $\mathrm{C} 47-\mathrm{C} 48-\mathrm{H} 48 \mathrm{~B}$ & 110.6 \\
\hline $\mathrm{C} 28-\mathrm{C} 27-\mathrm{N} 5$ & $124.2(2)$ & $\mathrm{H} 48 \mathrm{~A}-\mathrm{C} 48-\mathrm{H} 48 \mathrm{~B}$ & 108.7 \\
\hline $\mathrm{C} 28-\mathrm{C} 27-\mathrm{C} 32$ & $118.8(2)$ & $\mathrm{C} 1-\mathrm{N} 1-\mathrm{C} 18$ & $122.71(19)$ \\
\hline $\mathrm{N} 5-\mathrm{C} 27-\mathrm{C} 32$ & $117.0(2)$ & $\mathrm{C} 1-\mathrm{N} 1-\mathrm{Fe} 1$ & $112.67(16)$ \\
\hline $\mathrm{C} 27-\mathrm{C} 28-\mathrm{C} 29$ & $121.3(3)$ & $\mathrm{C} 18-\mathrm{N} 1-\mathrm{Fe} 1$ & $124.62(15)$ \\
\hline $\mathrm{C} 27-\mathrm{C} 28-\mathrm{H} 28$ & 119.4 & $\mathrm{C} 12-\mathrm{N} 2-\mathrm{C} 13$ & $112.8(2)$ \\
\hline $\mathrm{C} 29-\mathrm{C} 28-\mathrm{H} 28$ & 119.4 & $\mathrm{C} 12-\mathrm{N} 2-\mathrm{C} 20$ & $117.01(19)$ \\
\hline $\mathrm{C} 30-\mathrm{C} 29-\mathrm{C} 28$ & $119.8(3)$ & $\mathrm{C} 13-\mathrm{N} 2-\mathrm{C} 20$ & $111.86(17)$ \\
\hline $\mathrm{C} 30-\mathrm{C} 29-\mathrm{H} 29$ & 120.1 & $\mathrm{C} 7-\mathrm{N} 3-\mathrm{C} 19$ & $118.6(2)$ \\
\hline $\mathrm{C} 28-\mathrm{C} 29-\mathrm{H} 29$ & 120.1 & $\mathrm{C} 7-\mathrm{N} 3-\mathrm{C} 6$ & $113.33(19)$ \\
\hline $\mathrm{C} 29-\mathrm{C} 30-\mathrm{C} 31$ & $119.9(3)$ & $\mathrm{C} 19-\mathrm{N} 3-\mathrm{C} 6$ & $115.48(19)$ \\
\hline $\mathrm{C} 29-\mathrm{C} 30-\mathrm{H} 30$ & 120.0 & $\mathrm{C} 21-\mathrm{N} 4-\mathrm{C} 38$ & $122.4(2)$ \\
\hline $\mathrm{C} 31-\mathrm{C} 30-\mathrm{H} 30$ & 120.0 & $\mathrm{C} 21-\mathrm{N} 4-\mathrm{Fe} 1$ & $121.30(16)$ \\
\hline $\mathrm{C} 32-\mathrm{C} 31-\mathrm{C} 30$ & $121.0(3)$ & $\mathrm{C} 38-\mathrm{N} 4-\mathrm{Fe} 1$ & $115.99(15)$ \\
\hline $\mathrm{C} 32-\mathrm{C} 31-\mathrm{H} 31$ & 119.5 & $\mathrm{C} 27-\mathrm{N} 5-\mathrm{C} 26$ & $114.68(19)$ \\
\hline $\mathrm{C} 30-\mathrm{C} 31-\mathrm{H} 31$ & 119.5 & $\mathrm{C} 27-\mathrm{N} 5-\mathrm{C} 39$ & $120.1(2)$ \\
\hline $\mathrm{C} 31-\mathrm{C} 32-\mathrm{N} 6$ & $124.5(2)$ & $\mathrm{C} 26-\mathrm{N} 5-\mathrm{C} 39$ & $115.07(19)$ \\
\hline $\mathrm{C} 31-\mathrm{C} 32-\mathrm{C} 27$ & $118.9(3)$ & $\mathrm{C} 32-\mathrm{N} 6-\mathrm{C} 40$ & $116.8(2)$ \\
\hline $\mathrm{N} 6-\mathrm{C} 32-\mathrm{C} 27$ & $116.6(2)$ & $\mathrm{C} 32-\mathrm{N} 6-\mathrm{C} 33$ & $111.40(19)$ \\
\hline $\mathrm{C} 34-\mathrm{C} 33-\mathrm{C} 38$ & $120.3(2)$ & $\mathrm{C} 40-\mathrm{N} 6-\mathrm{C} 33$ & $113.1(2)$ \\
\hline $\mathrm{C} 34-\mathrm{C} 33-\mathrm{N} 6$ & $118.5(2)$ & $\mathrm{N} 4-\mathrm{Fe} 1-\mathrm{N} 1$ & $126.64(8)$ \\
\hline $\mathrm{C} 38-\mathrm{C} 33-\mathrm{N} 6$ & $121.1(2)$ & $\mathrm{N} 4-\mathrm{Fe} 1-\mathrm{O} 1$ & $100.46(8)$ \\
\hline $\mathrm{C} 35-\mathrm{C} 34-\mathrm{C} 33$ & $121.5(3)$ & $\mathrm{N} 1-\mathrm{Fe} 1-\mathrm{O} 1$ & $116.49(8)$ \\
\hline C $35-\mathrm{C} 34-\mathrm{H} 34$ & 119.3 & $\mathrm{~N} 4-\mathrm{Fe} 1-\mathrm{O} 2$ & $117.67(8)$ \\
\hline $\mathrm{C} 33-\mathrm{C} 34-\mathrm{H} 34$ & 119.3 & $\mathrm{~N} 1-\mathrm{Fe} 1-\mathrm{O} 2$ & $97.67(8)$ \\
\hline $\mathrm{C} 36-\mathrm{C} 35-\mathrm{C} 34$ & $118.9(3)$ & $\mathrm{O} 1-\mathrm{Fe} 1-\mathrm{O} 2$ & $93.90(7)$ \\
\hline $\mathrm{C} 36-\mathrm{C} 35-\mathrm{H} 35$ & 120.6 & $\mathrm{C} 41-\mathrm{O} 1-\mathrm{C} 44$ & $109.2(3)$ \\
\hline C34-C35-H35 & 120.6 & $\mathrm{C} 44 \mathrm{~B}-\mathrm{O} 1-\mathrm{C} 41 \mathrm{~B}$ & $108.2(11)$ \\
\hline $\mathrm{C} 35-\mathrm{C} 36-\mathrm{C} 37$ & $120.3(2)$ & $\mathrm{C} 44 \mathrm{~B}-\mathrm{O} 1-\mathrm{Fe} 1$ & $142.2(8)$ \\
\hline $\mathrm{C} 35-\mathrm{C} 36-\mathrm{H} 36$ & 119.8 & $\mathrm{C} 41-\mathrm{O} 1-\mathrm{Fe} 1$ & $120.1(2)$ \\
\hline $\mathrm{C} 37-\mathrm{C} 36-\mathrm{H} 36$ & 119.8 & $\mathrm{C} 44-\mathrm{O} 1-\mathrm{Fe} 1$ & $130.4(2)$ \\
\hline $\mathrm{C} 36-\mathrm{C} 37-\mathrm{C} 38$ & $121.8(3)$ & $\mathrm{C} 41 \mathrm{~B}-\mathrm{O} 1-\mathrm{Fe} 1$ & $106.7(7)$ \\
\hline $\mathrm{C} 36-\mathrm{C} 37-\mathrm{H} 37$ & 119.1 & $\mathrm{C} 48-\mathrm{O} 2-\mathrm{C} 45$ & $106.1(2)$ \\
\hline $\mathrm{C} 38-\mathrm{C} 37-\mathrm{H} 37$ & 119.1 & $\mathrm{C} 48-\mathrm{O} 2-\mathrm{Fe} 1$ & $121.89(17)$ \\
\hline $\mathrm{C} 37-\mathrm{C} 38-\mathrm{C} 33$ & $117.1(2)$ & $\mathrm{C} 45-\mathrm{O} 2-\mathrm{Fe} 1$ & $127.18(16)$ \\
\hline $\mathrm{C} 37-\mathrm{C} 38-\mathrm{N} 4$ & $118.2(2)$ & & \\
\hline $\mathrm{N} 1-\mathrm{C} 1-\mathrm{C} 2-\mathrm{C} 3$ & $172.5(2)$ & $\mathrm{C} 42-\mathrm{C} 43-\mathrm{C} 44-\mathrm{O} 1$ & $-32.1(5)$ \\
\hline $\mathrm{C} 6-\mathrm{C} 1-\mathrm{C} 2-\mathrm{C} 3$ & $-3.5(3)$ & $\mathrm{O} 1-\mathrm{C} 41 \mathrm{~B}-\mathrm{C} 42 \mathrm{~B}-\mathrm{C} 43 \mathrm{~B}$ & $30(3)$ \\
\hline $\mathrm{C} 1-\mathrm{C} 2-\mathrm{C} 3-\mathrm{C} 4$ & $2.7(4)$ & $\mathrm{C} 41 \mathrm{~B}-\mathrm{C} 42 \mathrm{~B}-\mathrm{C} 43 \mathrm{~B}-\mathrm{C} 44 \mathrm{~B}$ & $-18(3)$ \\
\hline $\mathrm{C} 2-\mathrm{C} 3-\mathrm{C} 4-\mathrm{C} 5$ & $0.0(3)$ & $\mathrm{C} 42 \mathrm{~B}-\mathrm{C} 43 \mathrm{~B}-\mathrm{C} 44 \mathrm{~B}-\mathrm{O} 1$ & $-3(2)$ \\
\hline $\mathrm{C} 3-\mathrm{C} 4-\mathrm{C} 5-\mathrm{C} 6$ & $-1.9(3)$ & $\mathrm{O} 2-\mathrm{C} 45-\mathrm{C} 46-\mathrm{C} 47$ & $-17.9(3)$ \\
\hline $\mathrm{C} 4-\mathrm{C} 5-\mathrm{C} 6-\mathrm{C} 1$ & $1.0(3)$ & $\mathrm{C} 45-\mathrm{C} 46-\mathrm{C} 47-\mathrm{C} 48$ & $-2.7(3)$ \\
\hline $\mathrm{C} 4-\mathrm{C} 5-\mathrm{C} 6-\mathrm{N} 3$ & $-177.8(2)$ & $\mathrm{C} 46-\mathrm{C} 47-\mathrm{C} 48-\mathrm{O} 2$ & $22.5(3)$ \\
\hline $\mathrm{N} 1-\mathrm{C} 1-\mathrm{C} 6-\mathrm{C} 5$ & $-173.9(2)$ & $\mathrm{C} 6-\mathrm{C} 1-\mathrm{N} 1-\mathrm{C} 18$ & $-27.5(3)$ \\
\hline $\mathrm{C} 2-\mathrm{C} 1-\mathrm{C} 6-\mathrm{C} 5$ & $1.6(3)$ & $\mathrm{C} 2-\mathrm{C} 1-\mathrm{N} 1-\mathrm{C} 18$ & $157.1(2)$ \\
\hline $\mathrm{N} 1-\mathrm{C} 1-\mathrm{C} 6-\mathrm{N} 3$ & $4.8(3)$ & $\mathrm{C} 6-\mathrm{C} 1-\mathrm{N} 1-\mathrm{Fe} 1$ & $152.43(18)$ \\
\hline $\mathrm{C} 2-\mathrm{C} 1-\mathrm{C} 6-\mathrm{N} 3$ & $-179.66(19)$ & $\mathrm{C} 2-\mathrm{C} 1-\mathrm{N} 1-\mathrm{Fe} 1$ & $-23.0(2)$ \\
\hline
\end{tabular}




\begin{tabular}{|c|c|}
\hline $\mathrm{N} 3-\mathrm{C} 7-\mathrm{C} 8-\mathrm{C} 9$ & $-177.5(3)$ \\
\hline $\mathrm{C} 12-\mathrm{C} 7-\mathrm{C} 8-\mathrm{C} 9$ & $2.7(4)$ \\
\hline $\mathrm{C} 7-\mathrm{C} 8-\mathrm{C} 9-\mathrm{C} 10$ & $1.2(5)$ \\
\hline $\mathrm{C} 8-\mathrm{C} 9-\mathrm{C} 10-\mathrm{C} 11$ & $-2.7(5)$ \\
\hline $\mathrm{C} 9-\mathrm{C} 10-\mathrm{C} 11-\mathrm{C} 12$ & $0.2(5)$ \\
\hline $\mathrm{C} 10-\mathrm{C} 11-\mathrm{C} 12-\mathrm{N} 2$ & $-175.6(3)$ \\
\hline $\mathrm{C} 10-\mathrm{C} 11-\mathrm{C} 12-\mathrm{C} 7$ & $3.7(4)$ \\
\hline $\mathrm{C} 8-\mathrm{C} 7-\mathrm{C} 12-\mathrm{C} 11$ & $-5.2(4)$ \\
\hline $\mathrm{N} 3-\mathrm{C} 7-\mathrm{C} 12-\mathrm{C} 11$ & $175.0(2)$ \\
\hline $\mathrm{C} 8-\mathrm{C} 7-\mathrm{C} 12-\mathrm{N} 2$ & $174.2(2)$ \\
\hline $\mathrm{N} 3-\mathrm{C} 7-\mathrm{C} 12-\mathrm{N} 2$ & $-5.6(3)$ \\
\hline $\mathrm{C} 18-\mathrm{C} 13-\mathrm{C} 14-\mathrm{C} 15$ & $2.5(4)$ \\
\hline $\mathrm{N} 2-\mathrm{C} 13-\mathrm{C} 14-\mathrm{C} 15$ & $-175.2(2)$ \\
\hline $\mathrm{C} 13-\mathrm{C} 14-\mathrm{C} 15-\mathrm{C} 16$ & $-0.7(4)$ \\
\hline $\mathrm{C} 14-\mathrm{C} 15-\mathrm{C} 16-\mathrm{C} 17$ & $-0.9(4)$ \\
\hline $\mathrm{C} 15-\mathrm{C} 16-\mathrm{C} 17-\mathrm{C} 18$ & $0.9(4)$ \\
\hline $\mathrm{C} 14-\mathrm{C} 13-\mathrm{C} 18-\mathrm{N} 1$ & $-178.5(2)$ \\
\hline $\mathrm{N} 2-\mathrm{C} 13-\mathrm{C} 18-\mathrm{N} 1$ & $-0.8(4)$ \\
\hline $\mathrm{C} 14-\mathrm{C} 13-\mathrm{C} 18-\mathrm{C} 17$ & $-2.4(3)$ \\
\hline $\mathrm{N} 2-\mathrm{C} 13-\mathrm{C} 18-\mathrm{C} 17$ & $175.2(2)$ \\
\hline $\mathrm{C} 16-\mathrm{C} 17-\mathrm{C} 18-\mathrm{N} 1$ & $177.1(2)$ \\
\hline $\mathrm{C} 16-\mathrm{C} 17-\mathrm{C} 18-\mathrm{C} 13$ & $0.8(4)$ \\
\hline $\mathrm{N} 4-\mathrm{C} 21-\mathrm{C} 22-\mathrm{C} 23$ & $177.1(2)$ \\
\hline $\mathrm{C} 26-\mathrm{C} 21-\mathrm{C} 22-\mathrm{C} 23$ & $0.6(4)$ \\
\hline $\mathrm{C} 21-\mathrm{C} 22-\mathrm{C} 23-\mathrm{C} 24$ & $-0.2(4)$ \\
\hline $\mathrm{C} 22-\mathrm{C} 23-\mathrm{C} 24-\mathrm{C} 25$ & $0.0(4)$ \\
\hline $\mathrm{C} 23-\mathrm{C} 24-\mathrm{C} 25-\mathrm{C} 26$ & $-0.2(4)$ \\
\hline $\mathrm{C} 24-\mathrm{C} 25-\mathrm{C} 26-\mathrm{C} 21$ & $0.6(4)$ \\
\hline $\mathrm{C} 24-\mathrm{C} 25-\mathrm{C} 26-\mathrm{N} 5$ & $-178.6(2)$ \\
\hline $\mathrm{N} 4-\mathrm{C} 21-\mathrm{C} 26-\mathrm{C} 25$ & $-177.0(2)$ \\
\hline $\mathrm{C} 22-\mathrm{C} 21-\mathrm{C} 26-\mathrm{C} 25$ & $-0.8(3)$ \\
\hline $\mathrm{N} 4-\mathrm{C} 21-\mathrm{C} 26-\mathrm{N} 5$ & $2.2(4)$ \\
\hline $\mathrm{C} 22-\mathrm{C} 21-\mathrm{C} 26-\mathrm{N} 5$ & $178.4(2)$ \\
\hline $\mathrm{N} 5-\mathrm{C} 27-\mathrm{C} 28-\mathrm{C} 29$ & $-177.1(2)$ \\
\hline $\mathrm{C} 32-\mathrm{C} 27-\mathrm{C} 28-\mathrm{C} 29$ & $4.9(3)$ \\
\hline $\mathrm{C} 27-\mathrm{C} 28-\mathrm{C} 29-\mathrm{C} 30$ & $0.8(4)$ \\
\hline $\mathrm{C} 28-\mathrm{C} 29-\mathrm{C} 30-\mathrm{C} 31$ & $-3.9(4)$ \\
\hline $\mathrm{C} 29-\mathrm{C} 30-\mathrm{C} 31-\mathrm{C} 32$ & $1.2(4)$ \\
\hline $\mathrm{C} 30-\mathrm{C} 31-\mathrm{C} 32-\mathrm{N} 6$ & $-174.2(2)$ \\
\hline $\mathrm{C} 30-\mathrm{C} 31-\mathrm{C} 32-\mathrm{C} 27$ & $4.5(4)$ \\
\hline $\mathrm{C} 28-\mathrm{C} 27-\mathrm{C} 32-\mathrm{C} 31$ & $-7.5(3)$ \\
\hline $\mathrm{N} 5-\mathrm{C} 27-\mathrm{C} 32-\mathrm{C} 31$ & $174.4(2)$ \\
\hline $\mathrm{C} 28-\mathrm{C} 27-\mathrm{C} 32-\mathrm{N} 6$ & $171.4(2)$ \\
\hline $\mathrm{N} 5-\mathrm{C} 27-\mathrm{C} 32-\mathrm{N} 6$ & $-6.7(3)$ \\
\hline $\mathrm{C} 38-\mathrm{C} 33-\mathrm{C} 34-\mathrm{C} 35$ & $-0.7(4)$ \\
\hline $\mathrm{N} 6-\mathrm{C} 33-\mathrm{C} 34-\mathrm{C} 35$ & $176.1(2)$ \\
\hline $\mathrm{C} 33-\mathrm{C} 34-\mathrm{C} 35-\mathrm{C} 36$ & $-1.9(4)$ \\
\hline $\mathrm{C} 34-\mathrm{C} 35-\mathrm{C} 36-\mathrm{C} 37$ & $2.4(4)$ \\
\hline
\end{tabular}

\begin{tabular}{|c|c|}
\hline $\mathrm{C} 13-\mathrm{C} 18-\mathrm{N} 1-\mathrm{C} 1$ & $-36.1(3)$ \\
\hline $\mathrm{C} 17-\mathrm{C} 18-\mathrm{N} 1-\mathrm{C} 1$ & $147.9(2)$ \\
\hline $\mathrm{C} 13-\mathrm{C} 18-\mathrm{N} 1-\mathrm{Fe} 1$ & $144.0(2)$ \\
\hline $\mathrm{C} 17-\mathrm{C} 18-\mathrm{N} 1-\mathrm{Fe} 1$ & $-31.9(3)$ \\
\hline $\mathrm{C} 11-\mathrm{C} 12-\mathrm{N} 2-\mathrm{C} 13$ & $122.2(3)$ \\
\hline $\mathrm{C} 7-\mathrm{C} 12-\mathrm{N} 2-\mathrm{C} 13$ & $-57.1(3)$ \\
\hline $\mathrm{C} 11-\mathrm{C} 12-\mathrm{N} 2-\mathrm{C} 20$ & $-9.6(4)$ \\
\hline $\mathrm{C} 7-\mathrm{C} 12-\mathrm{N} 2-\mathrm{C} 20$ & $171.1(2)$ \\
\hline $\mathrm{C} 14-\mathrm{C} 13-\mathrm{N} 2-\mathrm{C} 12$ & $-61.2(3)$ \\
\hline $\mathrm{C} 18-\mathrm{C} 13-\mathrm{N} 2-\mathrm{C} 12$ & $121.1(2)$ \\
\hline $\mathrm{C} 14-\mathrm{C} 13-\mathrm{N} 2-\mathrm{C} 20$ & $73.2(3)$ \\
\hline $\mathrm{C} 18-\mathrm{C} 13-\mathrm{N} 2-\mathrm{C} 20$ & $-104.5(3)$ \\
\hline $\mathrm{C} 8-\mathrm{C} 7-\mathrm{N} 3-\mathrm{C} 19$ & $-23.0(4)$ \\
\hline $\mathrm{C} 12-\mathrm{C} 7-\mathrm{N} 3-\mathrm{C} 19$ & $156.8(2)$ \\
\hline $\mathrm{C} 8-\mathrm{C} 7-\mathrm{N} 3-\mathrm{C} 6$ & $117.2(3)$ \\
\hline $\mathrm{C} 12-\mathrm{C} 7-\mathrm{N} 3-\mathrm{C} 6$ & $-63.0(3)$ \\
\hline $\mathrm{C} 5-\mathrm{C} 6-\mathrm{N} 3-\mathrm{C} 7$ & $-64.9(3)$ \\
\hline $\mathrm{C} 1-\mathrm{C} 6-\mathrm{N} 3-\mathrm{C} 7$ & $116.4(2)$ \\
\hline $\mathrm{C} 5-\mathrm{C} 6-\mathrm{N} 3-\mathrm{C} 19$ & $76.6(3)$ \\
\hline $\mathrm{C} 1-\mathrm{C} 6-\mathrm{N} 3-\mathrm{C} 19$ & $-102.2(2)$ \\
\hline $\mathrm{C} 22-\mathrm{C} 21-\mathrm{N} 4-\mathrm{C} 38$ & $160.3(2)$ \\
\hline $\mathrm{C} 26-\mathrm{C} 21-\mathrm{N} 4-\mathrm{C} 38$ & $-23.6(4)$ \\
\hline $\mathrm{C} 22-\mathrm{C} 21-\mathrm{N} 4-\mathrm{Fe} 1$ & $-13.7(3)$ \\
\hline $\mathrm{C} 26-\mathrm{C} 21-\mathrm{N} 4-\mathrm{Fe} 1$ & $162.43(19)$ \\
\hline $\mathrm{C} 37-\mathrm{C} 38-\mathrm{N} 4-\mathrm{C} 21$ & $139.0(2)$ \\
\hline $\mathrm{C} 33-\mathrm{C} 38-\mathrm{N} 4-\mathrm{C} 21$ & $-45.0(3)$ \\
\hline $\mathrm{C} 37-\mathrm{C} 38-\mathrm{N} 4-\mathrm{Fe} 1$ & $-46.7(3)$ \\
\hline $\mathrm{C} 33-\mathrm{C} 38-\mathrm{N} 4-\mathrm{Fe} 1$ & $129.3(2)$ \\
\hline $\mathrm{C} 28-\mathrm{C} 27-\mathrm{N} 5-\mathrm{C} 26$ & $120.0(2)$ \\
\hline $\mathrm{C} 32-\mathrm{C} 27-\mathrm{N} 5-\mathrm{C} 26$ & $-62.0(3)$ \\
\hline $\mathrm{C} 28-\mathrm{C} 27-\mathrm{N} 5-\mathrm{C} 39$ & $-23.7(3)$ \\
\hline $\mathrm{C} 32-\mathrm{C} 27-\mathrm{N} 5-\mathrm{C} 39$ & $154.3(2)$ \\
\hline $\mathrm{C} 25-\mathrm{C} 26-\mathrm{N} 5-\mathrm{C} 27$ & $-62.8(3)$ \\
\hline $\mathrm{C} 21-\mathrm{C} 26-\mathrm{N} 5-\mathrm{C} 27$ & $118.0(2)$ \\
\hline $\mathrm{C} 25-\mathrm{C} 26-\mathrm{N} 5-\mathrm{C} 39$ & $82.8(3)$ \\
\hline $\mathrm{C} 21-\mathrm{C} 26-\mathrm{N} 5-\mathrm{C} 39$ & $-96.4(3)$ \\
\hline $\mathrm{C} 31-\mathrm{C} 32-\mathrm{N} 6-\mathrm{C} 40$ & $-10.8(3)$ \\
\hline $\mathrm{C} 27-\mathrm{C} 32-\mathrm{N} 6-\mathrm{C} 40$ & $170.5(2)$ \\
\hline $\mathrm{C} 31-\mathrm{C} 32-\mathrm{N} 6-\mathrm{C} 33$ & $121.3(2)$ \\
\hline $\mathrm{C} 27-\mathrm{C} 32-\mathrm{N} 6-\mathrm{C} 33$ & $-57.5(3)$ \\
\hline $\mathrm{C} 34-\mathrm{C} 33-\mathrm{N} 6-\mathrm{C} 32$ & $-62.3(3)$ \\
\hline $\mathrm{C} 38-\mathrm{C} 33-\mathrm{N} 6-\mathrm{C} 32$ & $114.5(3)$ \\
\hline $\mathrm{C} 34-\mathrm{C} 33-\mathrm{N} 6-\mathrm{C} 40$ & $71.6(3)$ \\
\hline $\mathrm{C} 38-\mathrm{C} 33-\mathrm{N} 6-\mathrm{C} 40$ & $-111.6(3)$ \\
\hline $\mathrm{C} 43 \mathrm{~B}-\mathrm{C} 44 \mathrm{~B}-\mathrm{O} 1-\mathrm{C} 41 \mathrm{~B}$ & $23(3)$ \\
\hline $\mathrm{C} 43 \mathrm{~B}-\mathrm{C} 44 \mathrm{~B}-\mathrm{O} 1-\mathrm{Fe} 1$ & $179.4(10)$ \\
\hline $\mathrm{C} 42-\mathrm{C} 41-\mathrm{O} 1-\mathrm{C} 44$ & $2.4(8)$ \\
\hline $\mathrm{C} 42-\mathrm{C} 41-\mathrm{O} 1-\mathrm{Fe} 1$ & $-171.7(4)$ \\
\hline
\end{tabular}


supporting information

$\begin{array}{llll}\mathrm{C} 35-\mathrm{C} 36-\mathrm{C} 37-\mathrm{C} 38 & -0.3(4) & \mathrm{C} 43-\mathrm{C} 44-\mathrm{O} 1-\mathrm{C} 41 & 18.9(6) \\ \mathrm{C} 36-\mathrm{C} 37-\mathrm{C} 38-\mathrm{C} 33 & -2.3(4) & \mathrm{C} 43-\mathrm{C} 44-\mathrm{O} 1-\mathrm{Fe} 1 & -167.9(2) \\ \mathrm{C} 36-\mathrm{C} 37-\mathrm{C} 38-\mathrm{N} 4 & 174.1(2) & \mathrm{C} 42 \mathrm{~B}-\mathrm{C} 41 \mathrm{~B}-\mathrm{O} 1-\mathrm{C} 44 \mathrm{~B} & -34(3) \\ \mathrm{C} 34-\mathrm{C} 33-\mathrm{C} 38-\mathrm{C} 37 & 2.7(4) & \mathrm{C} 42 \mathrm{~B}-\mathrm{C} 41 \mathrm{~B}-\mathrm{O} 1-\mathrm{Fe} 1 & 161.2(16) \\ \mathrm{N} 6-\mathrm{C} 33-\mathrm{C} 38-\mathrm{C} 37 & -174.0(2) & \mathrm{C} 47-\mathrm{C} 48-\mathrm{O} 2-\mathrm{C} 45 & -34.6(3) \\ \mathrm{C} 34-\mathrm{C} 33-\mathrm{C} 38-\mathrm{N} 4 & -173.3(2) & \mathrm{C} 47-\mathrm{C} 48-\mathrm{O} 2-\mathrm{Fe} 1 & 122.5(2) \\ \mathrm{N} 6-\mathrm{C} 33-\mathrm{C} 38-\mathrm{N} 4 & 9.9(4) & \mathrm{C} 46-\mathrm{C} 45-\mathrm{O} 2-\mathrm{C} 48 & 32.7(3) \\ \mathrm{O} 1-\mathrm{C} 41-\mathrm{C} 42-\mathrm{C} 43 & -22.4(7) & \mathrm{C} 46-\mathrm{C} 45-\mathrm{O} 2-\mathrm{Fe} 1 & -122.7(2) \\ \mathrm{C} 41-\mathrm{C} 42-\mathrm{C} 43-\mathrm{C} 44 & 33.5(6) & & \end{array}$

